\title{
Lectures on Matroids
}

\author{
W. T. Tutte \\ Professor of Mathematics, University of Waterloo, Ontario, Canada
}

(November 16, 1964)

This work is based on a series of papers in the Transactions of the American Mathematical Society: A homotopy theorem for matroids, I and II, 88, 144-174 (1958); and Matroids and graphs, 90, $572-552$ (1959).

These papers set out a theory of matroids, with special emphasis on the conditions for a matroid to represent a graph. The treatment is as rigorous as that in the original papers, but it is hoped that the present work is easier to read, because less condensed. Many theorems originally proved only for "regular matroids" have been generalized to the less restricted class of "binary" ones, and the last part of the work has been improved by the incorporation of a theory of "even" matroids.

\section{Contents}

1. Matroids

1.1. Matroids.....

1.2. Chain-groups

2. Duality.

2.1. Principal forests

2.2. Dendroids.

2.3. Dendroids of a chain-group

2.4. Dual chain-groups.

2.5. Bond-matroid of a graph.

2.6. Dual matroids

3. Structure of matroids

3.1. Subgraphs and contractions

3.2. Minors of chain-groups

3.3. Minors of matroids

3.4. Connection in matroids

3.5. Properties of rank

4. Geometry of matroids

4.1. Flats

4.2. Connected flats

4.3. Linear subclasses

4.4. Carriers......

5. Specializations

5.1. Matroids and chain-groups

5.2. Representative matrices....

5.3. Characterization of binary matroids
5.4. Regular chain-groups and matroids

\section{Matroids}

\subsection{Matroids}

Given a graph $G$ we denote the set of vertices by $V(G)$ and the set of edges by $E(G)$.

In general $G$ contains a number of polygons, and each polygon can be specified by its set of edges. Suppose a complete list is made of all the polygons of $G$, each as a set of edges. Then this list can be described as a class of non-null subsets of $E(G)$. We denote it by $P(G)$, and, anticipating a later definition, we call it the polygon-matroid of $G$.
5.5. Some binary matroids which are not regular........ 18

5.6. Graphic and cographic matroids....................... 19

6. Homotopy ........................................... 20

6.1. Elementary re-entrant paths.......................... 20

6.2. Homotopy $\ldots \ldots \ldots \ldots \ldots \ldots \ldots \ldots \ldots \ldots \ldots \ldots \ldots \ldots \ldots, 21$

6.3. A lemma......................................... 21

6.4. Homotopy theorem................................ 25

6.5. Special cases........................................... 27

7. Characterization of regular matroids...................... 27

7.1. Some preliminary observations........................ 27

7.2. The Fano configuration............................. 27

7.3. Heptahedron ......................................... 28

7.4. Condition for a regular matroid...................... 29

7.5. Characterization of regular matroid................... 32

8. The matroid and the atom............................... 32

8.1. General matroid.................................... 32

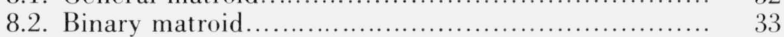

8.3. Kuratowski graphs.................................... 34

8.4. Skewness............................................. 34

8.5. Segments determined by bridges......................... 35

8.6. Regular matroid................................. 37

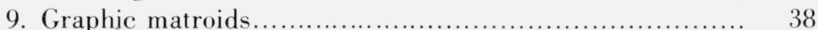

9.1. Bonds .......................................... 38

9.2. Bridges in a bond-matroid.............................. 40

9.3. Property of odd matroids............................. 42

9.4. Characterization of graphic matroids..................... 46

The definition of $P(G)$ immediately suggests a difficult graph-theoretical problem. Suppose we are given a class $M$ of non-null subsets of a fixed set $E$. How can we determine whether or not $M$ can be interpreted as the polygon-matroid of a graph? A partial answer can be given at once; it is not difficult to show that the two following conditions are necessary.

I No member of $M$ is a proper subset of another.

II Let $a$ and $b$ be two members of $E$. Let $X$ and $Y$ be members of $M$ such that $a \in X \cap Y$ and $b \in X-Y$. Then there exists a member $Z$ of $M$ such that $a \notin Z, b \in Z$ and $Z \subseteq X \cup Y$. 
If $M$ is indeed the polygon-matroid of a graph $G$, then $I$ is immediate from the properties of polygons. To show that II holds in this case we first observe that the polygon with edge-set $X$ contains an arc $L$ which has $b$ as an edge and has only its end-vertices $x$ and $y$ in common with the polygon of edge-set $Y$. But $x$ and $y$ are joined by an arc $L_{1}$ in the latter polygon such that $a$ is not an edge of $L_{1}$. Combining $L$ and $L_{1}$ we obtain a polygon whose edge-set $Z$ has the required properties. (See figure 1A.)

Conditions I and II are not sufficient for $M$ to be of the form $P(G)$. Before going any further it is convenient to lay down the following definition: a class $M$ of non-null subsets of a set $E$ is a matroid on $E$ if it satisfies conditions $I$ and $I I$. Thus these two conditions become the axioms of matroid theory.

Our first two theorems are as follows.

1.11 Let $L$ be a class of non-null subsets of a set $E$. Suppose L satisfies Axiom II. Then if a $\in X \in L$ there is a minimal member $Y$ of $L$ such that a $\epsilon Y \subseteq X$.

By a "minimal" member of $L$ we mean a member which does not contain another.

We shall denote the number of elements of any finite set $K$ by $|K|$.

To prove our theorem we define $Y$ as a member of $L$ satisfying $a \epsilon Y \subseteq X$ and having the least value of $|Y|$ consistent with this condition. We have to show that $Y$ is a minimal member of $L$.

Suppose not. Then there exists $Z \epsilon L$ such that $Z \subseteq Y-\{a\}$. Choose $b \in Z$. Then by Axiom II, with $a$ and $b$ interchanged, we find that there is a member $Z^{\prime}$ of $L$ such that $a \in Z^{\prime} \subseteq Y-\{b\} \subset X$. Since this contradicts the definition of $Y$ the theorem follows. 1.12 Let $L$ be a class of non-null subsets of a set $E$.

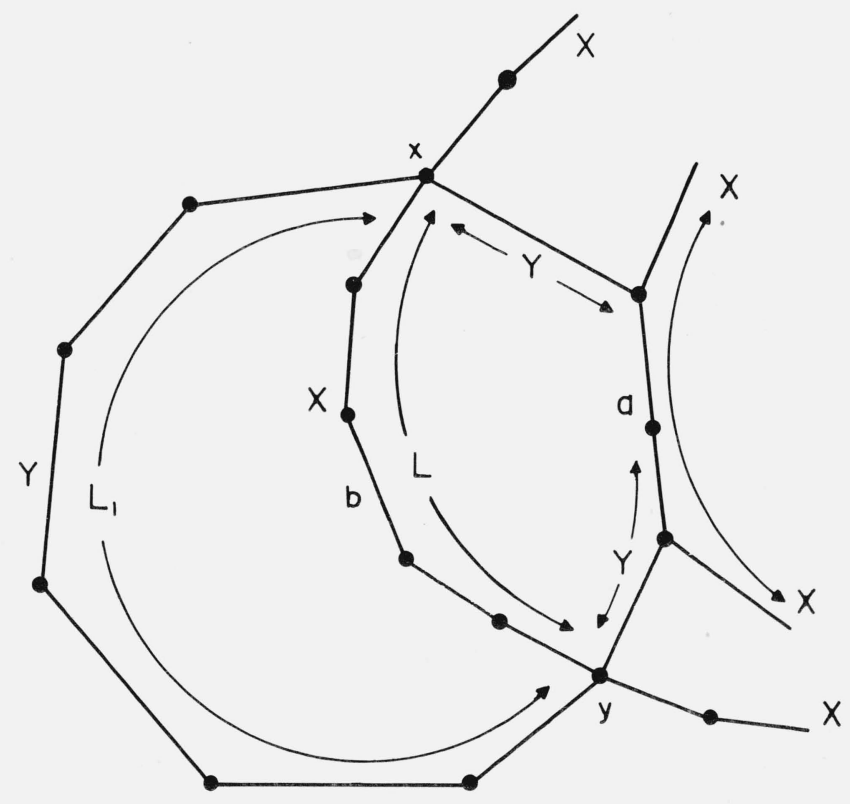

Figure 1A
Suppose L satisfies Axiom II. Then the minimal members of $L$ constitute a matroid on $E$.

Proof: Let $M$ be the class of minimal members of L. By definition it satisfies Axiom I.

Let $a$ and $b$ be members of $E$, and $X$ and $Y$ members of $M$, such that $a \in X \cap Y$ and $b \in X-Y$. By hypothesis there is a member $W$ of $L$ such that $a \notin W, b \in W$ and $W \subseteq X \cup Y$. By 1.1 there exists $Z \epsilon M$ such that $b \epsilon Z \subseteq W$. Hence $a \notin / Z, b \in Z$ and $Z \subseteq Z \cup Y$. Thus $M$ satisfies Axiom II.

Let $M$ be a matroid on a set $E$. We shall refer to the members of $E$ as the cells and to the members of $M$ as the atoms of the matroid.

We now describe some further examples of matroids.

\subsection{Chain-Groups}

Let $R$ be a commutative ring with a unit element and no divisors of zero. We define a chain on a finite set $E$ over $R$ as a mapping $f$ of $E$ into $R$. Thus each $a \epsilon E$ is associated with a number $f(a)$ in $R$, and this number may be called the coefficient of $a$ in the chain.

The domain $\|f\|$ of $f$ is the set of all members of $E$ with nonzero coefficients in $f$. If $\|f\|$ is null then $f$ is the zero chain on $E$ over $R$, denoted by the symbol 0 .

The sum $f+g$ of two chains $f$ and $g$ on $E$ over $R$ is another such chain defined as follows.

$$
(f+g)(a)=f(a)+g(a), \quad a \epsilon E .
$$

The product $\lambda f$ of a number $\lambda$ in $R$ and a chain $f$ on $E$ over $R$ is another chain on $E$ over $R$ defined as follows

$$
(\lambda f)(a)=\lambda(f(a)), \quad a \in E .
$$

We write $A(E, R)$ for the class of all chains on $E$ over $R$. A chain-group on $E$ over $R$ is defined as any subset $N$ of $A(E, R)$ which is closed under the operations of addition and multiplication by elements of $R$.

Let $N$ be such a chain-group. A chain $f$ of $N$ is called elementary if it is nonzero and there is no nonzero $g \in N$ such that $\|g\| \subset\|f\|$.

A primitive chain of $N$ is an elementary chain whose coefficients are restricted to the values 1,0 , and -1 in $R$.

We proceed to show that every chain-group has an associated matroid.

1.21 Let $N$ be a chain-group on $E$ over $R$. Then the class $M$ of the domains of the elementary chains of $N$ is a matroid on $E$.

Proof: Let $L$ be the class of domains of nonzero chains of $N$. Let $f$ and $g$ be two such chains and let $a$ and $b$ be elements of $E$ ("cells" of $N$ ) such that $a \epsilon|| f|| \cap\|g\|$ and $b \epsilon|| f||-\|g\|$.

We multiply $f$ by $g(a)$ and $g$ by $-f(a)$ and add the resulting chains. We thus obtain a chain $h$ of $N$ such that $a \notin\|h\|, b \epsilon\|h\|$ and $\|h\| \subset\|f\| \cup\|g\|$. We deduce that $L$ satisfies Axiom II.

But $M$ is the class of minimal members of $L$. Hence $M$ is a matroid on $E$ by 1.2 . 
We call $M$ the matroid of $N$ and denote it by $M(N)$.

A chain group over the ring of residues $\bmod 2$ is called binary, and one over the ring of integers is called integral. A regular, or completely unimodular chain-group is an integral chain-group in which each elementary chain is a multiple of a primitive chain.

A matroid is called binary or regular if it is the matroid of a binary or regular chain-group respectively. These two kinds of matroid are of special importance in the theory.

We conclude this chapter by showing how to associate the polygon-matroid of a graph $G$ with a chain-group over $R$.

We first assign an orientation to each edge of $G$ by distinguishing one end as positive and the other as negative. For each $a \in E(G)$ and each $x \in V(G)$ we define an integer $\eta(a, x)$ as follows. If $a$ and $x$ are not incident, or $a$ is a loop, then $\eta(a, x)=0$. Otherwise $\eta(a, x)=1$ or -1 according as $x$ is the positive or negative end of $a$.

Chains on $V(G)$ and $E(G)$ are called 0-chains and 1chains of $G$ respectively.

Let $f$ be a 1-chain of $G$ over $R$. We define a 0 -chain $\partial f$ of $G$ over $R$, called the boundary of $f$, by the following rule

$$
(\partial f)(x)=\sum_{a \in E(G)} \eta(a, x) f(a), \quad x \in V(G) .
$$

We call $f$ a $l$-cycle of $G$ if $\partial f=0$. It is clear that the l-cycles of $G$ over $R$ constitute a chain-group $N$ on $E(G)$ over $R$.

Given a polygon of $G$ we can easily construct a 1 cycle of $G$, with coefficients $1,-1$ and 0 whose domain is the edge-set of the polygon. On the other hand let $f$ be any nonzero 1-cycle over $R$, and let $G_{f}$ be the subgraph of $G$ made up of the edges of $\|f\|$ and their incident vertices. Then the valency of each vertex of $G_{f}$ exceeds 1 , and therefore $G_{f}$ contains a polygon. From these observations we have

1.22 Let $N$ be the chain-group of 1 -cycles over $R$ for an oriented graph $G$. Then $M(N)=P(G)$.

In particular $R$ may be the ring of residues $\bmod 2$. Hence

1.23 The polygon-matroid of a graph $G$ is binary.

We have seen that the edge-set of any polygon is the domain of a primitive chain of $N$. It readily follows that

1.24 The polygon-matroid of a graph $G$ is regular.

\section{Duality}

\subsection{Principal Forests}

A spanning tree of a graph $G$ is a subgraph of $G$ which is a tree and which includes all the vertices of $G$. A principal forest of $G$ is a subgraph of $G$ whose intersection with each component of $G$ is a spanning tree of that component. For our purposes the main theorem about principal forests is the following. 2.11 Let $U$ be a subset of $E(G)$. Then $U$ is complementary to the edge-set of a principal forest of $G$ if and only if it has the following properties:

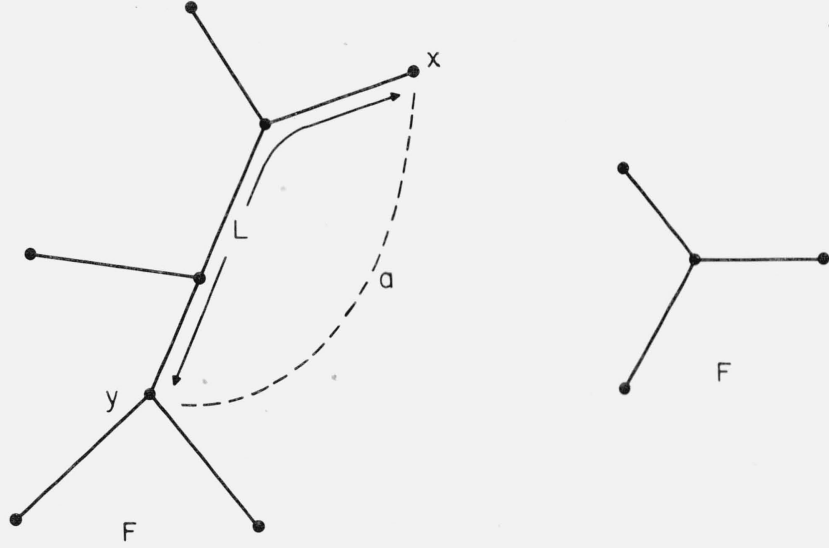

Figure 2A

(i) $U$ meets the edge-set of each polygon of $G$.

(ii) No proper subset of $U$ meets the edge-set of every polygon of $G$.

Proof: Suppose first that $U$ has properties (i) and (ii). Let $F$ be the spanning subgraph of $G$ such that $E(F)=E(G)-U$. Then $F$ is a forest, by (i). Suppose its intersection with some component $C$ of $G$ is not connected. Then some edge $a \epsilon E(C) \cap U$ has its ends in different components of $F$. But then there can be no polygon of $G$ whose edge-set does not meet $U-\{a\}$, contrary to (ii).

Conversely suppose $U$ is complementary to the edge-set of a principal forest $F$. Then $F$ has no polygon. But if $a$ is an edge of $U$ with ends $x$ and $y$, then either $x=y$ or $x$ and $y$ are joined in $F$ by an $\operatorname{arc} L$. (See figure 2A). In the latter case $L$ can be combined with $a$ to form a polygon whose edge-set does not meet $U-\{a\}$. Hence $U$ satisfies (i) and (ii).

\subsection{Dendroids}

We can now make an extension to matroid theory as follows. Let $M$ be a matroid on a set $E$. Then we define a dendroid $D$ of $M$ as a subset of $E$ which meets every atom of $M$ and is minimal with respect to this property. Thus

2.21 The dendroids of $P(G)$ are the complements in $E(G)$ of the edge-sets of the principal forests of $G$.

Let $D$ be a dendroid of $M$ and let $a$ be a cell of $D$. By the definition of a dendroid there is an atom $X$ of $M$ such that $X \cap D=\{a\}$. Moreover $X$ is unique. For suppose $Y$ is another atom of $M$ such that $Y \cap D=\{a\}$. Then an application of Axiom II shows that there is an atom $Z$ of $M$ not meeting $D$. We denote $X$ by $J(D, a)$.

We now give some general theorems about dendroids of $M$.

2.22 Let $D$ be a dendroid of $M$. Let $a$ and $b$ be cells of $M$ such that $a \epsilon D$ and $b \notin D$. Write $D^{\prime}=(D-\{a\}) \cup\{b\}$. Then $D^{\prime}$ is a dendroid of $M$ if and only if $b \in J(D, a)$.

Proof: Suppose $D^{\prime}$ is a dendroid of $M$. Since it meets $J(D, a)$ we must have $b \epsilon J(D, a)$. 
Conversely suppose $b \in J(D, a)$. Then $D^{\prime}$ meets every atom of $M$, since $D-\{a\}$ meets every such atom other than $J(D, a)$.

We note that $D^{\prime}-\{b\}$ does not meet $J(D, a)$. Consider any cell $c \epsilon D^{\prime}-\{b\}$. Then $D^{\prime} \cap J(D, c)$ is either $\{c\}$ or $\{b, c\}$. In the second alternative an application of Axiom II to $J(D, a)$ and $J(D, c)$ shows that $M$ has an atom $Z$ meeting $D^{\prime}$ solely in $\{c\}$. We conclude that no proper subset of $D^{\prime}$ meets every atom of $Z$. The theorem follows.

2.23 All dendroids of $M$ have the same number of cells.

Proof: Let $D_{1}$ and $D_{2}$ be distinct dendroids of $M$. Let $D_{3}$ be a dendroid of $M$ such that $\left|D_{3}\right|=\left|D_{1}\right|$ and $\left|D_{2} \cap D_{3}\right|$ has the greatest value consistent with this condition.

If possible choose $a \epsilon D_{3}-D_{2}$. Then $J\left(D_{3}, a\right)$ meets $D_{2}$ in a cell $b$ which is not in $D_{3}$. Since $\left(D_{3}-\{a\}\right) \cup\{b\}$ is a dendroid of $M$, by 2.21 , the definition of $D_{3}$ is contradicted.

We deduce that $D_{3} \subseteq D_{2}$, and therefore $D_{3}=D_{2}$. Hence $\left|D_{1}\right|=\left|D_{2}\right|$, and the theorem follows.

The number of cells in a dendroid of $M$ is the rank $r(M)$ of $M$. In the trivial case in which $M$ has no atoms there is just one dendroid, the null subset of $E$, and $r(M)=0$. In every other case $r(M)>0$.

2.24 Let $X$ be an atom of $M$. Then there is a dendroid $D$ of $M$ and a cell $a$ of $D$ such that $X=J(D, a)$.

Proof: Let $D$ be a dendroid of $M$ with the least possible value of $|D \cap X|$. Suppose $D \cap X$ has two distinct cells $a$ and $b$. Then $J(D, b)$ has a cell $c$ not in $X$, by Axiom I. But $(D-\{b\}) \cup\{c\}$ is a dendroid of $M$, by 2.3 , which is contrary to the definition of $D$. We conclude that $D \cap X$ consists of a single cell $a$, so that $X=J(D, a)$.

Corollary. If $x \in X$ there is a dendroid $D^{\prime}$ of $M$ such that $X=J\left(D^{\prime}, x\right) . \quad(2.22$ and 2.24$)$.

$2.25 A$ matroid $M$ on a given set $E$ is uniquely determined by its dendroids.

Proof: If $D$ is any dendroid of $M$ and $a \epsilon D$, then $J(D, a)$ is uniquely determined by the other dendroids, by 2.22 . Hence $M$ is completely determined by its dendroids, by 2.24 .

\subsection{Dendroids of a Chain-Group}

The concept of a dendroid can be applied to a chaingroup $N$ on $E$ over a commutative $\operatorname{ring} R$ of the type described in chapter I. We define a dendroid of $N$ as a minimal subset $D$ of $E$ meeting the domain of every nonzero chain of $N$. Since every such domain contains that of an elementary chain the dendroids of $N$ are identical with those of the corresponding matroid $M(N)$.

Let $D$ be a dendroid of $N$. For each $a \epsilon D$ we can find a chain $f_{a}$ of $N$ such that $\left\|f_{a}\right\|$ is the atom $J(D, a)$ of $M(N)$. We refer to the set of chains $f_{a}$, one for each $a \epsilon D$, as a dendroid-basis of $N$. Its weight is the product

$$
\prod_{a \in D} f_{a}(a),
$$

which is necessarily nonzero.
2.31 Let $B=\left\{\mathrm{f}_{\mathrm{a}} \mid \mathrm{a} \in D\right\}$ be $a$ dendroid-basis of $N$ of weight $w$. Let $K$ be any non-zero chain of $N$. Then $w K$ can be expressed as a linear combination of members of $B$, with multipliers in $R$.

Proof: By adding suitable multiples of members of $B$ to $w K$ we can reduce the coefficient of each $a \epsilon D$ to zero. The resulting chain is zero, since its domain does not meet $D$.

COROLlary: If $\|K\|$ meets $D$ in a single cell a, then $\|K\|=\left\|f_{a}\right\|$, and therefore $K$ is an elementary chain of $N$.

\subsection{Dual Chain-Groups}

We note that the rank of $M(N)$ is also the rank of $N$, in the sense of the theory of linear dependence, by 2.31. Much simplification results if we can arrange that $f_{a}(a)=1$ for each $a \epsilon D$. This can be done when $R$ is a field and also when $N$ is regular. In the latter case $f_{a}$ is taken to be a primitive chain of $N$. In these cases the weight of the dendroid-basis takes the value 1. The dendroid-basis then becomes a true basis of $N$, by 2.31 .

Returning to the general case we define two chains $f$ and $g$ on $E$ over $R$ as orthogonal if

$$
\sum_{a \in E} f(a) g(a)=0
$$

Let $N^{*}$ be the class of all chains $h$ on $E$ over $R$ such that $h$ is orthogonal to every member of $N$. It is easily verified that $N^{*}$ is a chain-group on $E$ over $R$. We call it the dual chain-group of $N$. It is clear from the definition that

$$
N \subseteq N^{* *}
$$

2.42 Let $D$ be a dendroid of $N$ and let $B=\left\{f_{a} / a \epsilon D\right\}$ be a corresponding dendroid-basis of $N$.

For each $b \in E-D$ let a chain $g_{b}$ on $E$ be defined as follows: $g_{b}(b)$ is the weight $w(B)$ of $B$, and $g_{b}(x)=0$ for each $x \in(E-D)-\{b\}$. On the other hand if $a \in D$, then

$$
g_{b}(a)=-f_{a}(b) \underset{c \in D-\{a\}}{\prod} f_{c}(c) .
$$

Then $E-D$ is a dendroid of $N^{*}$, and the set $B^{*}$ $=\left\{g_{b} \mid b \in E-D\right\}$ is a corresponding dendroid-basis of $N^{*}$.

Proof: For each $g_{b} \in B^{*}$ it is readily verified that $g_{b}$ is orthogonal to each $f_{\mathrm{a}} \in B$. Hence $g_{b}$ is orthogonal to each chain of $N$, by 2.31 and the fact that $R$ has no divisors of zero. Hence $g_{b} \in N^{*}$.

A chain on $E$ over $R$ whose domain is a non-null subset of $D$ cannot be orthogonal to every member of $B$. Hence $E-D$ meets the domain of every nonzero chain of $N^{*}$. Considering the chains $g_{b}$ we see that no proper subset of $E-D$ has this property. Hence $E-D$ is a dendroid of $N^{*}$. By 2.31, Corollary, $B^{*}$ is a corresponding dendroid-basis.

2.43 The dendroids of $N^{*}$ are the complements in $E$ of the dendroids of $N$. 
Proof: Any dendroid of $N$ is also a dendroid of $N^{* *}$, by two applications of 2.42. Hence $N$ and $N^{* *}$ have equal ranks.

Let $E-U$ be any dendroid of $N^{*}$. Then $U$ is a dendroid of $N^{* *}$, by 2.42. It contains a dendroid $U^{\prime}$ of $N$, by 2.41 , and $U^{\prime}=U$ by the result of the preceding paragraph. Thus $U$ is a dendroid of $N$. Combining this result with 2.42 we establish the theorem.

\subsection{4}

$$
M\left(N^{* *}\right)=M(N),
$$

by 2.25 and 2.43 .

2.45 Any dendroid-basis of $N$ is a dendroid-basis of $N^{* *}$, by $2.41,2.44$ and 2.31 , Corollary.

2.46 If $N$ has a dendroid-basis of weight 1 , then $N^{* *}=N$, by 2.31 and 2.45 .

2.47 If $N$ is regular, then $N^{*}$ is regular.

Proof: We may suppose the chains $f_{a}$ of 2.42 to be primitive. Then the chains $g_{b}$ have coefficients restricted to the values 0,1 and -1 . Since they belong to a dendroid-basis of $N^{*}$ they are elementary and therefore primitive chains of $N^{*}$. Using 2.24 we deduce that $N^{*}$ is regular.

\subsection{Bond-Matroid of a Graph}

Suppose now that $N$ is the group of 1-cycles on a graph $G$ over $R$. Then the dendroids of $N^{*}$ are the edge-sets of the principal forests of $G$, by $1.22,2.21$, and 2.43. We proceed to interpret $N^{*}$ in terms of the structure of $G$.

Let $g$ be any 0-chain on $G$ over $R$. Then the coboundary $\delta g$ of $g$ is a 1-chain on $G$ over $R$ defined as follows

$$
(\delta g)(a)=\sum_{x \in V(G)} \eta(a, x) g(x), \quad a \in E(G) .
$$

It is clear that the coboundaries of the 0-chains on $G$ over $R$ are the elements of a chain-group $N_{1}$ on $E(G)$ over $R$.

2.51 Every coboundary of $G$ over $R$ is orthogonal to every 1 -cycle of $G$ over $R$.

Proof: Let $k$ be a l-cycle and $f$ the coboundary of a 0 -chain $g$. Then

$$
\sum_{a \in E(G)} k(a) f(a)=\sum_{a \in E(G)} \sum_{x \in V(G)} \eta(a, x) k(a) g(x)=\sum_{x \in V(G)} g(x) h(x),
$$

where $h=\partial k$. Hence

$$
\sum_{a \in E(G)} k(a) f(a)=0 .
$$

As a corollary we note that $N_{1} \subseteq N^{*}$.

Now let $F$ be a dendroid of $N^{*}$, the edge-set of a principal forest of $G$.

Consider any $a \epsilon F$, belonging to a component $C_{a}$ of $G$. The intersection of the principal forest with $C_{a}$ is a tree $T$ having $a$ as an edge. When $a$ is deleted $T$ is decomposed into two components $T_{1}$ and $T_{2}$, which we may suppose to contain the positive and negative ends of $a$ respectively. (See figure 2B.)

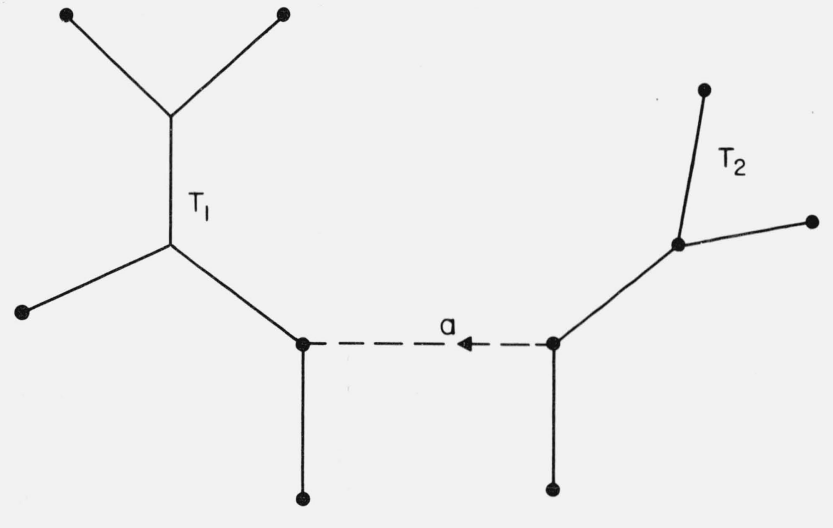

Figure 2B

Let $g_{a}$ be the 0 -chain over $R$ in which the vertices of $T_{1}$ have coefficient 1 and all other vertices coefficient 0 . Consider the coboundary $f_{a}=\delta g_{a}$. Clearly $f_{a}(a)=1$ and the other coefficients in $f_{a}$ are restricted to the values $1,-1$ and 0 . Moreover $F \cap\left\|f_{a}\right\|=\{a\}$. The last result implies, since $N_{1} \subseteq N^{*}$, that $f_{a}$ is an elementary chain of $N^{*}$, by 2.31 , Corollary.

The coboundaries $f_{a}, a \epsilon F$, thus constitute a dendroid-basis of $N^{*}$. Using 2.31 we obtain

\section{$2.52 N^{*}$ is the chain-group of coboundaries of $G$ over $R$}

We refer to $M\left(N^{*}\right)$ as the bond-matroid $B(G)$ of $G$. We note that in the above proof $\left\|f_{a}\right\|$ is not dependent on the particular ring $R$ used. Hence, by 2.24 , Corollary, $B(G)$ is independent of $R$.

Since $R$ may be the ring of residues $\bmod 2$ we have $2.53 B(G)$ is binary.

If $R$ is the ring of integers we observe that the chains $f_{a}$ are all primitive. Hence, by 2.24 , Corollary, we have $2.54 B(G)$ is regular.

We refer to the atoms of $B(G)$ as the bonds of $G$. As an exercise in graph theory we can show that a nonnull subset $K$ of $E(G)$ is a bond of $G$ if and only if it satisfies the following condition: the graph $H$ obtained from $G$ by deleting the edges of $K$ has two components $C_{1}$ and $C_{2}$ such that each edge of $K$ has one end in each.

We have adopted a nomenclature which treats $B(G)$ as more fundamental then $P(G)$. Thus principal forests of $G$ correspond directly to "dendroids" of $B(G)$. We also describe a matroid as graphic if it can be represented as the bond-matroid of a graph, and as cographic if it can be represented as a polygon-matroid.

There is a theory of dual graphs on the 2-sphere, but we shall comment only briefly about it. Two graphs $G$ and $H$ may be defined as dual if there is a $1-1$ mapping of $E(G)$ onto $E(H)$ which transforms 1-cycles into coboundaries, and coboundaries into 1-cycles. It can be shown that a graph is planar, that is realizable in the 2 -sphere, if and only if it has a dual graph. Using this theory we may assert that a graph $G$ is planar if and only if its polygon-matroid is graphic. Let us 
therefore define a planar matroid as one which is both graphic and cographic. Such a matroid corresponds to a pair of dual planar graphs.

\subsection{Dual Matroids}

There is a theory of duality for general matroids which is closely analogous to that given for chaingroups in section 2.4 .

Two subsets $S$ and $T$ of a finite set $E$ are called orthogonal if $|S \cap T| \neq 1$. Given a matroid $M$ on $E$ we denote the class of all non-null subsets of $E$ which are orthogonal to every atom of $M$, by $L(M)$.

2.61 If $(X, Y) \in L(M), a \in X \cap Y$ and $b \in X-Y$, then there exists $Z \epsilon L(M)$ such that $b \in Z \subseteq(X \cup Y)-\{a\}$.

Proof: Assume the theorem false for some $X, Y, a, b$. We construct a sequence $\left(a_{0}, a_{1}, \ldots, a_{k}\right)$ of cells of $X \cup Y$, and a sequence $\left(T_{1}, T_{2}, \ldots, T_{k}\right)$ of atoms of $M$ as follows. First we put $a_{0}=a$. If we have determined the cells $a_{i}$ as far as $a_{r}$, and $b$ is not among them we take $T_{r+1}$ to be any atom of $M$ such that

$$
T_{r+1} \cap\left\{(X \cup Y)-\left\{a_{0}, \ldots, a_{r}\right\}\right\}
$$

has just one cell, $c$ say. This is possible since $(X \cup Y)$ $-\left\{a_{0}, \ldots, a_{r}\right\}$ is not in $L(M)$, by assumption. We then write $c=a_{r+1}$. The construction terminates with $a_{k}=b$.

There exists $U \epsilon M$ such that $b \epsilon U \cap(X \cup Y) \subseteq\left\{a_{0}\right.$, . . ., $\left.a_{k}\right\}$. For example we may take $U=T_{k}$. For each such $U$ let $p(U)$ be the greatest integer $j$ less than $k$ such that $a_{j} \epsilon U$. Such an integer exists since $|U \cap X| \neq 1$.

Suppose $p(U)>0$. We apply Axiom II to $U$ and $T_{p(U)}$, thus establishing the existence of an atom $U^{\prime}$ of $M$ such that

$$
b \epsilon U^{\prime} \subseteq\left(U \cup T_{p(U)}-\left\{a_{p(U)}\right\}\right)
$$

But then $b \epsilon U^{\prime} \cap(X \cup Y) \subseteq\left\{a_{0}, \ldots, a_{k}\right\}$, and $p\left(U^{\prime}\right)$ $<p(U)$.

We can therefore choose $U$ so that $p(U)=0$. But then $Y \cap U=\left\{a_{0}\right\}=\{a\}$, contrary to hypothesis. The theorem follows.

We denote the class of minimal members of $L(M)$ by $M^{*}$. Now 2.61 asserts that $L(M)$ satisfies Axiom II. Hence,

$2.62 M^{*}$ is a matroid on $E$.

by 1.12. We call $M$ the dual matroid of $M$. From the definition of orthogonality we see that

$$
M \subseteq M^{* *} .
$$

If $D$ is a dendroid of $M$ and $b \in E-D$ we define $K(D, b)$ as the subset of $E$ consisting of $b$ and each $a \epsilon D$ such that $b \in J(D, a)$.

$2.63 K(D, b) \epsilon M^{*}$.

Proof: Assume $K(D, b)$ is not in $L(M)$. Then there exists an atom $X$ of $M$ such that $|X \cap K(D, b)|=1$. Choose such an $X$ so that $|D \cap X|$ has the least possible value, and denote the common cell of $X$ and $K(D, b)$ by $c$.
Suppose $D \cap X$ has a cell $e$ not in $K(D, b)$. Applying Axiom II to $X$ and $J(D, e)$ we find that $M$ has an atom $Y$ such that $c \epsilon Y \subseteq(X \cup J(D, e))-\{e\}$. But then $Y \cap K(D, b)$ $=\{c\}$, since $\bar{b} \notin J(D, e)$. Moreover $|D \cap Y|<|D \cap X|$. This is contrary to the definition of $X$.

We deduce that $D \cap X \subset X \cap K(D, b)=\{c\}$. Hence, since $D$ is a dendroid of $\bar{M}, D \cap X=\{c\}$ and therefore $X=J(D, c)$. But $c \in K(D, b)$ and therefore $b \in J(D, c)$. Hence $X \cap K(D, b)$ includes two distinct cells $b$ and $c$, which is contrary to the definition of $X$. Hence $K(D, b)$ is in fact in $L(M)$.

There is an atom $U$ of $M^{*}$ such that $b \epsilon U \subseteq K(D, b)$, by 1.11. But if $x \in K(D, b)-U$, then $U$ is not orthogonal to $J(D, x)$. Hence $K(D, b)=U \epsilon M^{*}$.

Corollary: $E-D$ is a dendroid of $M^{*}$, and $K(D, b)$ is the atom $J(E-D, b)$ of $M^{*}$.

2.64 The dendroids of $M^{*}$ are the complements in $E$ of the dendroids of $M$.

Proof: The proof is analogous to that of 2.43 . We note that any dendroid of $M$ is a dendroid of $M^{* *}$, by 2.63 , Corollary. Hence $r\left(M^{* *}\right)=r(M)$.

Let $E-U$ be any dendroid of $M^{*}$. Then $U$ is a dendroid of $M^{* *}$. It contains a dendroid $U^{\prime}$ of $M$, by (1). But then $U^{\prime}=U$ by the equality of rank.

Combining this result with the Corollary to 2.63 we establish the theorem.

2.65

$$
M^{* *}=M,
$$

by 2.64 and 2.25 .

2.66 Let $N$ be a chain-group on $E$ over a commutative ring $R$ with a unit element and no divisors of zero. Then

$$
(M(N))^{*}=M\left(N^{*}\right),
$$

by $2.43,2.64$ and 2.25 . In particular we have 2.67 For any finite graph $G$,

$$
(P(G))^{*}=B(G),
$$

by 2.52 .

\section{Structure of Matroids}

\subsection{Subgraphs and Contractions}

Let $G$ be a graph and let $S$ be a subset of $E(G)$. There are two specially important subgraphs of $G$ associated with $S$. One is the spanning subgraph $G: S$, for which $V(G: S)=V(G)$ and $E(G: S)=S$. The other is the reduction of $G$ to $S$, defined by the edges of $G$ and their incident vertices. We denote it by $G \cdot S$.

We note that $G \cdot S$ has no isolated vertices. For this reason we shall usually prefer the reductions of $G$ to the other subgraphs. For the addition or removal of an isolated vertex in a graph $G$ does not affect the 1-cycles or coboundaries.

The contraction $G$ ctr $S$ of $G$ to $S$ is a graph whose vertices are the components of $G:(E(G)-S)$ and whose edges are the members of $S$. The ends of an edge in $G \operatorname{ctr} S$ are the two components, possibly coincident, of $G:(E(G)-S)$ which include the ends of the edge in $G$. 
If the identities of the vertices of $G$ are not important we may say, less precisely, that $G \operatorname{ctr} S$ is formed from $G$ by contracting each component of $G:(E(G)-S)$ to a single vertex. Alternatively we may say that $G$ ctr $S$ is obtained from $G$ by a sequence of "elementary contractions" in each of which one edge of $G$ is contracted to a single vertex.

By deleting the isolated vertices, if any, of $G$ ctr $S$ we obtain from it the reduced contraction $G \times S$ $=(G \operatorname{ctr} S) \cdot S$ of $G$ to $S$.

We go on to derive some corresponding operations on chain-groups and matroids.

\subsection{Minors of Chain-Groups}

Let $N$ be a chain-group on a set $E$ over a commutative ring $R$ having a unit element and no divisors of zero.

Suppose $S \subseteq E$. If $f$ is any chain of $N$ we define its restriction to $S$ as the chain $g$ on $S$ such that $g(a)$ $=f(a)$ for each $a \epsilon S$.

The restrictions to $S$ of the chains of $N$ constitute a chain-group on $S$ over $R$. We call this the reduction of $N$ to $S$ and denote it by $N \cdot S$. Another chain-group on $S$ over $R$ is given by the restrictions to $S$ of those chains $f$ of $N$ for which $\|f\| \subseteq S$. This is the contraction of $N$ to $S$, denoted by $N \times S$.

$3.21 N \times S \subseteq N \cdot S$.

The proofs of the two following theorems are simple exercises in graph theory.

3.22 Let $N$ be the group of coboundaries over $R$ of a graph $G$. Then $N \cdot S$ is the group of coboundaries over $R$ of $G \cdot S$.

3.23 Let $N$ be as in 3.22. Then $N \times S$ is the group of coboundaries over $R$ of $G \times S$.

Returning to the general case we suppose $T \subseteq S \subseteq E$. We can now establish the following identities

$$
(N \times S) \times T=N \times T,
$$

$$
\begin{gathered}
(N \cdot S) \cdot T=N \cdot T, \\
(N \cdot S) \times T=(N \times(E-(S-T))) \cdot T, \\
(N \times S) \cdot T=(N \cdot(E-(S-T))) \times T .
\end{gathered}
$$

The first two of these follow immediately from the definitions. To prove 3.243 we observe that each side of the formula represents the restrictions to $T$ of those chains of $N$ which have only zero coefficients in $S-T$. We can obtain 3.244 by writing $E-(S-T)$ for $S$ in 3.243 .

We refer to a chain-group of the form $(N \cdot S) \times T$ as a minor of $N$. The minors of $N$ include $N$ itself and all its reductions and contractions. For $N=(N \cdot E) \times E$, $N \cdot S=(N \cdot S) \times S$, and $N \times S=(N \cdot E) \times S$. From the identities 3.241 we deduce

3.25 Every minor of a minor of $N$ is a minor of $N$.

3.26 If $N$ is regular then every minor of $N$ is regular.

Proof: Suppose $S \subseteq E$. Let $f$ be an elementary chain of $N \times S$. Then $f$ is the restriction to $S$ of an elementary chain $f_{1}$ of $N$ having the same domain as $f$. But $f_{1}$ is an integral multiple of a primitive chain of $N$, since $N$ is regular. This implies that $f$ is an integral multiple of a primitive chain of $N \times S$. We deduce that $N \times S$ is regular.

Now let $h$ be an elementary chain of $N \cdot S$. There is a chain $h_{1}$ of $N$ having $h$ as its restriction to $S$. Choose $h_{1}$ so that $\left\|h_{1}\right\|$ has the least possible number of cells. There is an elementary chain $h_{2}$ of $N$ such that $\left\|h_{2}\right\|$ $\subseteq\left\|h_{1}\right\|$. By the choice of $h_{1}$ we may suppose $\left\|h_{2}\right\| \cap S$ is non-null. Since $h_{2}$ is a multiple of a primitive chain of $N$ we deduce that there is a nonzero chain $k$ of $N \cdot S$, with coefficients restricted to the values $1,-1$ and 0 , such that $\|k\| \subseteq\|h\|$. But then $\|k\|=\|h\|$ since $h$ is elementary, and therefore $k$ is a primitive chain of $N \cdot S$. Since $h$ is elementary it must be a multiple of $k$. We deduce that $N \cdot S$ is regular.

The theorem follows from these two results.

Minors of dual chain-groups are related by the following identity.

$$
(N \cdot S)^{*}=N^{*} \times S .
$$

Proof: Let $f$ be a chain on $S$. It belongs to $(N \cdot S)^{*}$ if and only if it is orthogonal to every chain of $N \cdot S$, that is if and only if the chain $g$ on $E$ which satisfies $\|g\|=\|f\|$ and has $f$ as its restriction to $S$ is orthogonal to every member of $N$. But this condition holds if and only if $g \in N^{*}$, that is $f \in N^{*} \times S$.

In the cases of chief interest to us we can supplement this result as follows.

3.28 Suppose that $R$ is a field or that $N$ is regular. Then

$$
(N \times S)^{*}=N^{*} \cdot S,
$$

for each $S \subset E$.

Proof: $\overline{\mathrm{W}}$ riting $N^{*}$ for $N$ in 3.27 , and then taking dual chain-groups we obtain

$$
\left(N^{* *} \times S\right)^{*}=\left(N^{*} \cdot S\right)^{* *} .
$$

But under the conditions of the theorem $N^{* *}=N$, by 2.46. Similarly $\left(N^{*} \cdot S\right)^{* *}=N^{*} \cdot S$, by 2.47 and 3.26 . The theorem follows

3.29 Under the conditions of 3.28 the duals of the minors of $N$ are the minors of $N^{*}$ (by 3.27 and 3.28).

\subsection{Minors of Matroids}

This section is closely analogous to 3.2 .

Let $M$ be a matroid on a set $E$, and suppose $S \subseteq E$.

Let $L$ be the class of non-null intersections with $S$ of atoms of $M$, and let $M \cdot S$ be the class of minimal members of $L$. Since $M$ satisfies Axiom II it is clear that $L$ does so too. Hence $M \cdot S$ is a matroid on $S$, by 1.12 . We call it the reduction of $M$ to $S$.

Let $M \times S$ be the class of all atoms $X$ of $M$ such that $X \subseteq S$. Then $M \times S$ satisfies Axioms I and II. It is thus a matroid on $S$. We call it the contraction of $M$ to $S$.

By a comparison of definitions we have the following theorem 
3.31 Let $N$ be a chain-group on $E$ over a ring $R$ with a unit element and no divisors of zero. Then

$$
\begin{gathered}
M(N \times S)=M(N) \times S, \\
M(N \cdot S)=M(N) \cdot S .
\end{gathered}
$$

Now take $N$ to be the group of coboundaries over $R$ of a graph $G$. Then by $3.22,3.23$ and 3.31 we have

$$
\begin{gathered}
B(G \cdot S)=B(G) \cdot S, \\
B(G \times S)=B(G) \times S .
\end{gathered}
$$

Here $B(G)$ is the bond-matroid of $G$ defined in section 2.5 .

Returning to the general case we suppose $T \subseteq S \subseteq E$.

We establish the following identities

$$
\begin{array}{lc}
3.331 & (M \times S) \times T=M \times T, \\
3.332 & (M \cdot S) \cdot T=M \cdot T, \\
3.333 & (M \cdot S) \times T=(M \times(E-(S-T))) \cdot T, \\
3.334 & (M \times S) \cdot T=(M \cdot(E-(S-T))) \times T .
\end{array}
$$

The first of these follows at once from the definitions.

To prove 3.332 we observe that if $X \epsilon(M \cdot S) \cdot T$ then $X$ is a non-null intersection with $T$ of an atom of $M \cdot S$, and therefore a non-null intersection with $T$ of an atom of $M$. Hence there exists $Y \epsilon M \cdot T$ such that $Y \subseteq X$. Conversely suppose $Y \epsilon M \cdot T$. Then $Y$ is a non-null intersection with $T$ of an atom $Z$ of $M$. But $Z$ contains an atom $Z_{1}$ of $M \cdot S$ meeting $T$, by 1.11. Similarly $Z_{1}$ contains an atom $X$ of $(M \cdot S) \cdot T$, and we have $X \subseteq Y$. Applying Axiom I to these results we have 3.332 .

We prove 3.333 in a similar way. Suppose $X \epsilon$ $(M \cdot S) \times T$. Then $X$ is an atom of $M \cdot S$ contained in $T$. Accordingly $M$ has an atom $X_{1}$ such that $X_{1} \cap T=X$ and $X_{1} \cap(S-T)$ is null. But then $X_{1}$ is an atom of $M \times(E-(S-T))$ and hence there is an atom $Y$ of $(M \times(E-(S-T))) \cdot T$ such that $Y \subseteq X$. Conversely suppose $Y \epsilon(M \times(E-(S-T))) \cdot T$. Then $Y$ is the intersection with $T$ of an atom $Y_{1}$ of $M \times(E-(S-T))$, and $Y_{1}$ is an atom of $M$ such that $Y_{1} \cap(S-T)$ is null. Hence there is an atom $X$ of $M \cdot S$ which is contained in $Y_{1}$. We have in fact $X \subseteq Y \subseteq T$. Hence $(M \cdot S) \times T$ has an atom $X$ such that $X \subseteq Y$. Applying Axiom I to these results we establish 3.333 .

We obtain 3.334 by writing $E-(S-T)$ for $S$ in 3.333 .

We refer to a matroid of the form $(M \cdot S) \times T$ as a minor of $M$. The minors of $M$ evidently include $M$ itself and all its reductions and contractions. From the four identities just proved we deduce

3.34 Every minor of a minor of $M$ is a minor of $M$.

Minors of dual matroids are related by the following identities
3.351
$(M \cdot S)^{*}=M^{*} \times S$,
3.352
$(M \times S)^{*}=M^{*} \cdot S$.

To prove 3.351 suppose $X \epsilon(M \cdot S)^{*}$. Then $X \subseteq S$ and $X$ is orthogonal to every atom of $M \cdot S$. Now the class of intersections with $S$ of atoms of $M$ satisfies Axiom II and its minimal members constitute $M \cdot S$. If $X$ meets one of these intersections in a single cell, then it meets some atom of $M \cdot S$ in a single cell, by 1.11. Hence $X$ is orthogonal to every atom of $M$. Accordingly there exists $Y \epsilon M^{*}$ such that $Y \subseteq X$. But then $Y \epsilon M^{*} \times S$.

Conversely suppose $Y \epsilon M^{*} \times S$. Then $Y \subseteq S$ and $Y \epsilon M^{*}$. Hence $Y$ is orthogonal to every member of $M$ and therefore to every member of $M \cdot S$. Accordingly there exists $X \epsilon(M \cdot S)$ * such that $X \subseteq Y$.

Applying Axiom I to these results we obtain 3.351.

Writing $M^{*}$ for $M$ in 3.351 and taking dual matroids we obtain

$$
\left(M^{*} \cdot S\right)^{* *}=\left(M^{* *} \times S\right)^{*}
$$

By 2.65 this reduces to 3.352 .

3.36 The minors of $M^{*}$ are the duals of the minors of $M$.

This follows from 3.351 and 3.352 , with the help of 3.334 .

The foregoing results can be applied to the bondmatroid and polygon-matroid of a graph $G$, these being duals by 2.67. On taking the dual forms of 3.321 and 3.322 we obtain the following identities.

$$
\begin{array}{ll}
3.371 & P(G \cdot S)=P(G) \times S, \\
3.372 & P(G \times S)=P(G) \cdot S .
\end{array}
$$

\subsection{Connection in Matroids}

Consider a matroid $M$ on a set $E$. We define a separator of $M$ as a subset $S$ of $E$ such that each atom of $M$ is contained either in $S$ or in $E-S$. Evidently any union or intersection of separators of $M$ is a separator of $M$, and the complement of a separator of $M$ is also a separator.

We refer to the minimal non-null separators of $M$ as its elementary separators. From the foregoing observations we deduce

3.41 The elementary separators of $M$ are disjoint nonnull subsets of $E$ whose union is $E$.

3.42 Suppose $S \subseteq E$. Then $S$ is a separator of $M$ if and only if

$$
M \cdot S=M \times S .
$$

Proof: Let $S$ be a separator of $M$. Then an atom of $M$ has a non-null intersection with $S$ if and only if it is itself a subset of $S$. It follows that $M \times S$ and $M \cdot S$ are identical.

Conversely suppose $M \cdot S=M \times S$, and let $Y$ be any atom of $M$. If it meets $S$ it contains an atom of $M \cdot S$, that is $M \times S$. It is then itself an atom of $M \times S$ and $M$, by Axiom I. Thus $Y \subseteq S$. We deduce that $S$ is a separator of $M$.

3.43 Let $S$ be a separator of $M$. Then, for each $T \subseteq E$, $S \cap T$ is a separator of both $M \cdot T$ and $M \times T$. 
Proof: Let $Y$ be an atom of $M \cdot T$ or $M \times T$. There is an atom $Z$ of $M$ such that $Y=Z \cap T$. Either $Z \subseteq S$ or $Z \subset E-S$. Hence $Y$ is contained either in $S \cap T$ or in $(E-S) \cap T$. The theorem follows.

3.44 Let $S$ be a separator of $M$, and let $T$ be a separator of $M \cdot S$, that is $M \times S$ by 3.42 . Then $T$ is a separator of $M$.

Proof: $M \times T=(M \times S) \times T=(M \times S) \cdot T=(M \cdot S)$ $\cdot T=M \cdot T$, by $3.331,3.332$ and 3.42 . Hence $T$ is a separator of $M$, by 3.42 .

3.45 The separators of $M^{*}$ are the separators of $M$.

Proof: If $M \cdot S=M \times S$ we have $M^{*} \times S=M^{*} \cdot S$, by 3.351 and 3.352 . Similarly if $M^{*} \cdot S=M^{*} \times S$ we have $M \times S=M \cdot S$, by $3.351,3.352$, and 2.65 . The theorem follows, by 3.42 .

If $S$ is an elementary separator of $M$ we refer to the matroid $M \cdot S$, that is $M \times S$, as a component of $M$.

The matroid $M$ is said to be connected if it has no separators other than the trivial ones, $E$ and its null subset. Thus $M$ is connected if and only if either $E$ is null or $M$ has just one elementary separator.

3.46 If a minor $(M \cdot S) \times T$ of $M$ is connected, and $T$ is not null, then $T$ is a subset of an elementary separator of $M$.

We obtain this result by two applications of 3.43 . 3.47 Any component of $M$ is connected (by 3.44). 3.48 Let $M \times S$ and $M \times T$ be connected reductions of $M$ such that $S \cap T$ is non-null. Then $M \times(S \cup T)$ is connected.

Proof: There is an elementary separator $Z$ of $M$ $\times(S \cup T)$ which meets $S \cap T$, by 3.41. It contains both $S$ and $T$, by 3.331 and 3.43. Hence $Z=S \cup T$, and the theorem follows.

3.49 The components of $M^{*}$ are the duals of the components of $M$.

This follows from 3.45, with the help of 3.351, 3.352 and 3.42 .

Consider a graph $G$ without isolated vertices. It is called nonseparable or cyclically connected if it has the following property: for any two complementary non-null subsets $S$ and $T$ of $E(G)$ there exists a polygon of $G$ whose edge-set meets both $S$ and $T$.

The property of cyclic connection in graphs corresponds to that of connection in matroids. For it is clear that $G$ is nonseparable if and only if $P(G)$ is connected. Hence $G$ is nonseparable if and only if $B(G)$ is connected, by 2.67 and 3.45 .

There is no property of matroids in general which corresponds in this way to ordinary connection for graphs.

\subsection{Properties of Rank}

Let $M$ be a matroid on a set $E$. From the definition of rank given in section 2.2 we have the following theorem.

$3.510 \leqslant r(M) \leqslant|E|$. Moreover $r(M)=0$ if and only if $M$ has no atom, and $r(M)=|E|$ if and only if each cell of $E$ constitutes by itself an atom of $M$.

From 2.64 we deduce

$$
r(M)+r\left(M^{*}\right)=|E| .
$$

We now supplement these results with some formulae involving the ranks of minors of $M$.

3.53 Let $S$ be any subset of $E$. Let $C$ be a dendroid of $M \times S$ and let $D$ be a dendroid of $M \cdot(E-S)$. Then $C \cup D$ is a dendroid of $M$.

Proof: Let $a$ be any cell of $C \cup D$.

If $a \epsilon C$ we define $F_{a}$ as the atom $J(C, a)$ of $M \times S$. It is of course also an atom of $M$, and $\left|F_{a} \cap D\right|=0$.

If $a \in D$ we define $F_{a}$ as an atom of $M$ for which $F_{a} \cap D=\{a\}$ and for which $\left|F_{a} \cap C\right|$ has the least value consistent with this condition. Such a set exists since the atom $J(D, a)$ of $M \cdot(E-S)$ is the intersection with $E-S$ of some atom of $M$. We show that in fact $\left|F_{a} \cap C\right|=0$. For suppose $b \epsilon F_{a} \cap C$. Applying Axiom II to $F_{a}$ and the atom $Y=J(C, b)$ of $M \times S$ we find that there is an atom $F$ of $M$ such that $a \epsilon F \subseteq\left(F_{a} \cup Y\right)-\{b\}$. But then $F \cap D=\{a\}$ and $|F \cap C|<\left|F_{a} \cap C\right|$, which is contrary to the definition of $F_{a}$.

We conclude that for each $a \epsilon C \cup D$ there is an atom $F_{a}$ of $M$ such that $F_{a} \cap(C \cup D)=\{a\}$.

On the other hand any atom of $M$ meets $D$ if it has a non-null intersection with $E-S$, and meets $C$ if it is contained in $S$. Hence $C \cup D$ is a dendroid of $M$.

We note the following corollaries.

$$
r(M \times S)+r(M \cdot(E-S))=r(M) .
$$

3.55 Suppose $a \epsilon E$. Then $r(M)-r(M \times(E-\{a\}))=1$ or 0 according as a does or does not belong to some atom of $M$.

The next theorem finds frequent applications in the next chapter.

\subsection{Let $S$ and $T$ be subsets of $E$. Then}

$r(M \times(S \cup T))+r(M \times(S \cap T)) \geqslant r(M \times S)+r(M \times T)$.

Proof: Let $C$ be a dendroid of $M \times(S \cap T)$. Let $D_{1}$ be a dendroid of $(M \times S) \cdot(S-T)$ and $D_{2}$ a dendroid of $(M \times T) \cdot(T-S)$. Write $U=C \cup D_{1} \cup D_{2}$.

Now $C \cup D_{1}$ is a dendroid of $M \times S$, by 3.331 and 3.53. Hence if $a \epsilon C \cup D_{1}$ there is an atom $F_{a}$ of $M$ $\times(S \cup T)$, contained in $S$, which meets $U$ only in the cell $a$. An analogous result holds for $a \epsilon C \cup D_{2}$. We deduce that each cell of $(M \times(S \cup T)) \cdot U$ constitutes an atom of that matroid. Accordingly

$$
\begin{aligned}
r((M \times(S \cup T)) \cdot U) & =\left|C \cup D_{1} \cup D_{2}\right|, \\
& =r(M \times S)+r(M \times T)-r(M \times(S \cap T)) .
\end{aligned}
$$

The theorem follows, by 3.54 .

$3.57 r(M)=1$ if and only if $M$ has just one atom.

Proof: If $M$ has just one atom $X$, then any cell of $X$ constitutes a dendroid of $M$, and $r(M)=1$. Conversely if $r(M)=1$ then $M$ has a dendroid $D$ consisting of a single cell $a$. But $M$ has only one atom $J(D, a)$ which includes $a$. 


\section{Geometry of Matroids}

\subsection{Flats}

Let $M$ be a matroid on $a$ set $E$. We propose to study the system of contractions $M \times S$ of $M$. We abbreviate the expression $r(M \times S)$ as $r S$. For the sake of geometrical analogies to be explained below we define the dimension $d S$ of $S$ as the number $r S-1$.

A subset $S$ of $E$ is called a flat of $M$ if it is a union of atoms of $M$. The null subset of $E$ is counted as a null union of atoms, and therefore a flat. For any subset $S$ of $E$ there is an associated flat $\langle S\rangle$, defined as the union of all the atoms of $M$ contained in $S$, that is the union of the atoms of $M \times S$. By 3.54 we have

\subsection{1}

$$
d\langle Z\rangle=d Z=r Z-1 .
$$

In what follows we are concerned with the lattice of flats of $M$, as partially ordered by the inclusion relation.

4.12 The only flat of $M$ with negative dimension is the null flat $\phi$, for which $d \phi=-1$. The flats of dimension 0 are the atoms of $M$.

This follows from 3.51 and 3.57. It is convenient to take over some more geometrical terminology at this stage. We say that the flat $S$ is on the flat $T$ if either $S \subseteq T$ or $T \subseteq S$. The atoms of $M$ are called also its points, and flats of dimensions 1 and 2 are lines and planes of $M$ respectively. More generally a flat of dimension $k$ is called a $k$-flat.

4.13 If $S$ is a flat of $M$ and $a \epsilon S$, then

$$
d\langle S-\{a\}\rangle=d S-1,
$$

by 3.55 .

4.14 If $S$ and $T$ are flats of $M$ such that $S \subset T$, then $d S<d T$. Moreover there is a flat $U$ of $M$ such that $S \subset U \subseteq T$ and $d U=d S+1$.

Proof: The first statement follows from 3.54. To prove the second we define $U$ as a flat of $M$ satisfying $S \subset U \subseteq T$ and such that $|U|$ has the least value consistent with this condition. Choose $a \epsilon U-S$. Then $S \subseteq\langle U-\{a\}\rangle \subset T$, and therefore $S=\langle U-\{a\}\rangle$ by the choice of $U$. Hence $d U=d S+1$, by 4.13 .

4.15 Let $S$ and $T$ be flats of $M$ such that $S \subseteq T$. Then there exists a flat $U$ of $M$ such that $U \subseteq T,\langle\bar{U} \cap S\rangle=\phi$ and $d U=d T-d S-1$.

Proof: Write $S_{0}=S$ and $T_{0}=T$. If possible choose $a_{0} \epsilon S_{0}$ and write $S_{1}=\left\langle S_{0}-\left\{a_{0}\right\}\right\rangle, T_{1}=\left\langle T_{0}-\left\{a_{0}\right\}\right\rangle$. It is clear that $S_{1} \subseteq T_{1}$. If possible choose $a_{1} \epsilon S_{1}$ and write $S_{2}=\left\langle S_{1}-\left\{a_{1}\right\}\right\rangle, T_{2}=\left\langle T_{1}-\left\{a_{1}\right\}\right\rangle$. Then $S_{2} \subseteq T_{2}$.

Continue this process until it terminates. By 4.13 this will be with $S_{k}$ and $T_{k}$, where $k=d S+1$ and $S_{k}=\phi$. Applying 4.13 to the sequence of the $T_{i}$ we find that $d T_{k}=d T-d S-1$. But $\left\langle T_{k} \cap S\right\rangle=\left\langle S-\left\{a_{0}, a_{1}, \ldots\right.\right.$. $\left.\left.a_{k-1}\right\}\right\rangle=S_{k}=\phi$. Hence the theorem is satisfied with $U=T_{k}$.

4.16 Let $S$ and $T$ be flats of $M$. Then

$$
d(S \cup T)+d\langle S \cap T\rangle \geqslant d S+d T,
$$

by 3.56 .
Many "geometrical" results can be deduced from 4.14 and 4.16. An example follows.

4.171 Let $\mathrm{L}_{1}$ and $\mathrm{L}_{2}$ be distinct lines of $M$ on the same plane $P$. Then $\mathrm{L}_{1}$ and $\mathrm{L}_{2}$ have just one common point.

Proof: We have $L_{1} \subseteq L_{1} \cup L_{2} \subseteq P$. Hence $L_{1} \cup L_{2}$ $=P$, by 4.14. Another application of 4.14 shows that $d\left\langle L_{1} \cap L_{2}\right\rangle \leqslant 0$ since $L_{1} \cap L_{2} \subset L_{1}$. But $d\left\langle L_{1} \cap L_{2}\right\rangle \geqslant 0$ by 4.16. Hence $d\left\langle L_{1} \cap L_{2}\right\rangle=0$, and the theorem follows by 4.12 .

The two following theorems can be proved in a similar way.

4.172 Let $P_{1}$ and $P_{2}$ be distinct planes on the same 3flat $F$ of $M$. Then $\left\langle P_{1} \cap P_{2}\right\rangle$ is a line on $F$.

4.173 Let $P$ be a plane and $L$ a line on the same 3flat $F$. Suppose $L$ is not on $P$. Then $\langle P \cap L\rangle$ is a point on $E$.

We observe that if $G$ is any graph, then the flats of $P(G)$ correspond to those subgraphs of $G$ which are unions of polygons.

\subsection{Connected Flats}

A flat $S$ of $M$ is called "connected" if $M \times S$ is a connected matroid. We then refer to the separators of $M \times S$ as the "separators of $S$ ". The lattice of connected flats of $M$ has some very interesting properties. We begin this section with a study of the line.

4.21 Let $L$ be a line of $M$, and suppose $a \epsilon L$. Then $\langle L-\{a\}\rangle$ is the only point on $L$ which does not include $a$.

Proof: $\langle L-\{a\}\rangle$ is a point on $L$, by 4.13 . But if $X$ is any point on $L$ not including $a$ we have $X \subseteq$ $\langle L-\{a\}\rangle$ and therefore $X=\langle L-\{a\}\rangle$ by Axiom I.

4.22 Any line $L$ of $M$ is on two distinct points. If $X$ and $Y$ are distinct points on $L$ then $L=X \cup Y$. Moreover $X \cap Y$ is non-null if and only if $L$ is connected.

Proof: Choose $a \epsilon L$ and $b \epsilon\langle L-\{a\}\rangle$. Then $\langle L-\{a\}\rangle$ and $\langle L-\{b\}\rangle$ are distinct points of $L$.

Let $X$ and $Y$ be distinct points on $L$. Then $X \subset$ $X \cup Y \subseteq L$. Hence $X \cup Y=L$, by 4.14. If $X \cap Y$ is nonnull then $L$ is connected, by 3.48. If $X \cap Y$ is null then either $X$ and $Y$ are nontrivial separators of $L$, or there is a point $Z$ on $L$ meeting both $X$ and $Y$. In the latter case $X \subset X \cup Z \subset X \cup Y=L$, by Axiom I. But this is impossible, by 4.14.

$4.23 \mathrm{~A}$ disconnected line is on just two points, and a connected line is on at least three points.

Proof: By 4.22 any two distinct points on a disconnected line $L$ are disjoint, and have $L$ as their union. Hence $L$ has at most two points, and therefore just two, by 4.22 .

By 4.22 any connected line $L$ has two distinct points $X$ and $Y$, and we can find $a \in X \cap Y$. By 4.21 $\langle L-\{a\}\rangle$ is a point on $L$ which is distinct from $X$ and $Y$.

It should perhaps be pointed out that two distinct points $X$ and $Y$ of a matroid $M$ are not necessarily on a common line. There is indeed a flat $X \cup Y$, but its dimension may exceed 1 . However the following theorem is sometimes helpful.

4.24 Let $T$ be a separator of a flat $S$ of $M$. Let $X$ and $Y$ be points on $S$ such that $X \subseteq T$ and $Y \subseteq S-T$. Then $X \cup Y$ is a disconnected line of $M$. 
Proof: $X$ and $Y$ are separators of the flat $X \cup Y$, by 3.43. Hence $d(X \cup Y)=d X+d Y-1=1$, by 3.42 and 3.54. Thus $X \cup Y$ is a disconnected line.

We go on to prove two important theorems, 4.26 and 4.27, about connected flats in general. We need the auxiliary result

4.25 Let $S$ and $T$ be connected flats of $M$ such that $S \subset T$. Then there exists a connected $(d S+1)-$ flat $U$ of $M$ such that $\mathrm{S} \subset \mathrm{U} \subseteq \mathrm{T}$.

Proof: Since $T$ is connected we can find a point $X$ of $M$ such that $X \subseteq T$ and $X$ meets both $S$ and $T-S$. Choose such an $X$ so that $|S \cup X|$ has the least possible value.

$S \cup X$ is a connected flat of $M$, by 3.48. Its dimension exceeds $d S$, by 4.14 .

Suppose $d(S \cup X)>d S+1$. Choose $a \in X-S$. Then

$$
d\langle(S \cup X)-\{a\}\rangle \geqslant d S+1,
$$

by 4.13. Hence there is a point $Y$ of $M$ such that $Y \subseteq(S \cup X)-\{a\}$ and $Y$ meets $X-S$. But $Y \cap S=\phi$ by the choice of $X$. Hence $Y \subset X$, which is contrary to Axiom I. We deduce that in fact $d(S \cup X)=d S+1$. Hence the theorem holds with $U=S \cup X$.

4.26 Let $S$ be a connected d-flat on a connected $(d+2)$ flat $T$ of $M$. Then there exist distinct connected $(d+1)$ flats $U$ and $V$ of $M$ such that $S=\langle U \cap V\rangle$ and $T=U \cup V$.

Proof: The following argument is illustrated by figure $4 \mathrm{~A}$ for the case $d=0$, in which $S$ is a point and $T$ is a connected plane.

By 4.25 there is a connected $(d+1)$-flat $U$ which is on both $S$ and $T$. Choose $a \epsilon U-S$ and write $W$ $=\langle T-\{a\}\rangle$. By $4.13 W$ is another $(d+1)$-flat on both $S$ and $T$. By 4.15 there is a line $L$ on $T$ having no common point with $S$. It meets $U$ and $W$ in points $X$ and $Z$, respectively, by 4.16 . By 4.14 we have $S \cup X=U$ and $S \cup Z=W$. Hence $Z$ is not on $U$ and $U \cup Z=T$, by further applications of 4.14.

Assume $W$ is not connected. Then $S \cap Z=\phi$, by 3.48 .

Suppose $U \cap Z=\phi$. By the connection of $T$, that is $U \cup Z$, there is an atom $Z^{\prime}$ of $M$ such that $Z^{\prime} \subseteq T$ and $Z^{\prime}$ meets both $U$ and $Z$. Then $U \subset U \cup Z^{\prime} \subset U \cup Z$ $=T$, by Axiom I. But this is impossible by 4.14. We deduce that $U \cap Z$ is non-null. A similar argument in which $X, S$, and $U$ replace $Z, U$, and $T$ respectively shows that $X \cap S$ is not null. Choose $b \in Z \cap U$ and $c \epsilon X \cap S$.

Write $V=\langle T-\{b\}\rangle$. By $4.13 V$ is a $(d+1)$-flat. It is on $S$, since $b \in Z$ and $S \cap Z=\phi$. By 4.16 it has a common point $Y$ with $L$. Since $L$ and $S$ have no common point we deduce from 4.14 that $V=S \cup Y$. Moreover $V$ is distinct from $U$ and $W$, since $b \in Z \cap U \subseteq W \cap U$.

Now $c \in S \cap X \subseteq S \cap L=S \cap(Y \cup Z)$, by $4.22, \stackrel{=}{=} S \cap Y$, by our assumption. Hence $V$ is connected, by 3.48 .

In the remaining case, in which $W$ is connected, we write $V=W$.

In either case we have two distinct connected $(d+1)$-flats $U$ and $V$ of $M$, each of which is on both $S$ and $T$. Hence

$$
S \subseteq\langle U \cap V\rangle \subset U \subset U \cup V \subseteq T,
$$

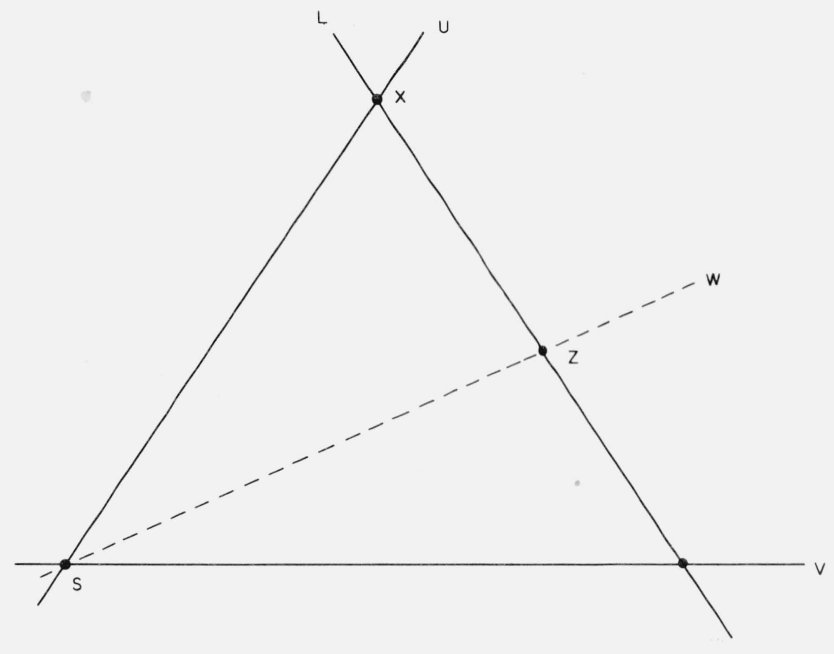

Figure 4A

since neither of $U$ or $V$ contains the other, by 4.14 . Another application of 4.14 shows that $S=\langle U \cap V\rangle$ and $T=U \cup V$.

4.27 Let $S, T$, and $U$ be flats of $M$ such that $S$ and $T$ are connected, $S \cup U \subseteq T$ and $\langle S \cap U\rangle=\phi$. Then there exists a connected flat $R$ of $M$ such that $S \subseteq R \subseteq T$, $\langle R \cap U\rangle=\phi$, and $d R=d T-d U-1$.

Proof: If possible choose $S, T$, and $U$ so that the theorem fails and $d U$ has the least value consistent with this condition. Then $d U>-1$, since otherwise the theorem holds with $T=R$.

Let $W$ be a connected flat of $M$ of greatest possible dimension such that $S \subset W \subset T$ and $W$ does not contain $U$. Suppose $d W<d T-1$. Then, by 4.26 , there are distinct connected $(d W+1)$-flats $K$ and $L$ on $T$ such that $\langle K \cap L\rangle=W$. But then $K$ and $L$ cannot both contain $U$, and this is contrary to the choice of $W$. We deduce, using 4.14 , that $d W=d T-1$. We note that $d\langle U \cap W\rangle<d U$, by 4.14 .

By the choice of $S, T$, and $U$ there is a connected flat $R$ of $M$ such that $S \subseteq R \subseteq W \subset T,\langle R \cap\langle U \cap W\rangle\rangle$ $=\phi \quad$ and $\quad d R=d W-d\langle U \cap W\rangle-1$. But then $\langle R \cap U\rangle=\phi \quad$ and $\quad d R \geqslant d T-d U-1$. However $d R \leqslant d T-d U-1$, by 4.14 and 4.16 . We deduce that $d R=d T-d U-1$. Thus the theorem holds for $S, T$, and $U$, which is a contradiction.

It follows that the theorem is true in general.

We shall also need the following more special theorem.

4.28 Let $L$ be a disconnected line on a connected d-flat $S$ of $M$, where $d S>1$. Then there exists a connected plane $P$ of $M$ such that $L \subset P \subseteq S$.

Proof: Let the two points on $L$ be $X$ and $Y$. (See 4.23.) Let $P$ be a connected flat of $M$, of least possible dimension, such that $L \subset P \subseteq S$. Assume $d P>2$.

Suppose first that there is a second disconnected line $L^{\prime}$ on $X$ and $P$. Let its point other than $X$ be $Z$. Then $X, Y$, and $Z$ are distinct points, by 4.14. By 4.27 there is a connected $(d P-2)$-flat $U$ on $Y$ and $P$ which has no point in common with $L^{\prime}$. By 4.26 
there are distinct connected $(d P-1)$-flats $V$ and $W$ on $P$ such that $\langle V \cap W\rangle=U$. These two flats meet $L^{\prime}$ in distinct points, by 4.14. Since there are only two points on $L^{\prime}$ we may suppose $X$ is on $V$. But then $L$ is on $V$ and the definition of $P$ is contradicted.

We deduce that there is no second disconnected line on $X$ and $P$, and similarly no second disconnected line on $Y$ and $P$.

Choose $a \in P-L$ and write $R=\langle P-\{a\}\rangle$. Then $L \subseteq R$, and $d R=d P-1$ by 4.13 . By the definition of $P$ the flat $R$ is disconnected. But the only possible non-trivial separators of $R$ are $X$ and $Y$, by 4.24. Accordingly $R=L$ and $d P=2$, contrary to assumption. The theorem follows.

4.281 Let $L$ be a disconnected line on a plane $P$ of $M$. Let $X$ and $Y$ be its two points, and let $Z$ be any other point on $P$. Then $X \cup Z$ and $Y \cup Z$ are connected lines, the only lines of $M$ which are on both $Z$ and $P$.

Proof: Any line on $P$ contains $X$ or $Y$, by 4.171 . Hence, by 4.22 , the only flats on $Z$ and $P$ which can be lines are $X \cup Z$ and $Y \cup Z$. They must in fact be connected lines, by 4.26 .

4.282 Let $L$ be a disconnected line on a connected plane $P$ of $M$. Then every line on $P$ other than $L$ is connected.

Proof: Let $L^{\prime}$ be such a line. It is on some point $Z$ not contained in $L$, by 4.22 . Hence it is connected, by 4.281 .

The foregoing results can be applied to the polygonmatroid of a graph $G$ to obtain some rather simple results in graph theory. A set $S$ is a connected flat of $P(G)$ if and only if $G \cdot S$, whose polygon matroid is $P(G) \times S$ by 3.371 , is nonseparable. The rank of $P(G)$ is the cyclomatic number of $G$, that is the least number $p_{1}(G)$ of edges of $G$ which must be deleted in order to destroy every polygon.

Two connected flats $S$ and $T$ of $P(G)$ such that $\langle S \cap T\rangle=\phi$ correspond to two nonseparable subgraphs of $G$ which have no common polygon.

\subsection{Linear Subclasses}

Let $M$ be a matroid on a set $E$, and let $C$ be any class of points of $M$. We call $C$ a linear subclass of $M$ if it has the following property. If two distinct points $X$ and $Y$ of $C$ are on a common line $L$ in $M$, then every point on $L$ belongs to $C$. In earlier work on matroids a linear subclass is called a "convex subclass".

An obvious example of a linear subclass of $M$ is provided by the class of all points on a given flat.

Another important example arises as follows. Let us say that a subset $S$ of $E$ cuts another subset $T$ of $E$ if both $S \cap T$ and $T-S$ are non-null. Then we have the following theorem.

4.31 Let $S$ be any subset of $E$. Let $C$ be the class of all atoms of $M$ which do not cut $S$. Then $C$ is a linear subclass of $M$.

Proof: Let $X$ and $Y$ be points of $C$ on a common line $L$. Let $Z$ be any point on $L$. We have $Z \subset X \cup Y$ $=L$, by 4.22 .

If $X \cap S$ and $Y \cap S$ are null we have $Z \cap S=\phi$ and therefore $Z \epsilon C$. If $S \subseteq X$ and $S \subseteq Y$ we have $S \subseteq Z$ and therefore $Z \epsilon C$.

In the remaining case we may suppose $X \cap S=\phi$ and $S \subseteq Y$. Hence $L$ is disconnected, by 4.22 . Accordingly $Z=X$ or $Y$, by 4.23 . Again we have $Z \epsilon C$.

The theorem follows.

We have so far presented the theory of matroids as something more general than that of graphs, having its own theorems from which the results of graph theory can be derived as special cases. It is somewhat surprising therefore to find that we must now use the technique of graph theory in order to study linear subclasses. However each matroid does have an associated graph. The vertices are the points of the matroid, and two points are said to be adjacent if and only if they are distinct points of the same connected line.

A path in $M$ is a finite sequence

$$
P=\left(X_{1}, \ldots, X_{k}\right)
$$

of one or more points of $M$, not necessarily all distinct, such that any two consecutive terms are adjacent (and therefore distinct) points of $M$. We refer to $X_{1}$ and $X_{k}$ as the origin and terminus of $P$ respectively. If they coincide we call $P$ re-entrant. If $P$ has only one term it is degenerate. The length $s(P)$ of $P$ is one less that the number of terms of $P$.

If $P=\left(X_{1}, \ldots, X_{k}\right)$ and $Q=\left(X_{k}, \ldots, X_{m}\right)$ are paths of $M$ such that the origin of $Q$ is the terminus of $P$, then we define their product $P Q$ as the path $\left(X_{1}, \ldots\right.$, $\left.X_{k}, \ldots, X_{m}\right)$. Multiplication of paths is clearly associative. We may therefore write a product $(P Q) R$ or $P(Q R)$ simply as $P Q R$

If $P$ is any path of $M$ we write $P^{-1}$ for the path obtained by taking the terms of $P$ in reverse order. The following rules are obvious.

$$
\left(P^{-1}\right)^{-1}=P
$$

$$
(P Q)^{-1}=Q^{-1} P^{-1} \text {. }
$$

If every term of a path $P$ is on a flat $S$ we say that $P$ is on $S$.

A path $P$ in which the terms are all distinct is called simple. As an example of a theorem on paths we offer the following.

4.33 Let $P$ be a path from $X$ to $Y$ on a flat $S$ of $M$. Then there is a simple path from $X$ to $Y$ on $S$.

Proof: Let $P_{1}$ be a shortest path from $X$ to $Y$ on $S$. If it is not simple we can write it as a product $Q R T$ where $R$ is re-entrant and nondegenerate. But then $Q T$ is a path from $X$ to $Y$ on $S$ which is shorter than $P_{1}$. We conclude that in fact $P_{1}$ is simple.

The fundamental theorem about linear subclasses runs as follows.

4.34 Let $C$ be a linear subclass of $M$. Let $S$ be a connected flat of $M$, and let $X$ and $Y$ be points on $S$ such that $Y$ is not in $C$. Then there is a simple path $P$ from $X$ to $Y$ on $S$ such that no term of $P$ other than $X$ belongs to $C$. 
Proof: If possible choose $S, X$ and $Y$ so that the theorem fails and $d S$ has the least value consistent with this. Clearly $d S>1$ and $Y$ is distinct from $X$. Figure $4 \mathrm{~B}$ illustrates the following argument for the case $d S=3$.

By 4.25 and 4.26 there is a connected $(d S-2)$-flat $U$ and two connected $(d S-1)$-flats $V$ and $W$ such that $X \subseteq U=\langle V \cap W\rangle$ and $V \cup W=S$. Moreover $Y$ is not on $V$ or $W$, by the choice of $S, X$, and $Y$.

By 4.27 there is a line $L$ on $S$ such that $\langle L \cap U\rangle=\phi$. It meets $V$ and $W$ in distinct points $T$ and $Z$, respectively. They are not both in $C$, for otherwise $Y$ would be in $C$. Without loss of generality we may suppose $T$ not in $C$,

By the choice of $S, X$, and $Y$ there is a simple path $Q$ from $X$ to $T$ on $V$ such that no term of $Q$ other than the first is a point on $C$. The product $Q(T, Y)$ may be taken as the required simple path $P$. As this result is contrary to the choice of $S, X$, and $T$ the theorem follows.

A slight modification of the above proof shows that we can arrange that $s(P)=d S$.

The following theorem is analogous to a well-known result in graph theory.

4.35 Let $S$ be any flat of $M$. Then $S$ is connected if and only if for any two points $X$ and $Y$ on $S$ there exists a simple path on $S$ from $X$ to $Y$.

Proof: Suppose $S$ is connected. Let $X$ and $Y$ be points of $S$. Then there is a simple path on $S$ from $X$ to $Y$, by 4.34 , with $C=\phi$.

Now suppose $S$ has a nontrivial separator $T$. There are points $X$ and $Y$ on $S$ such that $X \subseteq T$ and $Y \subseteq S-T$. If there is a path on $S$ from $X$ to $Y$ there are consecutive terms $X^{\prime}$ and $Y^{\prime}$ of $P$ such that $X^{\prime} \subseteq T$ and $Y^{\prime} \subseteq S-T$. But this is impossible since $X^{\prime} \cup Y^{\prime}$ is not a connected line, by 4.24 .

4.36 Let $C$ be any linear subclass of $M$. Let $S$ be a $d$-flat of $M$ on a $(d+1)$-flat $T$. Suppose all the points on $S$ and at least one other point on $T$ belong to $C$. Then all the points on $T$ belong to $C$.

Proof: Suppose the theorem fails. Then we can find points $X \epsilon C$ and $Y \notin C$, each being on $T$ but not on $S$. The flat $X \cup Y$ is connected since otherwise $S \subset S \cup X \subset T$, contrary to 4.14 .

By 4.34 there is a path from $X$ to $Y$ on $X \cup Y$ whose second term, $X^{\prime}$ say, is not in $C$. By 4.16 the connected line $X \cup X^{\prime}$ has a point $X^{\prime \prime}$ in common with $S$. But $X^{\prime \prime}$ is not in $\mathrm{C}$, by the definition of a linear subclass. This is contrary to hypothesis.

4.37 Let $S$ be a connected flat of $M$. Let $C_{1}$ and $C_{2}$ be linear subclasses of $M$, neither of which includes all the points on $S$. Then there is a point $Z$ on $S$ which belongs to neither $C_{1}$ nor $C_{2}$.

Proof: Choose points $X$ and $Y$ on $S$ such that $X \notin C_{1}$ and $Y \xi C_{2}$. We may suppose $X$ and $Y$ distinct since otherwise the theorem is trivially true.

By 4.34 there is a point $X^{\prime}$ on $S$ which is adjacent to $X$ and not in $C_{2}$. We may suppose $X \epsilon C_{2}$ and $X^{\prime} \epsilon C_{1}$, for otherwise one of these points could be taken as $Z$. But now there is a third point $Z$ on the connected line $X \cup X^{\prime}$, and this can belong neither to $C_{1}$ nor to $C_{2}$.

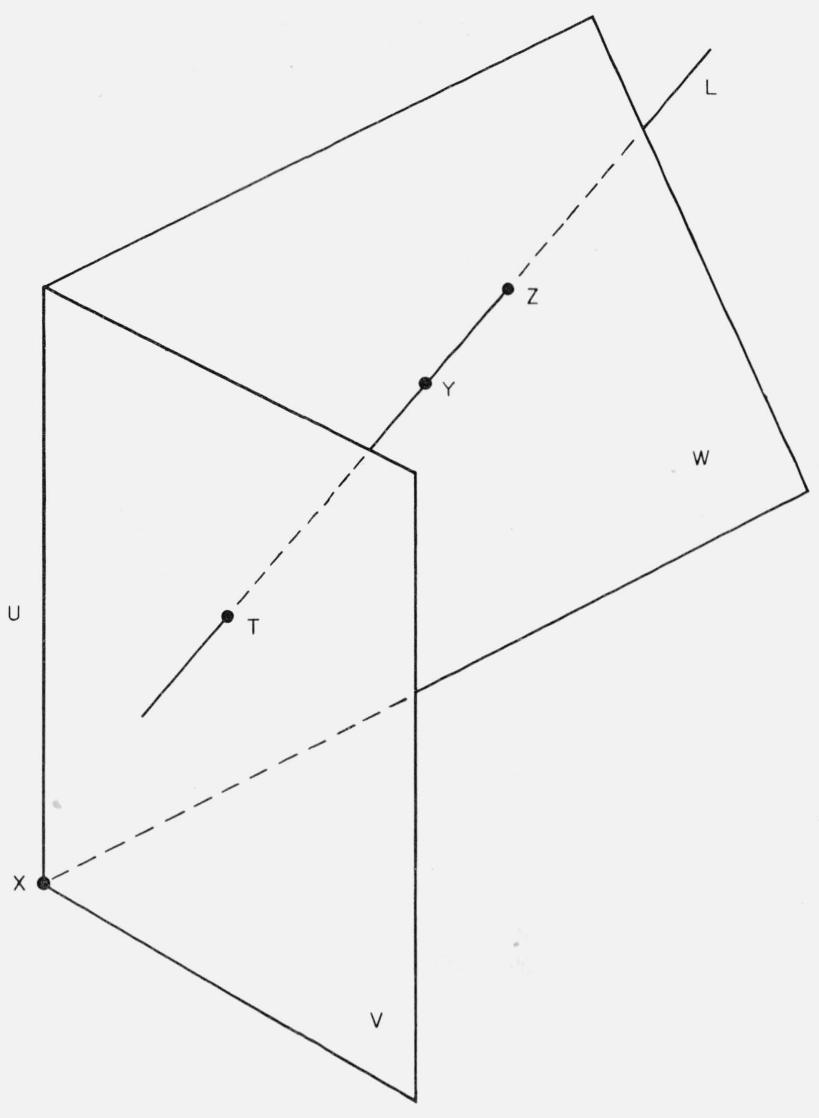

Figure 4B

As important special cases of 4.37 we note the following.

4.371 Let $a$ and $b$ be distinct cells of a connected matroid $M$. Then there is an atom of $M$ which includes both $a$ and $b$.

4.372 Let $S$ and $T$ be subsets of $E$, each cut by some atom of $M, M$ being connected. Then some atom of $M$ cuts both $S$ and $T$.

To prove 4.371 we take $C_{1}$ to be the set of points on $\langle E-\{a\}\rangle$, and $C_{22}$ to be the set on $\langle E-\{b\}\rangle .4 .372$ follows from 4.31 .

When we apply 4.371 to the polygon-matroid of a graph we find that if $a$ and $b$ are distinct edges of a nonseparable graph $G$, then there is a polygon of $G$ through both $a$ and $b$. This is one of the theorems of Hassler Whitney on graphs.

\subsection{Carriers}

Let $M$ be a matroid on a set $E$, and $S$ be any subset of $E$. We proceed to discuss the relations between the flats of $M \cdot S$ and those of $M$. Dimensions referring to $M \cdot S$ will be distinguished in formulae by primes.

A carrier of $M \cdot S$ in $M$ is a subset $Z$ of $E$ such that

$$
(M \times Z) \cdot S=M \cdot S
$$


and $d\langle Z\rangle$ has the least value consistent with this property. Such a carrier exists, since $(M \times E) \cdot S$ $=M \cdot S$

4.41 Let $Z$ be a carrier of $M \cdot S$ in $M . \quad$ Then $\mathrm{dZ}=\mathrm{d}^{\prime} \mathrm{S}$.

Proof: Assume there is an atom $X$ of $M$ such that $X \subseteq Z-S$. Choose $a \in X$ and write

$$
M_{1}=(M \times(Z-\{a\})) \cdot S .
$$

Let $Y$ be an atom of $M_{1}$. Then there is an atom $U$ of $M$ such that $U \subseteq Z-\{a\}$ and $Y=S \cap U$. Hence there is an atom $Y^{\prime}$ of $M \cdot S$ such that $Y^{\prime} \subseteq Y$.

Conversely let $Y^{\prime}$ be an atom of $M \cdot \bar{S}=(M \times Z) \cdot S$. There is an atom $V$ of $M \times Z$ such that $Y^{\prime}=S \cap V$. Suppose $a \epsilon V$. Then by applying Axiom II to $V$ and $X$ we find that there is an atom $U$ of $M \times Z$ such that $S \cap U$ is a non-null subset of $Y^{\prime}$, and $a \notin U$. If $a \notin V$ we write $U=V$. In each case $U$ is an atom of $M \times(Z$ - $\{\mathrm{a}\})$ and $S \cap U$ is non-null. Hence there exists an atom $Y$ of $M_{1}$ such that $Y \subset S \cap U \subset Y^{\prime}$.

Applying Axiom I to the foregoing results we find that $M_{1}=M \cdot S$. But this is contrary to the definition of $Z$ as a carrier of $M \cdot S$. We deduce that $d(Z-S)$ $=-1$. The theorem now follows from 3.54 .

4.42 Let $Z$ be a carrier of $M \cdot S$ in $M$. Let $X$ be an atom of $M \cdot S$. Then there is just one atom $Y$ of $M \times Z$ such that $S \cap Y=X$.

Proof: Such an atom $Y$ exists, since $M \cdot S=(M \times Z) \cdot S$. Suppose $Y^{\prime}$ is another such atom of $M \times Z$. Choose $a \epsilon S \cap Y=S \cap Y^{\prime}=X$. By Axiom I we can find $b \in Y^{\prime}-Y$. By Axiom II there is an atom $U$ of $M \times Z$ such that $b \epsilon U \subseteq\left(Y \cup Y^{\prime}\right)-\{a\}$.

We have $S \cap U \subset S \cap Y=X$. Hence $S \cap U$ is null. since $M \cdot S$ satisfies Axiom I. Hence $U \subseteq Z-S$. But then $d\langle Z-S\rangle>-1$, and $d Z>d^{\prime} S$, by 3.54 . This is contrary to 4.41 .

We can now define a mapping $\theta$ of the set of flats of $M \cdot S$ onto a class of flats of $M \times Z$, in the following way

(i) If $T$ is the null flat of $M \cdot S$ then $\theta T$ is the null flat of $M \times Z$.

(ii) If $T$ is an atom of $M \cdot S$, then $\theta T$ is the unique atom $U$ of $M$ on $Z$ such that $S \cap U=T$. (4.42).

(iii) If $T$ is a $d^{\prime}$-flat of $M \cdot S$, where $d^{\prime} \geqslant 1$, then $\theta T$ is the union of all atoms $\theta X$ of $M \times Z$ such that $X$ is an atom of $M \cdot S$ on $T$.

4.43 If $T$ and $U$ are flats of $M \cdot S$, then $\theta T \subseteq \theta U$ if and only if $T \subseteq U$.

This follows at once from the definition of $\theta$. It implies that $\theta T$ and $\theta U$ are distinct if $T$ and $U$ are distinct, and that $\theta T \subset \theta U$ if and only if $T \subset U$. 4.44 If $T$ is a flat of $M \cdot S$, then $d^{\prime} T=d \theta T$.

Proof: By 4.14 we can construct a sequence

$$
\left.T_{-1}, T_{0}, T_{1}, \ldots ., T_{d^{\prime}}\right)
$$

of flats of $M \cdot S$, where $d^{\prime}=d^{\prime} S$, such that each member of the sequence except $T_{d^{\prime}}$ is a proper subset of its successor, such that $d^{\prime} T_{i}=i$ for each $T_{i}$, and such that $T$ is a member of the sequence. Then $T=T_{k}$, where $k=d^{\prime} T$.

By 4.14 and 4.43 the dimension is strictly increasing in the sequence

$$
\left(\theta T_{-1}, \theta T_{0}, \theta T_{1}, \ldots, \theta T_{d^{\prime}}\right)
$$

of flats of $M \times Z$. But $d \theta T_{-1}=-1$ and $d \theta T_{d^{\prime}}=d^{\prime}$, by 4.41. Hence $d \theta T_{i}=i$ for each member of the sequence. In particular $d \theta T=d^{\prime} T$.

We have shown that $\theta$ is a $1-1$ mapping of the set of flats of $M \cdot S$ onto a class of flats of $M \times Z$, and that $\theta$ preserves dimension and inclusion relations. We may say therefore that $\theta$ transforms each geometrical figure made up of flats of $M \cdot S$ into a geometrically equivalent figure made up of flats of $M \times Z$.

Not every flat of $M \times Z$ need be of the form $\theta T$ however. A simple example is provided by a matroid of three cells $a, b$, and $c$, and three atoms $\{a, b\}$, $\{b, c\}$, and $\{c, a\}$. We put $S=\{a, b\}$ and $Z=\{a, b, c\}$. Then $Z$ is clearly a carrier of $M \cdot S$. But the set $S$ is a flat of dimension 2 in $M \cdot S$ and dimension 1 in $M \times Z$. Hence $S$ cannot be of the form $\theta T$, with $T$ a flat of $M \cdot S$.

In general we have the following theorem

4.45 If $V$ is a flat of $M \times Z$, then $S \cap V$ is a flat of $M \cdot S$, and moreover $V \subseteq \theta(S \cap V)$.

PRoof: The first assertion follows from 1.11 and the definition of $M \cdot S$.

It is evident that $S \cap V \subset \theta(S \cap V)$.

Suppose $b \epsilon V-S$. There is an atom $X_{b}$ of $M \times Z$ such that $b \in X_{b}$ and $S \cap X_{b} \subseteq S \cap V$. Choose such an $X_{b}$ so that $\left|S \cap X_{b}\right|$ has the least possible value. Then $S \cap X_{b}$ is non-null, by 4.41 and 3.54 .

Assume $S \cap X_{b}$ is not an atom of $M \cdot S$. There exists $Y \epsilon M \cdot S$ such that $Y \subset S \cap X_{b}$. Then $b$ is not a cell of $\theta Y$, by the choice of $X_{b}$. Choose $a \in Y$. Applying Axiom II to $X_{b}$ and $\theta Y$ we find that $M \times Z$ has an atom $Y^{\prime}$ such that $b \in Y^{\prime} \subseteq\left(X_{b} \cup Y\right)-\{a\}$. But then $S \cap Y^{\prime}$ $\subset S \cap X_{b}$, and the definition of $X_{b}$ is contradicted.

We deduce that $S \cap X_{b}$ is an atom of $M \cdot S$. Hence $\theta\left(S \cap X_{b}\right)=X_{b}$, by 4.42, and therefore $b \epsilon \theta(S \cap V)$. Since this is true for all $b \epsilon V-S$ we have $V-S$ $\subset \theta(S \cap V)$.

Combining the above results we find that $V$ $\subset \theta(S \cap V)$.

\section{Specializations}

\subsection{Matroids and Chain-Groups}

As before we suppose given a commutative ring $R$ with a unit element and no divisors of zero.

We have to consider the following problem. Given a matroid $M$ we are to determine whether there exists a chain-group $N$ over $R$ such that $M=M(N)$. In this work we solve the problem only in some special cases, each time with the help of the following theorem. 5.11 Let $M$ be a matroid on a set $E$. With each atom $X$ of $M$ let there be associated a chain $f(X)$ on $E$ over $R$ such that $\|f(X)\|=X$. Suppose further that whenever $X, Y$, and $Z$ are distinct points on the same line of $M$ there exist non-zero elements $r$, $s$, and $t$ of $R$ such that

$$
r f(X)+s f(Y)+t f(Z)=0
$$


Let $N$ be the chain-group on $E$ over $R$ generated by the chains $f(X)$.

Then $M=M(N)$.

Proof: Let $T$ be any point of $M(N)$. There is an elementary chain $f$ of $N$ such that $\|f\|=T$. By the definition of $N$ we can find a flat $S$ of $M$ with the following properties.

(i) There is a nonzero element $r$ of $R$ such that $r f$ is a sum of chains $t f(X)$ with $X \subseteq S, t \in R$.

(ii) $d S$ has the least value consistent with (i).

If possible choose $a \epsilon S-T$ and write $S^{\prime}=\langle S-\{a\}\rangle$. The set $C$ of all points of $M$ which are subsets of $S^{\prime}$ is a linear subclass of $M$. (4.3).

If $g$ and $h$ are chains of $N$ we write

$$
g \equiv h \bmod S^{\prime}
$$

to denote that $g-h$ is a sum of chains $t f(X)$ such that $X \subseteq S^{\prime}$. In particular we may write

$$
\text { - } r f=\sum_{i} t_{i} f\left(Y_{i}\right) \bmod S^{\prime}, \quad t_{i} \in R \text {. }
$$

where the $Y_{i}$ are points of $M$ such that $a \epsilon Y_{i} \subseteq S$,

Let $Y$ be any point of $M$ such that $a \epsilon Y \subseteq S$. For each $Y_{i}$ the flat $Y \cup Y_{i}$ of $M$ is connected, by 3.48. Hence there is a path $P_{i}$ from $Y_{i}$ to $Y$ on $S$ such that no term of $P_{i}$ is a point of $C$, by 4.34 .

If $U$ and $V$ are consecutive points in $P_{i}$ then the line $U \cup V$ meets $S^{\prime}$ in a point $W$ of $C$, by 4.16 . Then by hypothesis we have

$$
x_{i} f(U) \equiv y_{i} f(V) \bmod S^{\prime},
$$

where $x_{i}$ and $y_{i}$ are nonzero members of $R$. If $Y_{i}$ $\neq Y$ we state this congruence for each pair of consecutive terms of $P_{i}$ and combine the results. In every case we have

$$
r_{i} f\left(Y_{i}\right) \equiv s_{i} f(Y) \bmod S^{\prime}
$$

where $r_{i}$ and $s_{i}$ are nonzero elements of $R$. Multiplying (1) by the product of the elements $r_{i}$ and using (2) we find that

$$
r^{\prime} f \equiv s^{\prime} f(Y) \quad \bmod S^{\prime}
$$

where $r^{\prime}$ and $s^{\prime}$ are elements of $R, r^{\prime}$ being nonzero.

Since $a$ does not belong to the domain of $f$ we deduce that $s^{\prime}=0$, which is contrary to the definition of $S$. We conclude that in fact $S=T$. Hence there is a point $T^{\prime}$ of $M$ on $S$ such that $T^{\prime} \subseteq T$.

Conversely let $T^{\prime}$ be any point of $M$. Then $f\left(T^{\prime}\right)$ is a chain of $N$. Hence there is a point $T$ of $M(N)$ such that $T \subseteq T^{\prime}$.

Applying Axiom I to these two results we find that the matroids $M$ and $M(N)$ are identical.

\subsection{Representative Matrices}

It is usually convenient to specify a chain-group over $R$ in the following way.
First we enumerate the set $E$, supposed non-null, as $\left\{e_{1}, e_{2}, \ldots, e_{n}\right\}$. For each chain $f$ on $E$ over $R$ we define the representative vector of $f$ as the l-rowed matrix.

$$
\left\{f\left(e_{1}\right), f\left(e_{2}\right), \ldots ., f\left(e_{n}\right)\right\} .
$$

Clearly linear relations between chains hold also for their representative vectors, and conversely.

A matrix $K$, with elements in $R$, is called a representative matrix of $N$ if it satisfies the following conditions.

(i) The rows of $K$ are linearly independent representative vectors of chains of $N$.

(ii) Every nonzero chain of $N$ has a representative vector which is a linear combination of rows of $K$.

Linear dependence is of course defined in terms of the elements of $R$ as coefficients.

A matrix $K$ satisfying (i) and (ii) completely determines the chain group $N$. It is also evident that any matrix $K$ with linearly independent rows can be interpreted as a representative matrix of a chain-group.

Condition (i) ensures that no row of $K$ consists solely of zero elements. If $N$ has only a zero chain it is convenient to say it has a null representative matrix, with no rows but $n$ columns. Such a matrix is considered to satisfy (i).

Let $K$ be a representative matrix of $N$ and let $S$ be any subset of $E$. We define $K(S)$ as the submatrix of $K$ made up of those columns which correspond to cells of $S$. If $S$ is null then $K(S)$ is a second kind of null matrix, being without columns but having as many rows as $K$. If the number of such rows is nonzero the matrix $K(S)$ is not regarded as having linearly independent rows.

As an immediate consequence of the definitions we have

5.21 Let $K$ be a representative matrix of $N$ and let $S$ be a subset of $E$ such that the rows of $K(S)$ are linearly independent. Then $K(S)$ is a representative matrix of $N \cdot S$.

Suppose the representative matrix $K$ of $N$ has $r$ rows and $n$ columns. It may happen that there is a subset $S$ of $E$, satisfying $|S|=r$ such that $K(S)$ is a unit matrix, that is having only l's in the main diagonal and only 0 's elsewhere. In this case $S$ is evidently a dendroid of $N$. We call $K$ a standard representative matrix of $N$ associated with the dendroid $S$. If we wish we may think of the unit matrix $K(S)$ as occupying the first $r$ columns of $K$. For this can be arranged by adjusting the initial enumeration of $E$.

5.22 Let $K$ be a standard representative matrix of $N$ associated with a dendroid $D$. Then the rows of $K$ are representative vectors of elementary chains of $N$, and these chains constitute a dendroid-basis of $N$ corresponding to $D$.

Proof: By 2.3 there is a dendroid basis $\left\{f_{a} \mid a \epsilon D\right\}$ of $N$. The theorem follows from the Corollary to 2.31 .

A null representative matrix of a trivial chain-group $N$ may be regarded as standard, being associated with a null dendroid.

Let $K$ be a standard representative matrix of $N$, 
associated with a dendroid $D$ and having $r$ rows and $n$ columns.

We construct a matrix $K^{*}$, having $n-r$ rows and $n$ columns, in the following way. The submatrix of $K^{*}$ occupying the $n-r$ columns corresponding in position to those of $K(E-D)$ in $K$ is a unit matrix. The other columns constitute a submatrix of $K^{*}$ equal to minus the transpose of $K(E-D)$. We illustrate the construction by an example, in which $R$ is the ring of integers

$$
\begin{aligned}
K & =\left\{\begin{array}{rrrrr}
1 & 0 & \overbrace{2-1} & 9 \\
0 & 1 & 0 & 5 & 6
\end{array}\right\} \\
K^{*} & =\left\{\begin{array}{rrrrr}
-2 & 0 & 1 & 0 & 0 \\
1 & -5 & 0 & 1 & 0 \\
-9 & -6 & 0 & 0 & 1
\end{array}\right\}
\end{aligned}
$$

$5.23 K^{*}$ is a standard representative matrix of $N^{*}$ associated with the dendroid $E-D$.

Proof: $E-D$ is a dendroid of $N^{*}$ by 2.43 . The theorem follows from 2.42 and 5.22.

Returning to the matrix $K$ we consider an arbitrary submatrix $A$ of $K(E-D)$. Let $A_{1}$ be the submatrix of $K$ constituted by those rows which meet $A$. Let $A_{2}$ be the submatrix of $A_{1}$ constituted by those of its columns which have $1^{\prime}$ s in $K(D)$. Thus $A_{2}$ is a unit matrix having the same number of rows as $A$. We refer to $A_{2}$ as the annex $\operatorname{Ann}(A)$ of $A$. The submatrix of $K$ made up of $A$ and Ann $(A)$ is the extension $\operatorname{Ext}(A)$ of $A$. We give an example below

D

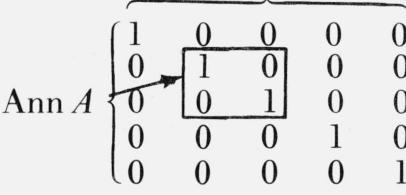

Figure 5A.

It is not of course essential that the rows and columns of $A$ should occur consecutively in $K$.

5.24 Let $A$ be any submatrix of $K(E-D)$. Then Ext $(A)$ is a standard representative matrix of a minor of $N$. The corresponding dendroid $D_{1}$ is made up of those cells which correspond to columns of Ann $(A)$.

Proof: Let $D_{1}$ and $Z$ be subsets of $E$ made up of the cells which correspond to columns of $\operatorname{Ann}(A)$ and $A$ respectively. Let $B$ be the submatrix of $K(E-D)$ made up of those rows of $K(E-D)$ which meet $A$. Thus Ann $(B)=$ Ann $(A)$.

We note that the rows of Ext $(B)$ and Ext $(A)$ are linearly independent. For each of these matrices contains the unit matrix $A n n(A)$ and has the same number of rows as Ann $(A)$.
It is clear that a chain $f$ of $N$ has a restriction in $N \times\left((E-D) \cup D_{1}\right)$ if and only if its representative vector is a linear combination of rows of $A_{1}$. Hence Ext $(B)$ is a representative matrix of $N \times\left((E-D) \cup D_{1}\right)$, and therefore $\operatorname{Ext}(A)$ is a representative matrix of the minor.

$$
N_{1}=\left(N \times\left((E-D) \cup D_{1}\right)\right) \cdot\left(Z \cup D_{1}\right),
$$

by 5.21. Evidently Ext $(A)$ satisfies the definition of a standard representative matrix of $N_{1}$ associated with $D_{1}$.

A minor of $N$ having a representative matrix of the form $\operatorname{Ext}(A)$, where $A$ is a submatrix of $K(E-D)$, will be said to be visible in $K$.

In general we cannot assert that there is a standard representative matrix of $N$ associated with each dendroid. However we have the following theorem.

5.25 Suppose that either $R$ is a field or that $N$ is regular. Then if $D$ is any dendroid of $N$ we can find a standard representative matrix of $N$ associated with $D$.

Proof: There is a dendroid-basis $\left\{f_{a} \mid a \in D\right\}$ of $N$. If $N$ is regular we can take each chain $f_{a}$ to be primitive. So in all cases covered by the theorem we may suppose $f_{a}(a)$ to have a reciprocal in $R$. Multiplying each chain $f_{a}$ by the reciprocal of $f_{a}(a)$ we obtain a dendroid-basis $\left\{g_{a} \mid a \in D\right\}$ of $N$ such that $g_{a}(a)=1$ for each $a \epsilon D$. Clearly there is a standard representative matrix of $N$, associated with $D$, whose rows are the representative vectors of the chains $g_{a}$.

We may now supplement 5.24 as follows.

5.26 Suppose that either $R$ is a field or $N$ is regular. Let $L$ be any minor of $N$. Then we can find a standard representative matrix $K$ of $N$, associated with a dendroid $D$, such that $L$ is visible in $K$.

Proof: Let $D_{1}$ be any dendroid of $L$ and let $Z$ be the set of all cells of $L$ not in $D_{1}$. Then we may write

$$
L=(N \times U) \cdot\left(Z \cup D_{1}\right),
$$

by 3.243 .

We recall that the dendroids of a chain-group $N$ are the dendroids of the corresponding matroid $M(N)$, by 2.3 .

Let $V$ be a carrier of $M(L)$, that is $(M(N) \times U)$ $\cdot\left(Z \cup D_{1}\right)$ by 3.31 , in $M(N) \times U$. Then $D_{1}$, being a dendroid of $M(L)$, is also a dendroid of $M(N) \times V$. Hence $D_{1}$ is a dendroid of $N \times V$.

We have

$$
L=(N \times V) \cdot\left(Z \cup D_{1}\right),
$$

for the restrictions to $Z \cup D_{1}$ of the members of a dendroid basis of $N \times V$ corresponding to $D_{1}$ evidently form a dendroid-basis of $L$. (2.31, Corollary).

Let $D_{2}$ be a dendroid of $N \cdot(E-V)$, and write $D_{1} \cup D_{2}=D$. Then $D$ is a dendroid of $N$, by 3.31 and 3.53. Let $K$ be a standard representative matrix of $N$ associated with $D$. Such a matrix exists, by 5.25.

Let $f$ be any chain of $N \times\left(E-D_{2}\right)$. Its restriction to $E-V$ has a domain not meeting $D_{2}$, and is therefore zero. Since $E-D_{2}=(E-D) \cup D_{1}$ we deduce that 


$$
L=\left(N \times\left((E-D) \cup D_{1}\right)\right) \cdot\left(Z \cup D_{1}\right)
$$

But by the argument of 5.24 this minor is visible in $K$.

\subsection{Characterization of Binary Matroids}

A binary matroid is by definition the matroid of a binary chain-group $N$, that is a chain-group over the field $G F(2)$ of residues mod 2.

5.31 Let $M$ be any binary matroid. Then $M^{*}$ and the minors of $M$ are binary. (2.66 and 3.31).

A binary matroid is conveniently specified by giving a representative matrix of the associated binary chaingroup.

5.32 Let $N$ be a binary chain-group on a set $E$. Then every nonzero chain of $N$ is a sum of elementary chains of $N$ with disjoint domains.

Proof: If possible let $f$ be a nonzero chain of $N$ for which the theorem fails, and for which $\|f\|$ has the least number of cells consistent with this condition.

There is an elementary chain $g$ of $N$ such that $\|g\| \subseteq\|f\|$. But $g \neq f$, for otherwise $f$ would satisfy the theorem. Hence the chains $g$ and $f+g$ have disjoint domains, each with fewer cells than $\|f\|$. Accordingly $g$ and $f+g$ satisfy the theorem, and therefore $f$ satisfies it, contrary to assumption. The theorem follows.

In a binary chain-group there is little distinction between a chain and its domain. If $S_{1}, S_{2}, \ldots, S_{k}$ are subsets of $E$, not necessarily all distinct, we define their $\bmod 2$ sum as the subset $S$ of $E$ such that $a \epsilon S$ if and only if the number of suffices $i, 1 \leqslant i \leqslant k$, satis. fying $a \epsilon S_{i}$ is odd. This addition of subsets is evidently commutative and associative, and we use the ordinary additive notation for it. Chains $f_{i}$ on $E$ over $G F(2)$ evidently satisfy

5.33

$$
\left\|\sum_{i=1}^{k} f_{i}\right\|=\sum_{i=1}^{k}\left\|f_{i}\right\| \cdot
$$

We conclude this section with two theorems characterizing binary matroids.

5.34 A matroid $M$ on $E$ is binary if and only if any non-null mod 2 sum of atoms of $M$ is a union of disjoint atoms of $M$.

Proof: Suppose $M=M(N)$, where $N$ is a binary chain-group. A non-null $\bmod 2$ sum of atoms of $M$ is the domain of a non-null chain of $N$, by 5.33. This is a union of disjoint atoms of $M$ by 5.32 .

Conversely suppose $M$ satisfies the stated condition. Let $N$ be the chain-group generated by those chains on $E$ over $G F(2)$ whose domains are atoms of $M$. Clearly each atom of $M$ contains an atom of $M(N)$. On the other hand each atom of $M(N)$ is a mod 2 sum of atoms of $M$ and therefore contains an atom of $M$. Hence $M=M(N)$, by Axiom I, and so $M$ is binary.

$5.35 A$ matroid $M$ is binary if and only if each connected line of $M$ is on exactly three points.

Proof: Suppose $M$ is binary. Let $L$ be any connected line of $M$. There are at least three distinct points on $L$, by 4.23 . Denote two of them by $X$ and $Y$, and let $Z$ be any other.

Since $X \cup Z=L=X \cup Y$, by 4.22 , we have $Y-X \subseteq Z$. Similarly $X-Y \subseteq Z$. Hence $Z$ contains the $\bmod 2$ sum $X+Y$, which is non-null by Axiom I. But $X+Y$ is a union of disjoint atoms of $M$, by 5.34. Hence $Z=X+Y$, by Axiom $\mathrm{I}$. Thus $L$ is on just one point other than $X$ and $Y$.

Conversely suppose there are just three points on each connected line of $M$. If $X$ is any point (atom) of $M$ we write $f(X)$ for the chain on $E$ over $G F(2)$ whose domain is $X$. Let $N$ be the binary chain-group on $E$ generated by the chains $f(X)$.

Let $X, Y$, and $Z$ be the three points on any connected line $L$ of $M$. Then $X+Y+Z$ is null, by 4.21 . Hence $f(X)+f(Y)+f(Z)=0$, by 5.33. Accordingly $M=M(N)$, by 5.11 , and so $M$ is binary.

\subsection{Regular Chain-Groups and Matroids}

A regular matroid is by definition the matroid of a regular chain-group $N$, as defined in section 1.2. 5.41 Let $M$ be any regular matroid. Then $M^{*}$ and the minors of $M$ are regular. (2.47, 2.66, 3.26 and 3.31).

Let $f$ and $g$ be two integral chains on a set $E$. We say that $f$ conforms to $g$ if $\|f\| \subseteq\|g\|$ and $f(a) g(a)>0$ for each $a \epsilon|| f||$.

5.42 Let $N$ be a regular chain-group on $E$, and let $f$ be a nonzero chain of $N$. Then there exists a primitive chain of $N$ conforming to $f$.

Proof: If possible choose $f$ so that the theorem fails and $\|f\|$ has the least number of elements consistent with this condition.

Since $N$ is regular it has a primitive chain $g$ such that $\|g\| \subseteq\|f\|$. Choose $a \epsilon\|g\|$ such that $f(a)$ has the least possible absolute value. Replacing $g$ by $-g$ if necessary we arrange that $g(a) \cdot f(a)>0$. Write

$$
h=f-f(a) g(a) g
$$

If $h=0$ then $f$ is a positive multiple of $g$, and so $g$ conforms to $f$. If $h \neq 0$ it is clear that $h$ conforms to $f$. Moreover $\|h\|$ then has fewer cells that $\|f\|$ and so there is a primitive chain $k$ of $N$ conforming to $h$. But then $k$ conforms also to $f$. So in each case the choice of $f$ is contradicted. The theorem follows:

5.43 Let $N$ be a regular chain-group on $E$, and let $f$ be a nonzero chain of $N$. Then $f$ can be represented as a sum of primitive chains of $N$, each conforming to $f$.

Proof: Let $Z(k)$, where $k$ is a chain of $N$, denote the sum of the absolute values of the coefficients in $k$.

If possible choose $f$ so that the theorem fails and $Z(f)$ has the least value consistent with this. By 5.42 there is a primitive chain $g$ of $N$ conforming to $f$. Write $h=f-g$. If $h=0$ then $f=g$. If $h \neq 0$ it is clear that $Z(h)<Z(f)$ and that $h$ conforms to $f$. Hence $h$ is a sum of primitive chains of $N$, each conforming to $h$ and therefore to $f$. In either case the definition of $f$ is contradicted.

Let $f$ and $g$ be integral chains on $E$ and let $q$ be an integer not less than 2 . We call $g$ a $q$-representative of $f$ if the following conditions are satisfied for each $a \epsilon E$.

(i) $g(a) \equiv f(a) \bmod q$,

(ii) $|g(a)|<q$. 
5.44 Let $f$ be a chain of a regular chain-group $N$ on $E$. Then for each integer $\mathrm{q} \geqslant 2$ some chain of $N$ is a $q$-representative of $f$.

Proof: There is at least one chain $g$ of $N$ which satisfies condition (i), namely $f$. For each such $g$ we define $Y(g)$ as the number of cells $a \epsilon E$ satisfying $|g(a)| \geqslant q$. We choose a particular $g$ satisfying (i) so that $Y(g)$ has the least possible value.

If $Y(g)>0$ choose $b \in E$ so that $|g(b)| \geqslant q$. By 5.43 there is a primitive chain $h$ of $N$ conforming to $g$ and satisfying $h(b)= \pm 1$. Write

$$
g_{1}=g-q h \text {. }
$$

Clearly $g_{1}$ satisfies (i). Moreover we have

$$
\begin{gathered}
\left|g_{1}(b)\right|<|g(b)|, \\
(|g(a)|<q) \rightarrow\left(\left|g_{1}(a)\right|<q\right) .
\end{gathered}
$$

Hence $Y\left(g_{1}\right) \leqslant Y(g)$, with equality only if $\left|g_{1}(b)\right| \geqslant q$.

If $\left|g_{1}(b)\right| \geqslant q$ we repeat the process with $g_{1}$ replacing $g$, and with the same choice of $b$. Proceeding in this way we eventually obtain a chain $g^{\prime}$ of $N$ such that $g^{\prime}$ satisfies (i) and $Y\left(g^{\prime}\right)<Y(g)$. But this is contrary to the choice of $g$. We deduce that in fact $Y(g)=0$. The theorem is thus true.

We use 5.44 to prove the following important theorem about regular matroids.

5.45 Every regular matroid is binary.

Proof: Let $M$ be a regular matroid. Then $M=M(N)$, where $N$ is a regular chain-group. For each chain $f$ of $N$ let $f^{\prime}$ be the chain over $G F(2)$ derived from it by replacing each coefficient in $f$ by its residue $\bmod 2$. Then the chains $f^{\prime}$ constitute a binary chaingroup $Q$.

Let $X$ be any atom of $M$. It is the domain of a primitive chain of $N$, and therefore of a nonzero chain of $Q$. There is an atom $Y$ of $M(Q)$ such that $Y \subseteq X$.

Conversely let $Y$ be an atom of $M(Q)$. There is a chain $f$ of $N$ such that $Y$ is the domain of $f^{\prime}$. But there is a chain $g$ of $N$ which is a 2-representative of $f$, by 5.44 , and we have $\|g\|=\left\|f^{\prime}\right\|=Y$. Hence there is an atom $X$ of $M=M(N)$ such that $X \subseteq Y$.

Applying Axiom I we find that $M=\bar{M}(Q)$. Thus $M$ is binary.

There are some important properties of regular chain-groups which are expressible in terms of their standard representative matrices.

5.46 Let $N$ be a regular chain-group on $E$. Let $D$ be any dendroid of $N$. Let $K$ be a standard representative matrix of $N$ associated with $D$, and let $S$ be any subset of $E$ such that $|S|=|D|$. Then the determinant of the square submatrix $K(S)$ is \pm 1 if $S$ is a dendroid of $N$, and zero otherwise.

Proof: It is clear that $S$ meets the domain of every nonzero chain of $N$ if and only if $K(S)$ is nonsingular. So, by $2.23 \operatorname{det} K(S)$ is nonzero if and only if $S$ is a dendroid of $N$

Suppose $K(S)$ is nonsingular. Let $K_{1}$ be the standard representative matrix of $N$ associated with $S$ (5.25). Since the rows of $K_{1}$ are linear combinations of those of $K$ there is a square matrix $A$ of integers such that $\mathrm{A} K=K_{1}$. But then $A K(S)=K_{1}(S)$. On taking determinants we have

$$
\operatorname{det} A \cdot \operatorname{det} K(S)=1,
$$

since $K_{1}(S)$ is a unit matrix. Since $A$ and $K(S)$ are matrices of integers it follows that $\operatorname{det} K(S)= \pm 1$.

Using the formula for the determinant of a product of two rectangular matrices we can deduce from 5.46 that the number of distinct dendroids of $N$ is

$$
\operatorname{det}\left(K K^{T}\right) \text {, }
$$

where $K^{T}$ is the transpose of $K$. This generalizes a well-known formula for the number of spanning trees in a graph $G$.

There is a converse of 5.46 to the effect that if $K$ is an $r$-rowed matrix of integers whose rows are linearly independent, and in which the determinants of the $r \times r$ submatrices are restricted to the values $1,-1$, and 0 , then $K$ is a representative matrix of a regular chain group. We do not use this theorem in what follows, and we leave its proof as an exercise to the reader.

The following consequence of 5.46 is of interest. 5.47 Let $N$ be a regular chain-group on $E$. Let $K$ be a standard representative matrix of $N$ associated with a dendroid $D$. Then the determinants of the square submatrices of $K(E-D)$ are restricted to the values 1 , -1 and 0.

Proof: Let $A$ be such a square submatrix. Let $T$ be the subset of $E$ corresponding to the columns of $K(E-D)$ meeting $A$ and those of $K(D)$ not meeting Ann $(A)$.

Now $K(T)$ is square, and det $K(T)=1,-1$ or 0 by 5.46. But expansion of det $K(T)$, using the columns in $K(D)$, shows that det $K(T)= \pm \operatorname{det} A$. The theorem follows.

We can express this result by saying that $K(E-D)$ is completely unimodular. Evidently $K$ is itself completely unimodular.

\subsection{Some Binary Matroids Which Are Not Regular}

In this section we show that the converse of 5.45 is not true. We do so by using the following observation. 5.51 Let $K$ be a standard representative matrix, associated with a dendroid $D$, of a binary chain-group $N$ on $E$. Then if $M(N)$ is regular we can replace each zero element of $K$ by the integer 0 , and each nonzero element by an integer +1 or -1 in such a way as to transform $K(E-D)$ into a completely unimodular matrix.

Proof: If $M$ is regular we can write $M=M(N)$ $=M\left(N_{1}\right)$, where $N_{1}$ is a regular chain-group.

Each row of $K$ is the representative vector of an elementary chain of $N$. We replace it by the representative vector of a primitive chain of $N_{1}$ with the same domain. Performing this operation for each row, and then making appropriate multiplications of rows by -1 we evidently transform $K$ into a standard representative matroid $K_{1}$ of $N_{1}$ associated with $D$. 
We observe that $K$ is transformed into $K_{1}$ by an operation of the kind specified, and that $K(E-D)$ is transformed into the completely unimodular matrix $K_{1}(E-D)$, by 5.47 .

We now investigate a binary chain-group $N$ with the standard representative matrix shown below

$$
\left\{\begin{array}{lllllll}
1 & 0 & 0 & 1 & 0 & 1 & 1 \\
0 & 1 & 0 & 1 & 1 & 0 & 1 \\
0 & 0 & 1 & 0 & 1 & 1 & 1
\end{array}\right\}
$$

We shall describe a binary matroid corresponding to such a chain-group as being "of type BI".

5.52 No matroid of Type BI is regular.

Proof: Suppose the theorem false. Then by 5.51 there is a completely unimodular matrix $A$ of the following form.

$$
\left\{\begin{array}{llll}
x_{11} & 0 & x_{13} & x_{14} \\
x_{21} & x_{22} & 0 & x_{24} \\
0 & x_{32} & x_{33} & x_{34}
\end{array}\right\}
$$

Here each $x_{i j}$ is +1 or -1 .

The determinant of the square submatrix

$$
\left\{\begin{array}{ll}
x_{11} & x_{14} \\
x_{21} & x_{24}
\end{array}\right\}
$$

is clearly even. It is therefore zero since $A$ is completely unimodular. We deduce that $x_{11} x_{24}=x_{14} x_{21}$, that is

$$
x_{11} x_{21} x_{14} x_{24}=1 \text {. }
$$

Similarly we have

$$
\begin{aligned}
& x_{22} x_{32} x_{24} x_{34}=1, \\
& x_{13} x_{33} x_{14} x_{34}=1 .
\end{aligned}
$$

We have also

$$
\left|\begin{array}{lll}
x_{11} & 0 & x_{13} \\
x_{21} & x_{22} & 0 \\
0 & x_{32} & x_{33}
\end{array}\right|=x_{11} x_{22} x_{33}+x_{13} x_{21} x_{32}
$$

But this number is even, and therefore zero by the complete unimodularity of $A$. Hence

$$
x_{11} x_{21} x_{22} x_{32} x_{13} x_{33}=-1
$$

But equations (1), (2), (3), and (4) are inconsistent, for they imply

$$
\begin{aligned}
-1 & =\left(x_{11} x_{21} x_{22} x_{32} x_{13} x_{33}\right) x_{14}^{2} x_{24}^{2} x_{34}^{2} \\
& =\left(x_{11} x_{21} x_{14} x_{24}\right)\left(x_{22} x_{32} x_{24} x_{34}\right)\left(x_{13} x_{33} x_{14} x_{34}\right) \\
& =1 .
\end{aligned}
$$

The theorem follows.

The dual of a matroid of Type BI will be said to be "of Type BII". Such a matroid is binary but not regular, by $5.31,5.41$, and 2.65 .

By another application of 5.31 and 5.41 we have 5.53 Let $M$ be a binary matroid having a minor of Type BI or BII. Then $M$ is not regular.

Our next main task is the proof of the converse of this theorem, that if a binary matroid is not regular it must have a minor of Type BI or BII. Our proof will be based on 5.11. But the question whether it is possible to assign suitable integral chains $f(X)$ to the atoms $X$ of a given binary matroid raises grave difficulties. Apparently these can only be resolved by the use of the homotopy theory developed in the next Chapter.

\subsection{Graphic and Cographic Matroids}

We have discussed specializations from general matroids to binary matroids, and from binary matroids to regular ones. The next step in this progression brings us to the graphic and cographic matroids.

We have defined a graphic matroid as one which can be interpreted as the bond-matroid of a graph (sec. 2.5). A cographic matroid is one which can be interpreted as the polygon-matroid of a graph.

5.61 The cographic matroids are the duals of the graphic matroids. (2.67).

5.62 Let $M$ be any graphic (cographic) matroid. Then the minors of M are graphic (cographic). (3.321, 3.322, 3.371, 3.372).

5.63 Every graphic or cographic matroid is regular. $(1.24,5.41$ and 5.61).

The last of these theorems shows that the graphic and cographic matroids can be regarded as specializations of the regular ones. We shall therefore have to discuss the question: when is a regular matroid graphic? Here we shall only state the result, leaving the proof for a later chapter.

A complete 5-graph, or 5-clique is a graph having just five vertices $a_{1}, \ldots ., a_{5}$ and just ten edges $L_{i j}$, the ends of $L_{i j}$ being $a_{i}$ and $a_{j}(1 \leqslant i<j \leqslant 5)$. A Thomsen graph, or 4-cage is a graph having just six vertices $a_{1}, a_{2}, a_{3}, b_{1}, b_{2}, b_{3}$ and just nine edges $L_{i j}$, the ends of $L_{i j}$ being $a_{i}$ and $b_{j} . \quad(1 \leqslant(i, j) \leqslant 3)$. These two graphs are illustrated in figure $5 \mathrm{~B}$.

These two graphs are often spoken of as the Kuratowski graphs, in view of Kuratowski's Theorem that every nonplanar graph contains a subdivision of one of them.

We shall prove in a later chapter that a regular matroid $M$ is nongraphic if and only if one of its minors 

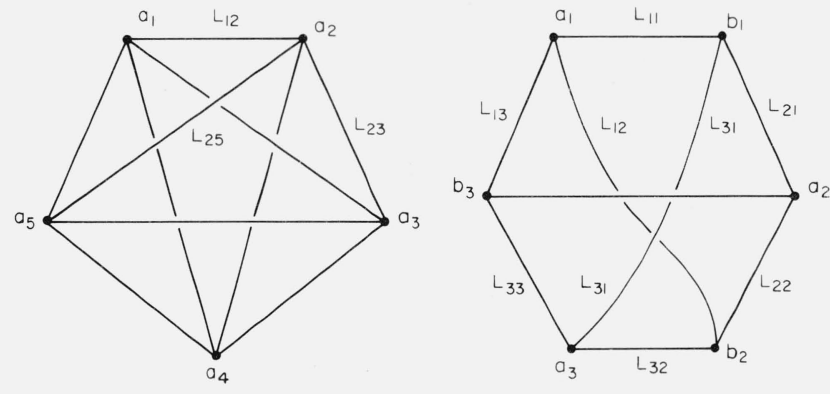

Figure 5B

is the polygon-matroid of a Kuratowski graph. Dually $M$ is noncographic if and only if one of its minors is the bond-matroid of a Kuratowski graph.

\section{Homotopy}

\subsection{Elementary Re-entrant Paths}

Throughout this chapter we suppose given a matroid $M$ and a linear subclass $C$ of $M$. We describe a path in $M$ as being off $C$ if it has no term which is a vertex of $C$, and we study the properties of those re-entrant paths in $M$ which are off $C$. As a special case of course we can put $C=\phi$ and study the set of all reentrant paths of $M$.

We specify four kinds of re-entrant path off $C$ as fundamental, or elementary, and we show that every re-entrant path off $C$ can, in a sense to be explained, be expressed as a combination of elementary ones.

The first kind of elementary re-entrant path consists of all paths off $C$ of the form $(X, Y, X)$. The second consists of all paths off $C$ of the form $(X, Y, Z, X$ where $d(X \cup Y \cup Z) \leqslant 2$.

Suppose $P$ is a plane of $M$ on which there are two distinct points $A$ and $B$ of $C$ such that each connnected line on $P$ is on either $A$ or $B$. Then any path, on $P$ and off $C$, of the form $(X, Y, Z, T, X)$ where $X, Y, Z$, and $T$ are distinct, $X \cup Y$ and $Z \cup T$ are lines on $A$, and $Y \cup Z$ and $T \cup X$ are lines on $B$, is an elementary reentrant path of the third kind.

Such a path is shown in figure 6A. In this and other diagrams we adopt the convention that points of $C$ are to be represented by 4-pointed stars.

Now let $J$ be a 3-flat of $M$ on which there are three points $Z_{1}, Z_{2}$, and $Z_{3}$ such that $Z_{1} \cup Z_{2}, Z_{2} \cup Z_{3}$ and $Z_{3} \cup Z_{1}$ are disconnected lines. Let there be just six connected planes on $J$, two on each of these lines. We enumerate the planes as $P_{1}, P_{2}, \ldots, P_{6}$ in such a way that

$$
\left\langle P_{i} \cap P_{i+3}\right\rangle=Z_{j} \cup Z_{k},
$$

where $(i, j, k)$ is any permutation of $(1,2,3)$.

If $1 \leqslant i<j \leqslant 6$ the flat $\left\langle P_{i} \cap P_{j}\right\rangle$ is a line, by 4.172 .

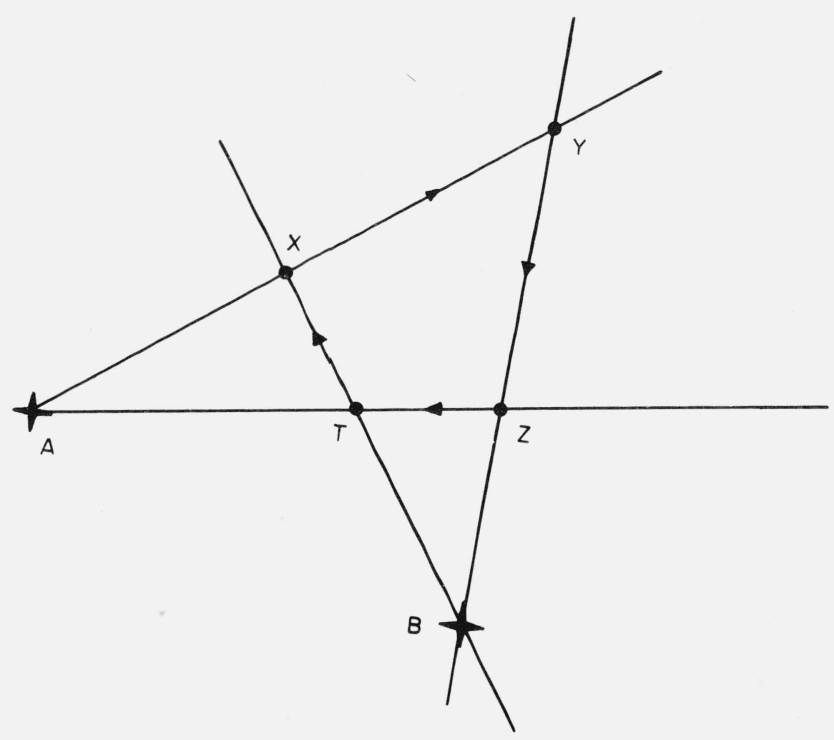

Figure 6A

We denote it by $L_{i j}$. If $j=i+3$ then $L_{i j}$ is the disconnected line

$$
\left(Z_{1} \cup Z_{2} \cup Z_{3}\right)-Z_{i} .
$$

If $j \neq i+3$ let $k$ be that integer 1,2 , or 3 which is not congruent to $i$ or $j \bmod 3$. Then $L_{i j}$ is on $Z_{k}$, and it meets $P_{k}$ and $P_{k+3}$ in two distinct points. Hence $L_{i j}$ is connected by 4.23 . Clearly it is on no connected plane on $J$ other than $\boldsymbol{P}_{i}$ and $\boldsymbol{P}_{j}$.

We observe that $J=P_{1} \cup P_{2}$, by 4.14 , and is thus connected, by 3.48. It follows that each connected line on $J$ is on two connected planes of $J$. Hence the 12 lines $L_{i j}, j \neq i+3$, are the only connected lines on $J$.

We write

$$
X_{i j k}=\left\langle P_{i} \cap P_{j} \cap P_{k}\right\rangle
$$

for $1 \leqslant i<j<k \leqslant 6$. Then $X_{i j k}$ is a point on $J$, by 4.173 , being identical with $\left\langle L_{i j} \cap P_{k}\right\rangle$. If two of the suffices $i, j$, and $k$ are congruent $\bmod 3$, then $X_{i j k}$ is one of the points $Z_{1}, Z_{2}$, and $Z_{3}$. The remaining eight points $X_{i j k}$ are all distinct, for on any one of them there can be only three planes such that each is on $L_{14}, L_{25}$, or $L_{36}$. These eight points, together with $Z_{1}, Z_{2}$, and $Z_{3}$ are the only points on $J$. For each point on $J$ is on three distinct connected planes of $J$, by two applications of 4.26 .

The geometrical figure just described is shown in figure $6 \mathrm{~B}$. Three lines are broken to indicate disconnection.

We-refer to this structure as a box, saying that the planes $P_{i}$ are its sides, the 12 connected lines are its edges, the points $Z_{i}$ are its radiants and the other eight points are its corners. 


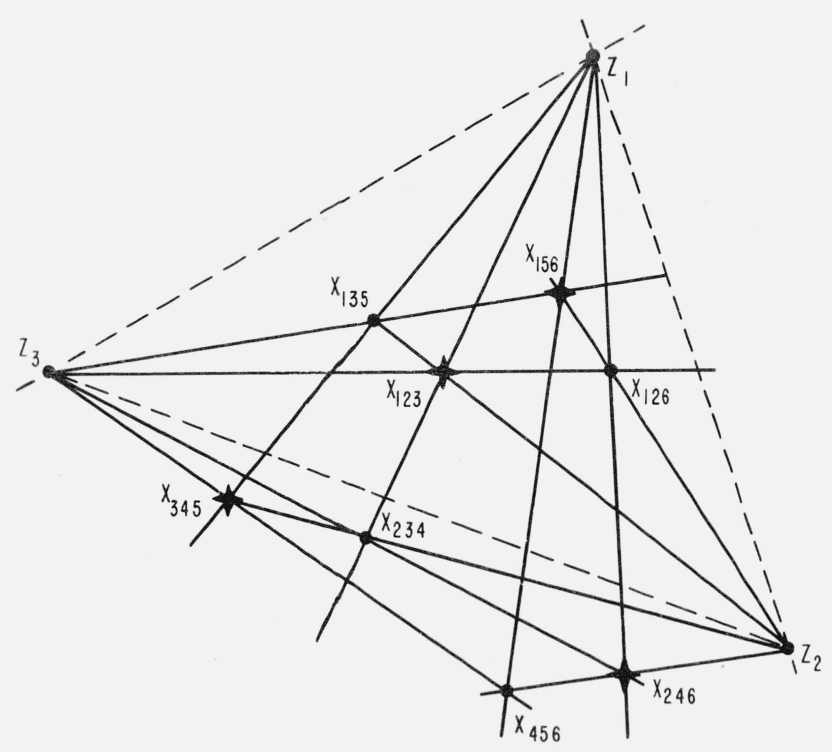

Figure 6B

It may happen that the points of $C$ on $J$ are four corners of the box. These corners must then occur alternately and we may suppose them to be $X_{123}, X_{246}$, $X_{345}$, and $X_{156}$, as shown in the diagram. These are the four points $X_{i j k}$ such that no two suffices are congruent $\bmod 3$ and the number of suffices less than 4 is odd.

Under these circumstances any path of the form ( $A, X, B, Y, A)$, where $A$ and $B$ are radiants of the box, and $X$ and $Y$ are corners not in $C$, is called an elementary re-entrant path of the fourth kind.

\subsection{Homotopy}

Suppose we have two paths $P R$ and $P Q R$ off $C$, where $Q$ is an elementary re-entrant path of the $k$ th kind. Then we call the process of deriving one of these paths from the other an elementary deformation of the $k$ th kind. We say that two given paths $P$ and $Q$ off $C$ are homotopic if one can be derived from the other by a finite sequence of elementary deformations. We write this relation as $P \sim Q$, or if it is necessary to specify $C$ as $P \sim Q \bmod C$. If a path $P$, necessarily re-entrant, is homotopic to a degenerate path we say that $P$ is null-homotopic. We write this relation as $P \sim 0$, or as $P \sim 0 \bmod C$.

Homotopy is clearly an equivalence relation.

We note some simple rules of computation.

6.21 If two path-products $P Q R$ and $P Q_{1} R$ off $C$ are such that $Q \sim Q_{1}$, then $P Q R \sim P Q_{1} R$.

Proof: Any sequence of elementary deformations transforming $Q$ into $Q_{1}$ must also transform $P Q R$ into $P Q_{1} R$.

6.22 If $P$ is any path off $C$ then $P P^{-1} \sim 0$.

Proof: If possible choose $P$ so that the theorem fails and $s(P)$ has the least value consistent with this condition.
If $s(P)=0$ the theorem is trivially true. If $s(P)=1$ it is true because $P P^{-1}$ is an elementary re-entrant path of the first kind. We deduce that $s(P) \geqslant 2$.

We can now write $P=Q R$, where $s(Q)$ and $s(R)$ are both less than $s(P)$. We have $Q Q^{-1} \sim 0$ and $R R^{-1} \sim 0$, by the choice of $P$. But now

$$
P P^{-1} \sim Q R R^{-1} Q^{-1} \sim Q Q^{-1} \sim 0,
$$

by 6.21. The theorem follows.

6.23 If PUR and PVR are paths off $C$ such that $U V^{-1}$ $\sim 0$, then $P U R \sim P V R$.

Proof: $P U R \sim P U V^{-1}\left(V^{-1}\right)^{-1} R$, by 6.21 and 6.22 ,

$\sim P U V^{-1} V R$, by 4.321 ,

$\sim P V R$, by 6.21 .

In what follows we prove that every re-entrant path on $M$ and off $C$ is null-homotopic,

It will be convenient to denote by $H(n)$ the proposition that every re-entrant path off $C$ which is on a flat of $M$ of dimension $\leqslant n$ is null-homotopic.

Any path $P=\left(X_{1}, X_{2}, \ldots, X_{k}\right)$ of $M$ determines a flat $F(P)$ satisfying

$$
F(P)=X_{1} \cup X_{2} \cup \ldots \cup X_{k} .
$$

6.24 If $P$ is any path of $M$ then the flat $F(P)$ is connected.

Proof: $X_{i} \cup X_{i+1}$ is a connected line whenever $1 \leqslant i<k$. Hence the theorem follows by repeated application of 3.48 .

We refer to the dimension of $F(P)$ also as the $d i$ mension of $P$.

\subsection{A Lemma}

We devote this section to a proof of the following proposition.

6.31 Suppose $n \geqslant 2$ and $H(n)$ is true. Let $Q=(W, X$, $Y, Z, W)$ be a path off $C$ of dimension $n+1$ such that $W \cup X \cup Y$ and $Y \cup Z \cup W$ are connected planes and $W \cup Y$ is a disconnected line. Then $Q \sim 0$.

We write $F_{1}=W \cup X \cup Y$ and $F_{2}=Y \cup Z \cup W$.

We arrive at a proof of 6.31 by way of a chain of minor propositions.

6.311 Let $Q^{\prime}=\left(W, X^{\prime}, Y, Z^{\prime}, W\right)$ be a path off $C$ such $X^{\prime}$ is on $F_{1}$ and $Z^{\prime}$ on $F_{2}$. Then $Q^{\prime} \sim Q$.

Proof: This situation is illustrated in figure $6 \mathrm{C}$. The proof runs as follows:

$Q^{\prime} \sim\left(W, X^{\prime}, Y\right)(Y, X, W)(W, X, Y)(Y, Z, W)(W, Z, Y)$

$\left(Y, Z^{\prime}, W\right)$,

by 6.21 and 6.22 ,

$=\left(W, X^{\prime}, Y, X, W\right)(W, X, Y, Z, W)\left(W, Z, Y, Z^{\prime}, W\right)$

$$
\sim(W, X, Y, Z, W)=Q,
$$

by $H(n)$, since $\left(W, X^{\prime}, Y, X, W\right)$ and $\left(W, Z, Y, Z^{\prime}, W\right)$ are on planes. 


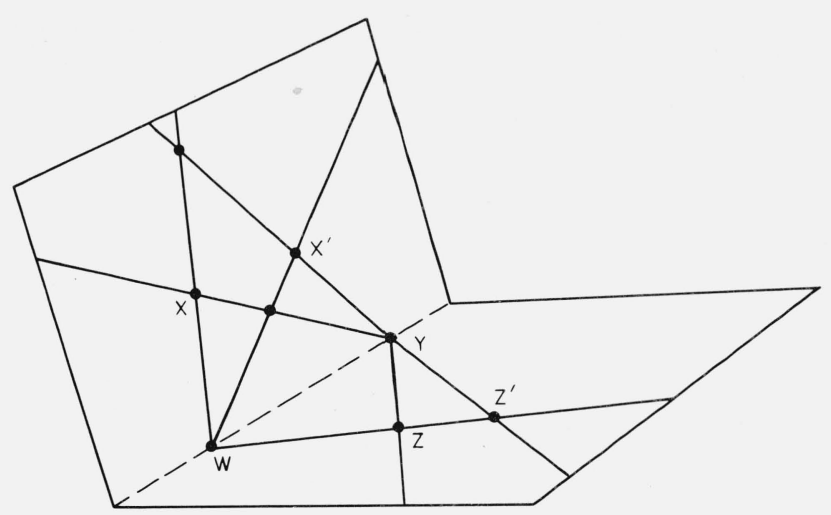

Figure 6C

A transversal of dimension $n$ is a connected $n$-flat of $M$ which is on $F(Q)$ but not on both $W$ and $Y$. Such a transversal meets each of $F_{1}$ and $F_{2}$ in a connected line, by $4.14,4.16$, and 4.281 .

A transversal of dimension $n-1$ is a connected $(n-1)$-flat of $M$ which is on $F(Q)$ but not on $W$ or $Y$. By 4.14 and 4.16 the transversal has just one common point with each of $F_{1}$ and $F_{2}$. We call these two points the poles of the transversal.

Let $B$ be a transversal of dimension $n-1$ with poles $X^{\prime}$ on $F_{1}$ and $Z^{\prime}$ on $F_{2}$. Using 4.26 we find that $B$ is on two distinct connected $n$-flats on $F(Q)$. By 4.14 and 4.16 these are $B \cup W$ and $B \cup Y$, and each of them is a transversal of dimension $n$. Their connected lines of intersection with $F_{1}$ and $F_{2}$ are $X^{\prime} \cup W, X^{\prime} \cup Y$, $Z^{\prime} \cup W$ and $Z^{\prime} \cup Y$.

6.312 Let $B$ be a transversal of dimension $n$. Then either $Q \sim 0$ or $B$ has a pole in $C$.

Proof: Let the poles of $B$ be $X^{\prime}$ on $F_{1}$ and $Z^{\prime}$ on $F_{2}$. Suppose neither of them is in $C$.

By 4.34 there is a path $R$ from $X^{\prime}$ to $Z^{\prime}$ on $B$ which is off $C$. Hence there are paths $\left(W, X^{\prime}\right) R\left(Z^{\prime}, W\right)$ and $\left(X^{\prime}, Y, Z^{\prime}\right) R^{-1}$ on the transversals $B \cup W$ and $B \cup Y$ respectively of dimension $n$. We now have

$$
\begin{aligned}
Q & \sim\left(W, X^{\prime}, Y, X^{\prime}, W\right), \text { by } 6.311 \\
& =\left(W, X^{\prime}\right)\left(X^{\prime}, Y, Z^{\prime}\right)\left(Z^{\prime}, W\right) \\
& \sim\left(W, Z^{\prime}\right) R^{-1} R\left(Z^{\prime}, W\right), \text { by } 6.23 \text { and } H(n), \\
& \sim\left(W, Z^{\prime}, W\right) \text { by } 6.22 \\
& \sim 0,
\end{aligned}
$$

by an elementary deformation of the first kind. This proves the proposition.

Let us make the assumption that $Q$ is not nullhomotopic.

By 4.27 there is a transversal $A$ of dimension $n$ which is not on $Y$. Let its lines of intersection with $F_{1}$ and $F_{2}$ be $L_{1}$ and $L_{2}$ respectively. By the definition of a linear subclass there is a point $X^{\prime}$, other than $W$, on $L_{1}$ and not in $C$. By 4.27 there is a connected $\left(n-1\right.$ ) flat $B$ on $M$ which is on $A$ and $X^{\prime}$ but not on $W$.

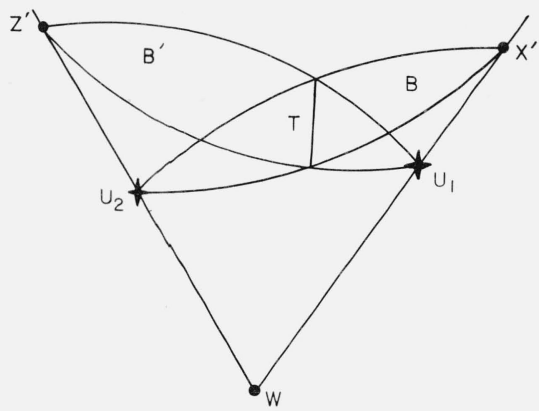

Figure 6D

Now $B$ is a transversal of dimension $n-1$. Let its pole on $L_{2}$ be $U_{2}$. Then $U_{2} \epsilon C$, by 6.312 .

Similarly there is a transversal $B^{\prime}$ of dimension $n-1$ on $A$ having a point $Z^{\prime}$ not in $C$ as its pole on $L_{2}$ and a point $U_{1}$ of $C$ as its pole on $L_{1}$. We write $T=\left\langle B \cap B^{\prime}\right\rangle$. By 4.14 and $4.16 T$ is an $(n-2)$-flat on A. (See fig. 6D.)

Let $\Sigma$ be the class of all points on $T$ which are not in $C$.

$6.313 \Sigma$ is non-null.

Proof: Since $T$ is an $(n-2)$ flat on an $(n-1)$-flat $B$, and since $B$ has a point $U_{2}$ in $C$ and a point $X^{\prime}$ not in $C$, this follows from 4.36 .

6.314 Let $T_{i}$ be any point of $\Sigma$. Then $T_{i} \cup W$ and $T_{i} \cup Y$ are disconnected lines.

Proof: Suppose the flat $T_{i} \cup Y$ is connected (fig. 6E). There is a path $R_{0}$ from $Y$ to $T_{i}$ on $T_{i} \cup Y$, by 4.34 , which is off $C$. Similarly there is a path $R_{1}$ from $X^{\prime}$ to $T_{i}$ on $B$ and a path $R_{2}$ from $Z^{\prime}$ to $T_{i}$ on $B^{\prime}$, both paths being off $C$.

Now $\left(X^{\prime}, Y\right) R_{0} R_{1}^{-1}$ is a re-entrant path on the transversal $B \cup Y$ of dimension $n$, and $\left(Y, Z^{\prime}\right) R_{2} R_{0}^{-1}$ is a re-entrant path on the transversal $B^{\prime} \cup Y$ of dimension $n$. Both these paths are null-homotopic, by $H(n)$. We thus have

$$
\begin{aligned}
Q & \sim\left(W, X^{\prime}, Y, Z^{\prime}, W\right), \text { by } 6.311, \\
& =\left(W, X^{\prime}\right)\left(X^{\prime}, Y\right)\left(Y, Z^{\prime}\right)\left(Z^{\prime}, W\right) \\
& \sim\left(W, X^{\prime}\right) R_{1} R_{0}^{-1} R_{0} R_{2}^{-1}\left(Z^{\prime}, W\right), \text { by } 6.23, \\
& \sim\left(W, X^{\prime}\right) R_{1} R_{2}^{-1}\left(Z^{\prime}, W\right), \text { by } 6.22 .
\end{aligned}
$$

But the last path is on the $n$-flat $A$. Hence $Q \sim 0$ by $H(n)$, contrary to assumption.

We deduce that $Y \cup T_{i}$ has nontrivial separators $Y$ and $T_{i}$. Hence $Y$ and $T_{i}$ are the only points on it and the flat must be a disconnected line. (3.42 and 3.54.)

The transversal $B \cup Y$ of dimension $n$ is not on $W$. We can therefore repeat the construction for $B^{\prime}$, using $B \cup Y$ instead of $A$ and interchanging the roles of $Y$ and $W$. There results a transversal $B^{\prime \prime}$ of dimension $n-1$ on $B \cup Y$. Its pole on the line $U_{2} \cup Y$ is not in $C$ and its pole on the line $X^{\prime} \cup Y$ is in $C$. It meets $B$ in an $(n-2)$-flat $T^{\prime}$ analogous with $T$. 


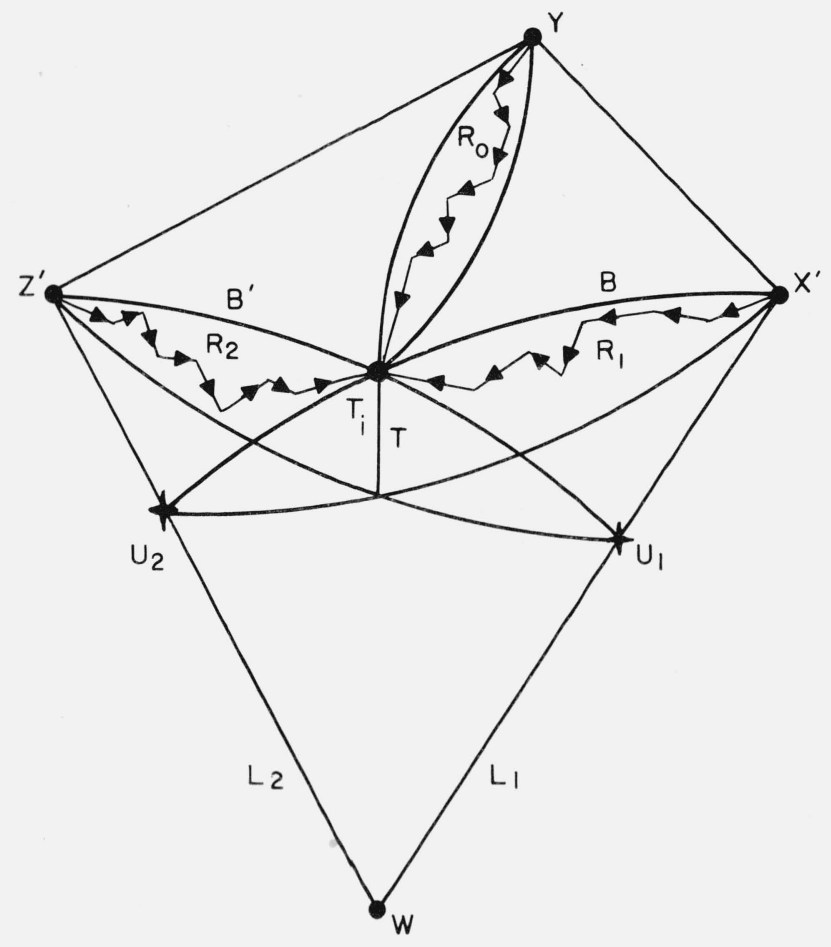

Figure 6E

In view of the foregoing argument we may assert that any point $T_{j}^{\prime}$ on $T^{\prime}$ which is not in $C$ has the property that $W \cup T_{j}^{r}$ is a disconnected line. In particular $B^{\prime \prime}$ has a point in common with the disconnected line $Y \cup T_{i}$, and this point can only be $T_{i}$. Hence $T_{i}$ is on $T^{\prime}$ and therefore $W \cup T_{i}$ is a disconnected line.

Let $K$ be a connected flat of $M$ which is on $F_{1}$ and $F(Q)$, and also on some point of $\Sigma$, and which has the least dimension consistent with these properties.
Clearly either $F(Q)$ or one of its subsets satisfies this definition.

\section{$6.315 d K=3$.}

Proof: We have

$$
n+1=d F(Q) \geqslant d K>d F_{1}=2,
$$

and so $d K \geqslant 3$. But $d T=n-2$. Hence, by 4.16 ,

$$
d\langle K \cap T\rangle \geqslant d K-3 \text {. }
$$

Choose a point $N$ on $\langle K \cap T\rangle$, taking $N$ in $C$ if this is possible. By 4.27 there is a connected $(d K-1)$-flat $K^{\prime}$ on $F_{1}$ and $K$, but not on $N$. By 4.14 and $4.16\left\langle K^{\prime} \cap T\right\rangle$ is a flat on $\langle K \cap T\rangle$ of dimension $d\langle K \cap T\rangle-1$.

All the points of $M$ on $\left\langle K^{\prime} \cap T\right\rangle$ belong to $C$, by the definition of $K$. By the choice of $N$ this implies that either $d\left\langle K^{\prime} \cap T\right\rangle=-1$ or $N$ is in $C$. But in the latter case all the points of $\langle K \cap T\rangle$ are in $C$, by 4.36 , which is contrary to the definition of $K$.

We deduce that $d\left\langle K^{\prime} \cap T\right\rangle=-1$ and therefore $d\langle K \cap T\rangle=0$. But we have seen that $d\langle K \cap T\rangle \geqslant d K$ -3 . The proposition follows.

\subsection{6. $n=2$.}

Proof: Suppose $n \geqslant 3$. Then $F_{2}$ is not on $K$, since $d F(Q)=d\left(F_{1} \cup F_{2}\right) \geqslant 4$, by 6.315 . By 4.27 there is a connected $n$-flat $K^{\prime \prime}$ on $F_{2}$ and $F(Q)$ but not on $T_{i}$. Write $F_{3}=\left\langle K^{\prime \prime} \cap K\right\rangle$. Then $F_{3}$ is a plane on $K$ and $W \cup Y$, by 4.14 and 4.16 .

By 4.27 there is a connected line $L$ on $K$ and $T_{i}$ which has no common point with the disconnected line $W \cup Y$. It meets $F_{1}$ and $F_{3}$ in distinct points $X_{1}$ and $X_{3}$, respectively, neither of which is $T_{i}$. (Fig. $6 \mathrm{~F}$.)

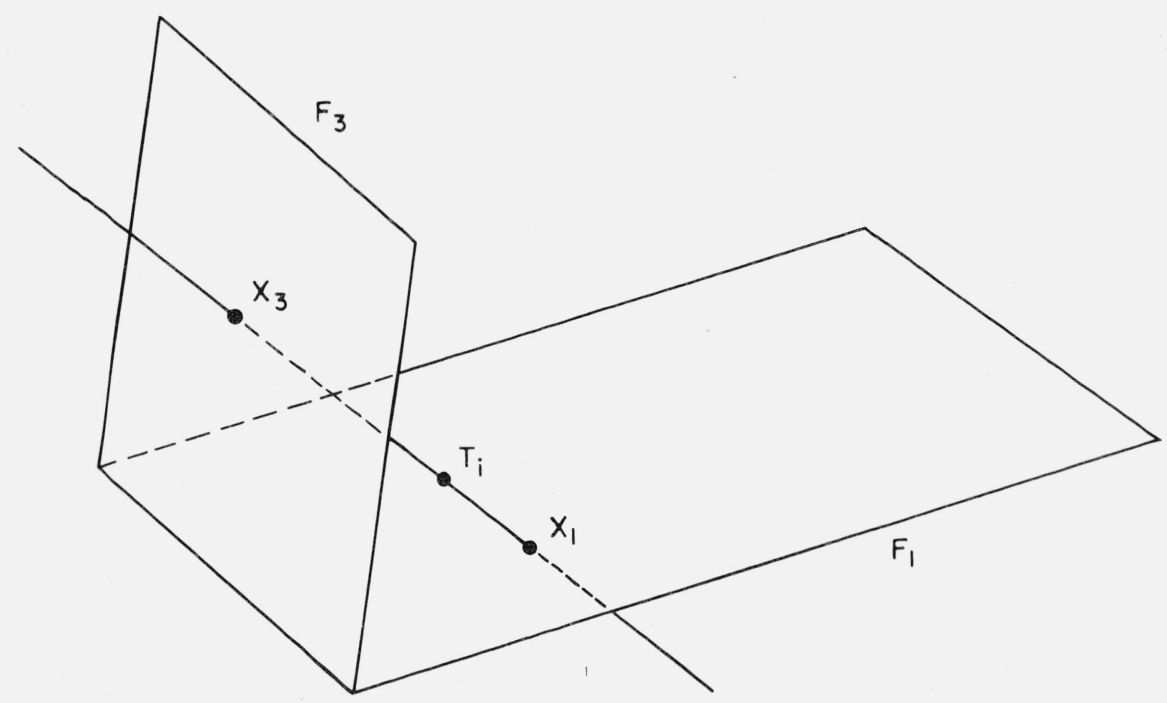

Figure 6F 
We note that $F_{3}=W \cup Y \cup X_{3}$, by 4.14. Moreover $X_{1} \cup W$ and $X_{1} \cup Y$ are connected lines, by 4.281. Hence $X_{1} \cap W$ and $X_{1} \cap Y$ are both non-null, by 4.22 . On the other hand $T_{i} \cup W$ and $T_{i} \cup Y$ are disconnected lines, by 6.314. Hence $T_{i} \cap W$ and $T_{i} \cap Y$ are null, by 4.22. But $L=X_{1} \cup T_{i}=X_{3} \cup T_{i}$, by 4.22. Hence $X_{3} \cap W$ and $X_{3} \cap Y$ are both non-null and therefore $F_{3}$ is connected by 3.48 .

By 4.35 there is a path $R$ from $Y$ to $W$ on $F_{3}$ which is off $C$. The re-entrant paths $(W, X, Y) R$ and $(Y$, $Z, W) R^{-1}$ are on $K$ and $K^{\prime \prime}$ respectively. They are thus null-homotopic, by $H(n)$. Hence

$$
Q=(W, X, Y)(Y, Z, W) \sim R^{-1} R \sim 0,
$$

by 6.22 and 6.23 .

Since $n \geqslant 2$ by the hypothesis of 6.31 we deduce, from this contradiction to our main assumption that $Q$ is not null-homotopic, that $n=2$.

It follows from this result that $d T=0$. Hence the flat $T$ is a point of $M$, identical with $T_{i}$. The three flats $W \cup Y, Y \cup T$ and $T \cup W$ are disconnected lines, by 6.314 . Hence $W \cup Y \cup T$ is not a connected plane, by 4.282 .

Any plane on the 3 -flat $F(Q)$ has a common point with each of the disconnected lines $W \cup Y, Y \cup T$ and $T \cup W$. It is therefore on one of these lines, by 4.23. Each line of $F(Q)$ is on a plane of $F(Q)$, by 4.14. Hence each such line is on $W, Y$, or $T$, by 4.171 .

6.317 There are just four connected lines on each of the planes $F_{1}$ and $F_{2}$, two being on $W$ and two on $Y$. Moreover each such line is on just three points, of which just one is in $C$.

Proof: Suppose one such line $L_{1}$ is on $k$ distinct points $X_{1}, X_{2}, \ldots, X_{k}$ other than $Y$ or $W$. Then $k>2$, by 4.23. Of course $L_{1}$ is on $Y$ or $W$.

Suppose $L_{1}$ is on $F_{1}$. By 4.27 there is a transversal $F$ of dimension 2 on $L_{1}$ and a transversal $B_{i}$ of dimension 1 on $F$ and $X_{i}$ for each $i . \quad(i=1,2,3)$. Then $F$ meets $F_{2}$ in a connected line $L_{2}$. The line $B_{i}$ is on $T$, since it is not on $W$ or $Y$. Hence $B_{i}=X_{i} \cup T$, and $B_{i}$ is uniquely determined for each $i$. Let $X_{i}^{\prime}$ be the common point of $B_{i}$ and $L_{2}$. Since $B_{1}=X_{i}^{\prime} \cup T$ the $k$ points $X_{1}{ }^{\prime}, X_{2}{ }^{\prime}, \ldots, X_{k}$ are all distinct. But at most one point on each of the lines $L_{1}$ and $L_{2}$ is in $C$, and each $B_{i}$ has at least one pole in $C$. Similar reasoning applies if $L_{1}$ is on $F_{2}$.

We deduce that each connected line on $F_{1}$ or $F_{2}$ is on just three points, and that just one of these points is in $C$.

There are two connected lines on $Y$ and two on $W$ in the plane $F_{1}$, by 4.26. Since the lines through $W$ (or $Y$ ) must meet those through $Y$ (or $W$ ) in distinct points it follows from the preceding result that there are just two such lines on $Y$ and just two on $W$. The same result holds for $F_{2}$.

6.318 Each of the disconnected lines $W \cup Y, Y \cup T$ and $T \cap W$ is on just two connected planes of $F(Q)$.

Proof: Let $L^{\prime}$ and $L^{\prime \prime}$ be the two connected lines on $W$ and $F_{1}$. (6.317). Each is on a transversal of dimension 2 , by 4.27 , and both these transversals are connected planes on $T \cup W$. There is no other con- nected plane on $T \cup W$ since each such plane would meet $F_{1}$ in a connected line through $W$. (We have shown that $T \cup W \cup Y$ is not a connected plane.) A similar argument applies for $T \cup Y$.

Now suppose there is a connected plane $F_{3}$, distinct from $F_{1}$ and $F_{2}$, on $W \cup Y$. We note that $F_{3}$ is not on $T$, since $W \cup Y \cup T$ is not a connected plane.

Let $U_{1}$ be a point of $C$ on $F_{1}$. By 4.27 it is on a transversal $B$ of dimension 1 . This must be on $T$. Let it meet $F_{1}, F_{2}$, and $F_{3}$ in $U_{1}, U_{2}$, and $U_{3}$ respectively. Similarly let $V_{2}$ be a point of $C$ on $F_{2}$. It is on a transversal $B^{\prime}$ of dimension $1 . B^{\prime}$ is on $T$ and meets $F_{1}, F_{2}$, and $F_{3}$ in distinct points $V_{1}, V_{2}$, and $V_{3}$ respectively. Since $T$ is not in $C$ we deduce that the points $U_{2}, U_{3}, V_{1}$, and $V_{3}$ are not in $C$. (Fig. 6G.)

We have

$$
\begin{aligned}
\left(W, V_{1}, Y\right) & \sim\left(W, V_{1}, V_{3}, W\right)\left(W, V_{3}, V_{1}, Y\right) \\
& \sim\left(W, V_{1}, V_{3}, W\right)\left(W, V_{3}, Y\right)\left(Y, V_{3}, V_{1}, Y\right) \\
& \sim\left(W, V_{3}, Y\right),
\end{aligned}
$$

by 6.21 and 6.22 , with the help of $H(n)=H(2)$. Similarly

$$
\left(W, U_{2}, Y\right) \sim\left(W, U_{3}, Y\right)
$$

\section{Hence}

$Q \sim\left(W, V_{1}, Y, U_{2}, W\right)$, by $6.311, \sim\left(W, V_{3}, Y, U_{3}, W\right)$, by the preceding results,

$\sim 0$, by $H(2)$.

But this is contrary to assumption. The proposition follows.

We observe that our figure on $F(Q)$ is a box with radiants $Y, W$, and $T$. We therefore revert to the notation of section 6.1. We put $Y=Z_{1}, W=Z_{2}$ and $T=Z_{3}$. We also identify $P_{3}$ with $F_{1}$ and $P_{6}$ with

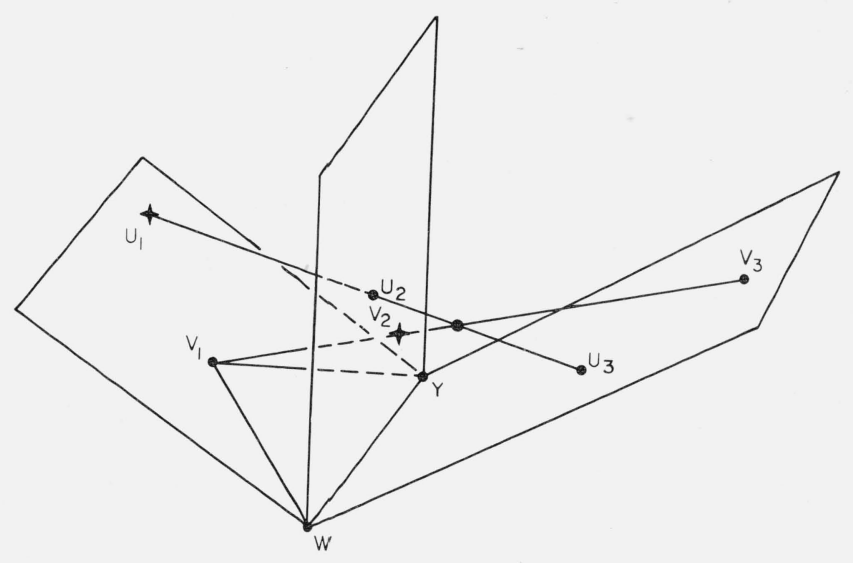

Figure 6G 
$F_{2}$. Each connected line is on $Y, W$, or $T$, and is thus on at most one point of $C$. But each connected line on $P_{3}$ or $P_{6}$ is on one point of $C$, by 6.317 . We may therefore suppose the points of $C$ to be as shown in figure $6 \mathrm{~B}$.

This implies that $Q$ is an elementary re-entrant path of the fourth kind. Thus $Q \sim 0$, contrary to assumption. This contradiction establishes the lemma 6.31.

\subsection{Homotopy Theorem}

We now turn to the proof of the main theorem of the chapter.

6.41 Every re-entrant path of $M$ which is off $C$ is nullhomotopic

Proof: We proceed by induction. We observe first that $H(0)$ is trivially true. We assume as an inductive hypothesis that $H(n)$ is true for some nonnegative integer $n$, and we try to deduce the truth of $H(n+1)$.

Let $P$ be any path of $M$ off $C$ which is re-entrant and has dimension $n+1$. Choose a connected $n$-flat $J$ of $M$ which is on $F(P)$ and the origin $X_{0}$ of $P$, as is possible by 4.25 and 6.24 .

Let $R$ be any re-entrant path on $F(P)$ with origin $X_{0}$. Write

$$
R=\left(X_{0}, X_{1}, X_{2}, \ldots, X_{m}, X_{0}\right) .
$$

We write $u(R)$ for the number of terms of $R$, counting repetitions, which are not on $J$. If $u(R)>0$ we denote by $X_{i}$ the first term of $R$ which is not on $J$. We then write $v(R)$ for the dimension of the connected flat $X_{i-1} \cup X_{i} \cup X_{i+1}$, taking $X_{m+1}=X_{0}$ if $i=m$. If $u(R)=0$ we write $v(R)=0$.

We may suppose $R$ chosen so as to satisfy the following conditions

(i) $R \sim P$

(ii) $u(R)$ has the least value consistent with (i), (ii).

(iii) $v(R)$ has the least value consistent with (i) and

We write $F=X_{i-1} \cup X_{i} \cup X_{i+1}$. We also write $R$ in the form

$$
R_{1}\left(X_{i-1}, X_{i}, X_{i+1}\right) R_{2}
$$

so that $R_{1}$ is a path on $J$.

We assume $u(R)>0$. This implies $v(R)>0$. We consider separately the cases $v(R)=1, v(R)=2$ and $v(R) \geqslant 3$.

Case $I \quad v(R)=1$.

In this case $F$ is a connected line. If $X_{i+1}=X_{i-1}$ we have $P \sim R \sim R_{1} R_{2}$, by an elementary deformation of the first kind. Since $u\left(R_{1} R_{2}\right)<u(R)$ this is contrary to the choice of $R$.

If $X_{i+1} \neq X_{i-1}$, then

$$
\left(X_{i-1}, X_{i}, X_{i+1}, X_{i-1}\right)
$$

is an elementary re-entrant path of the second kind.

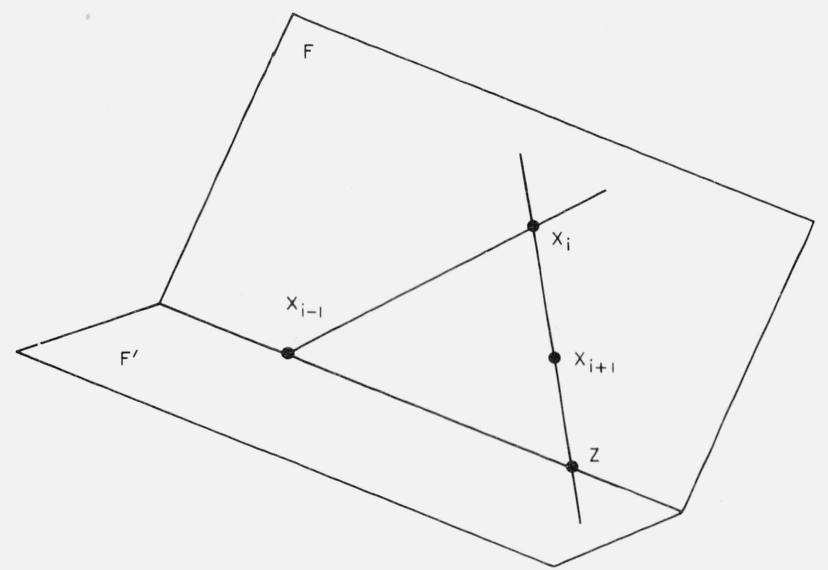

Figure 6H

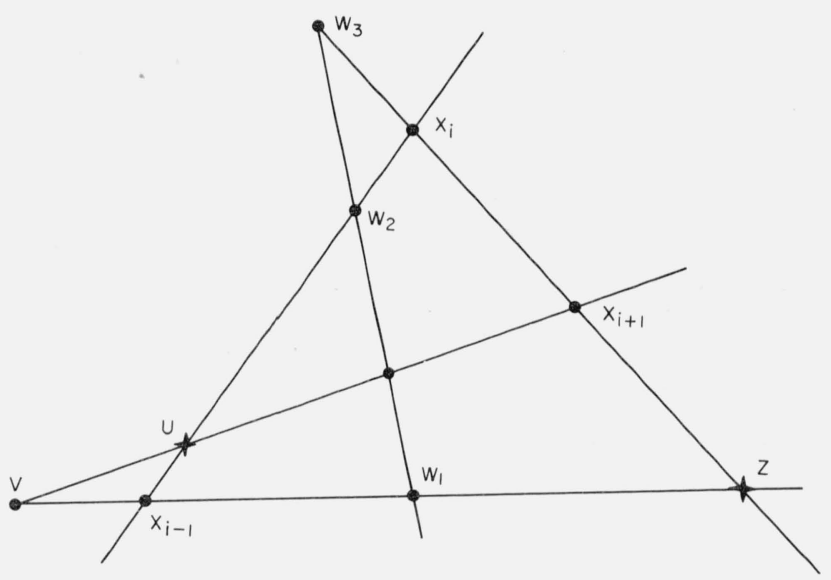

Figure 6I

Hence

$$
\left.P \sim R \sim R_{1}\left(X_{i-1}, X_{i+1}\right) R_{2}\right)
$$

by 6.23 . But this is impossible since

$$
u\left(R_{1}\left(X_{i-1}, X_{i+1}\right) R_{2}\right)<u(R) .
$$

Case II: $v(R)=2$.

$F$ is now a connected plane on $F(P)$. It meets $J$ in a line $L$, by 4.14 and 4.16 . Let $Z$ be the point of intersection of the lines $L$ and $X_{i} \cup X_{i+1}$ on $F$. (Figs. $6 \mathrm{H}$ and 6I.)

Suppose $Z$ is not in $C$, as in figure $6 \mathrm{H}$. We define $Q$ as the degenerate path $(Z)$ if $Z=X_{i+1}$, and as the path $\left(Z, X_{i+1}\right)$ otherwise. Then

$$
\left(X_{i}, X_{i+1}\right) Q^{-1}\left(Z, X_{i}\right)
$$


is an elementary re-entrant path of the first or second kind. Hence

$$
R \sim R_{1}\left(X_{i-1}, X_{i}, Z\right) Q R_{2},
$$

by 6.23 . If $L$ is connected we have

$$
\left(X_{i-1}, X_{i}, Z, X_{i-1}\right) \sim 0,
$$

by an elementary deformation of the second kind, and so

$$
R \sim R_{1}\left(X_{i-1}, Z\right) Q R_{2},
$$

by 6.23 .

If $L$ is not connected it is on a connected plane $F^{\prime}$ contained in $J$, by 4.28 . We can find a connected line $L^{\prime}$ on $X_{i-1}$ and $F^{\prime}$ by 4.26 , and a point $T$ on $L^{\prime}$ distinct from $X_{i-1}$ and not in $C$, by 4.23 . The flats $T \cup X_{i-1}$ and $T \cup Z$ are connected lines, by 4.282 . Hence

$$
\left(X_{i-1}, X_{i}, Z, T, X_{i-1}\right) \sim 0,
$$

by 6.31 . So by 6.23 we have

$$
R \sim R_{1}\left(X_{i-1}, T, Z\right) Q R_{2} .
$$

Thus, whether or not $L$ is connected, we have

$$
R \sim R_{3} Q R_{2},
$$

where $R_{3}$ is on $J$. But this is contrary to the choice of $R$, since $u\left(R_{3} Q R_{2}\right)<u(R)$.

We may now suppose $Z \epsilon C$, as shown in figure 6I. In this case there is a connected line $L^{\prime}$ other than $X_{i} \cup X_{i+1}$ on $X_{i+1}$ and $F$, by 4.26 . If $L^{\prime}$ is on $X_{i-1}$ then $\left(X_{i-1}, X_{i}, X_{i+1}, X_{i-1}\right)$ is an elementary re-entrant path of the second kind. We therefore have

$$
R \sim R_{1}\left(X_{i-1}, X_{i+1}\right) R_{2},=R^{\prime} \text { say, }
$$

by 6.23 . This is contrary to the choice of $R$, since $u\left(R^{\prime}\right)<u(R)$. We deduce that in fact $L^{\prime}$ is not on $X_{i-1}$. It therefore meets the lines $X_{i-1} \cup X_{i}$ and $L$ in distinct points $U$ and $V$ respectively. Since $Z$ is in $C$ and $X_{i-1}$ is not, the point $V$ is not in $C$.

Suppose $U$ is not in $C$, contrary to the indication given in figure 6I. Using 6.23 with elementary re-entrant paths of the second kind we have

$$
\begin{aligned}
R & \sim R_{1}\left(X_{i-1}, U, X_{i}, X_{i+1}\right) R_{2} \\
& \sim R_{1}\left(X_{i-1}, V, U, X_{i+1}\right) R_{2} \\
& \sim R_{1}\left(X_{i-1}, V, X_{i+1}\right) R_{2}, \quad=R^{\prime} \text { say. }
\end{aligned}
$$

But then $u\left(R^{\prime}\right)<u(R)$, contrary to the choice of $R$.

We deduce that $U$ is in $C$, as indicated in figure $6 \mathrm{I}$. In this case it may happen that each connected line on $F$ is on either $U$ or $Z$. Then $\left(X_{i-1}, X_{i}, X_{i+1}, V, X_{i-1}\right)$ is an elementary re-entrant path of the third kind. Using 6.23 we deduce that

$$
R \sim R\left(X_{i-1}, V, X_{i+1}\right) R_{2},
$$

which is impossible, as before.

It follows that there is a connected line $L^{\prime \prime}$ on $F$ which is not on $U$ or $Z$. Let it cut the lines $L, X_{i-1} \cup X_{i}$ and $X_{i} \cup X_{i+1}$ in the points $W_{1}, W_{2}$, and $W_{3}$ respectively. These points are distinct from $U$ and $Z$ and are therefore not in $C$.

If $L^{\prime \prime}$ is on $X_{i+1}$ we can substitute it for $L^{\prime}$ in the preceding argument. This reduces the problem to the case in which $U$ is not in $C$, and so yields a contradiction. We may accordingly suppose that $L^{\prime \prime}$ is not on $X_{i+1}$.

If $L^{\prime \prime}$ is on $X_{i}$ it meets $L$ in a point $W_{1}$ distinct from $X_{i-1}$ and $Z$.

Writing

$$
R^{\prime}=R_{1}\left(X_{i-1}, W_{1}, X_{i}, X_{i+1}\right) R_{2},
$$

we then have $R \sim R^{\prime}$, by an elementary deformation of the second kind. If $L^{\prime \prime}$ is not on $X_{i}$ then the points $W_{2}, W_{3}, X_{i}, X_{i+1}$, and $Z$ are all distinct. If $L^{\prime \prime}$ is then on $X_{i-1}$ we write

$$
R^{\prime}=R_{1}\left(X_{i-1}, W_{3}, X_{i+1}\right) R_{2},
$$

and have $R \sim R^{\prime}$, by elementary deformations. If however $L^{\prime \prime}$ is not on $X_{i-1}$ the points $X_{i-1}$ and $W_{2}$ are distinct. We then write

$$
R^{\prime}=R_{1}\left(X_{i-1}, W_{1}, W_{3}, X_{i+1}\right) R_{2}
$$

and have

$$
\begin{aligned}
R & \sim R_{1}\left(X_{i-1}, W_{2}, X_{i}, X_{i+1}\right) R_{2} \\
& \sim R_{1}\left(X_{i-1}, W_{1}, W_{2}, W_{3}, X_{i}, X_{i+1}\right) R_{2} \\
& \sim R^{\prime},
\end{aligned}
$$

by 6.23 . For each of these three possibilities we have

$$
R \sim R^{\prime}, u\left(R^{\prime}\right)=u(R), v\left(R^{\prime}\right)=v(R)=2 .
$$

Hence we may replace $R$ by $R^{\prime}$ in the preceding argument. This reduces the problem to the case in which $U$ is not in $C$, and so yields a contradiction.

This completes the analysis of Case II.

Case III. $v(R) \geqslant 3$.

There is a connected plane $K$ on $F$ and the line $X_{i-1} \cup X_{1}$. This plane meets $J$ in a line $L$. Choose a point $N$, distinct from $X_{i-1}$, on $L$ and if possible in $C$. By 4.27 there is a connected $(v(R)-1)$-flat $F^{\prime}$ on $X_{i} \cup X_{i+1}$ and $F$, but not on $N$. Now $F^{\prime}$ is not on $X_{i-1}$, for otherwise we would have $F=X_{i-1} \cup X_{i} \cup X_{i+1} \subseteq F^{\prime}$. Hence $F^{\prime}$ meets $L$ in a point $N^{\prime}$ distinct from $N$ and $\bar{X}_{i-1}$. It follows that $L$ is connected, by 4.23 , and that $N^{\prime}$ is not in $C$. (See fig. 6J.) 


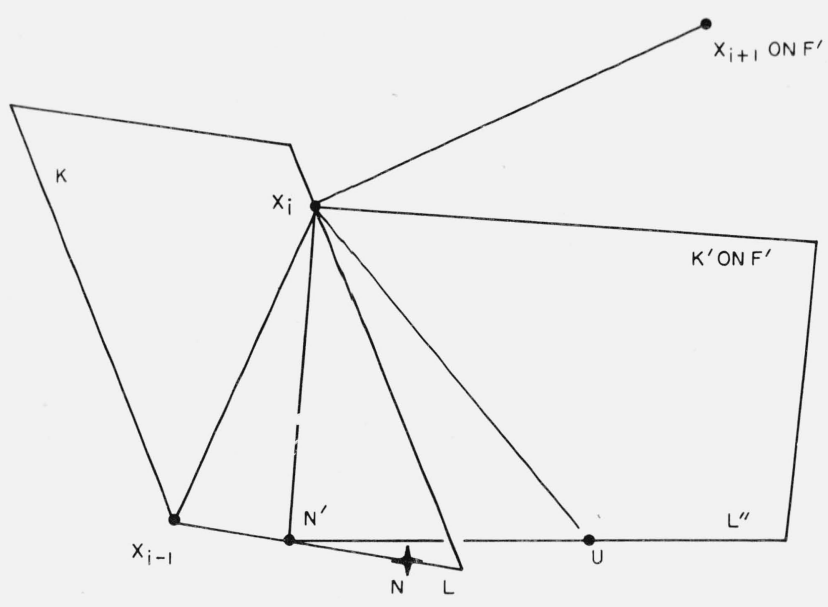

Figure 6J

\subsection{Special Cases}

When $C$ is null there are only two kinds of elementary re-entrant path to consider, the first and the second. The homotopy theorem then tells us that every re-entrant path in a matroid can be deformed into a degenerate one by a sequence of operations each of which is confined to a single line or plane.

The homotopy theorem can of course be applied to the polygon-matroid of a graph $G$. But care must be taken to distinguish between the paths of $P(G)$ and those of $G$ itself. A path in $P(G)$ corresponds to a sequence of polygons in $G$ such that the union of two consecutive polygons is a nonseparable subgraph $H$ such that $P(H)$ has rank 2 . It can be shown that these conditions on $H$ imply that $H$ is a " $\theta$-graph", made up of three arcs such that any two have both ends but no other edge or vertex in common.

The homotopy theorem with $C$ null asserts that a re-entrant sequence of polygons of the above kind can be reduced to a degenerate sequence by operations each of which is confined to a single polygon or $\theta$-graph of $G$.

\section{Characterization of Regular Matroids}

$$
R^{\prime}=R_{1}\left(X_{i-1}, N^{\prime}, X_{i}, X_{i+1}\right) R_{2},
$$

and have $R^{\prime} \sim R$ by 6.23 . If $L^{\prime}$ is not connected it is on a connected plane $K^{\prime}$ on $F^{\prime}$, by 4.28 . This meets $J$ in a connected line $L^{\prime \prime}$ on $N^{\prime}$, by 4.282 . We can find a point $U$ on $L^{\prime \prime}$ distinct from $N^{\prime}$ and not in $C$. The flat $U \cup X_{i}$ is a connected line, by 4.282 . We now have

$$
\left(N^{\prime}, U, X_{i}, X_{i-1}, N^{\prime}\right) \sim 0,
$$

by 6.31 . In this case we write

$$
R^{\prime}=R_{1}\left(X_{i-1}, N^{\prime}, U, X_{i}, X_{i+1}\right) R_{2} .
$$

We then have

$$
\begin{aligned}
R^{\prime} & \sim R_{1}\left(X_{i-1}, N^{\prime}, U, X_{i}, X_{i-1}, N^{\prime}, X_{i-1}, X_{i}, X_{i+1}\right) R_{2} \\
& \sim R_{1}\left(X_{i-1}, N^{\prime}, X_{i-1}, X_{i}, X_{i+1}\right) R_{2} \\
& \sim R_{1}\left(X_{i-1}, X_{i}, X_{i+1}\right) R_{2} \sim R,
\end{aligned}
$$

by 6.23 and an elementary deformation.

So, whether $L^{\prime}$ is connected or not, we have

$$
R^{\prime} \sim R, \quad u\left(R^{\prime}\right)=u(R), \quad v\left(R^{\prime}\right)<v(R) .
$$

But this is contrary to the definition of $R$.

From the above analysis of the hypothesis $u(R)>0$ we deduce that in fact $u(R)=0$. Hence $R$ is on $J$ and has dimension $\leqslant n$, and therefore $R \sim 0$, by $H(n)$. Hence $P \sim 0$.

We have now shown that $H(n+1)$ is deducible from $H(n)$. Hence the proposition $H(n)$ is true for every non-negative integer $n$ (since $H(0)$ is true), by induction. Thus our theorem holds.

\subsection{Some Preliminary Observations}

In this chapter we resume the discussion of regular chain-groups and matrioids which was broken off at the end of section 5.5. Our task is to show that a binary matroid is regular if it has no minor of Type $\mathrm{BI}$ or BII.

We first note some simple theorems which will be helpful in the discussion.

7.11 Let $S$ be an $(n-1)$-flat on an $n$-flat $T$ of a matroid $M$. Let $a$ be any cell of $T-S$. Then $\langle T-\{a\}\rangle=S$.

This follows from 4.13 and 4.14.

7.12 Let $X, Y$ and $Z$ be distinct collinear points of $a$ binary matroid $M$. Then $X+Y+Z$ is null.

A proof of this theorem is contained in the proof of 5.35 .

7.13 Let $S$ be an $n$-flat on an $(n+2)$-flat $T$ of a binary matroid $M$, where $n \geqslant 0$. Then there are at most three $(n+1)$-flats of $M$ which are on both $S$ and $T$.

Proof: Suppose there are $k$ such $(n+1)$-flats.

By 4.27 there is a line $L$ on $T$ which has no common point with $S$. The $k(n+1)$-flats meet $L$ in $k$ distinct points. Hence $\mathrm{k} \leqslant 3$, by 5.35 and 4.23 .

\subsection{Fano Configuration}

We can define a Fano configuration in a binary matroid $M$ as a figure made up of a plane $P$ of $M$, seven distinct lines on $P$, and the points of intersection of these seven lines.

Suppose $F$ is such a configuration. Any line $L$ of $F$ is on at most three points, by 5.35 , and each of these is on at most two other lines on $P$. But each line on $P$ must have a common point with $L$. We deduce that each line of $F$ is on three distinct points of $F$, each of which is on exactly two other lines of $F$. Hence $F$ 
has just seven points. Moreover there can be no eighth line on $P$, and therefore there is no eighth point on $P$, by 4.25 and 5.35 .

We enumerate the seven lines as $L_{1}, L_{2}, \ldots, L_{7}$. We denote the point of intersection, if any, of three lines $L_{i}, L_{j}$, and $L_{k}$ by $X_{i j k}$.

We may assume a point $X_{135}$ on $L_{1}, L_{3}$, and $L_{5}$. The line $L_{7}$, which is not on $X_{135}$, meets $L_{1}, L_{3}$, and $L_{5}$ in points we may label $X_{147}, X_{367}$, and $X_{257}$ respectively. The lines $L_{2}$ and $L_{4}$ each meet $L_{3}$ in its point other than $X_{135}$ and $X_{367}$, which point is therefore $X_{234}$. Similarly the remaining points of the figure are $X_{456}$ and $X_{126}$.

A Fano configuration is shown in figure $7 \mathrm{~A}$, in which one of the seven "lines" is indicated by a curve.

The seven lines are connected, by 4.23 , and so $P$ is connected, by 3.48 .

7.21 If a binary matroid $M$ includes a Fano configuration, then it has a minor of Type BI.

Proof: Let $P$ be the plane of the Fano configuration. There is a binary chain-group $N$ such that $M(N)$ $=M \times P$, by 5.31 .

Consider the chains $f, g$, and $h$ on $P$ over $G F(2)$ whose domains are $X_{126}, X_{234}$, and $X_{456}$ respectively. They are linearly independent chains of $N$ since $X_{126}$ is not on $L_{4}$, that is not contained in $X_{234} \cup X_{456}$. Moreover they generate the chains corresponding to all the atoms of $M \times P$, by 7.12. Hence their representative vectors can be taken as the three rows of a representative matrix $K$ of $N$, by 5.32 .

Since $P$ is a union of atoms of $M$ the matrix $K$ has no zero column. If it has two equal columns corresponding to cells $a$ and $b$ it is. clear that an atom of $M \times P$ contains $a$ if and only if it contains $b$. But then $\langle P-\{a\}\rangle=\langle P-\{b\}\rangle$.

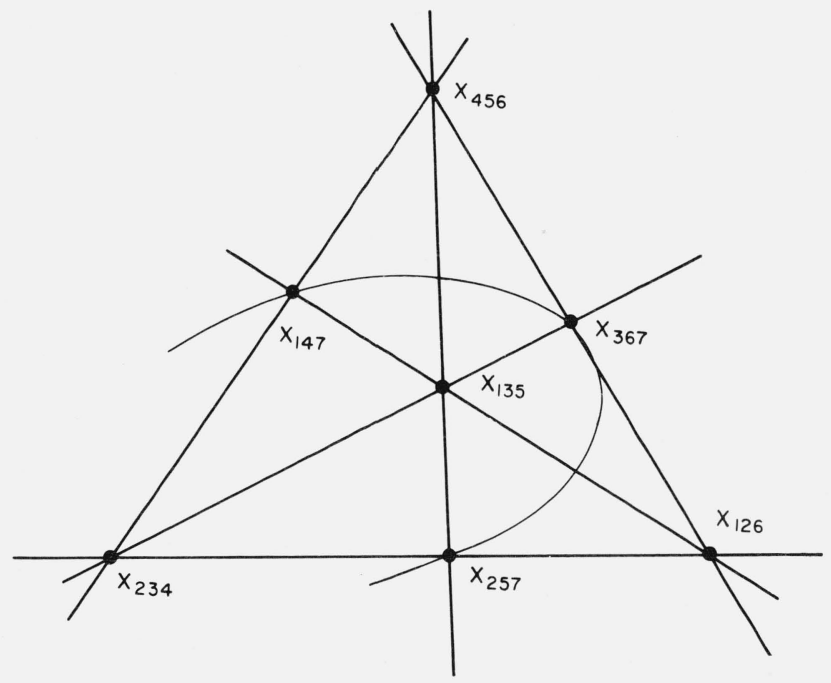

FIGURE 7A
Since there are seven distinct lines on $P$ it follows from 7.11 that all the seven distinct nonzero 3 -vectors over $G F(2)$ occur as columns of $K$. Hence, there exists $S \subseteq P$ such that $K(S)$ is the first matrix of section 5.5, apart from a permutation of columns. But then $(M \times P) \cdot S$ is a matroid of Type BI, by 5.21. Since $(M \times P) \cdot S$ is a minor of $M$ the theorem follows.

\subsection{Heptahedron}

We use the term "heptahedron" to denote a figure on a 3 -space $F$ of a binary matroid $M$, defined by seven distinct planes such that no three are on a common line. We include in the figure the 21 lines of intersection of the seven planes, and those points of $F$ which are common to three or more of the seven planes.

We enumerate the seven planes as $P_{1}, P_{2}, \ldots$. , $P_{7}$, and write $L_{i j}$ for the line of intersection of $P_{i}$ and $P_{j}$, taking $0 \leqslant i<j \leqslant 7$.

A point on $L_{i j}$ is on at most three lines of $P_{i}$, by 7.13. On the other hand there are exactly six lines of the heptahedron on $P_{i}$. It follows that each of the lines $L_{i j}$ is on exactly three points of the figure, that one of these points is on exactly three of the seven planes, and that each of the two other points is on exactly four of them (5.35). Hence each of the lines $L_{i j}$ is connected. It follows that the planes $P_{i}$ and the flat $F$ are connected.

We write $X_{i j k}$ for a point which is on just three planes $P_{i}, P_{j}$, and $P_{k}$ of the heptahedron, and $X_{i j k l}$ for a point on just four such planes $P_{i}, P_{j}, P_{k}$, and $P_{l}$. Each point of the first kind is on exactly three lines of the heptahedron, and each point of the second kind on exactly six. It follows that the figure has just seven points of each kind.

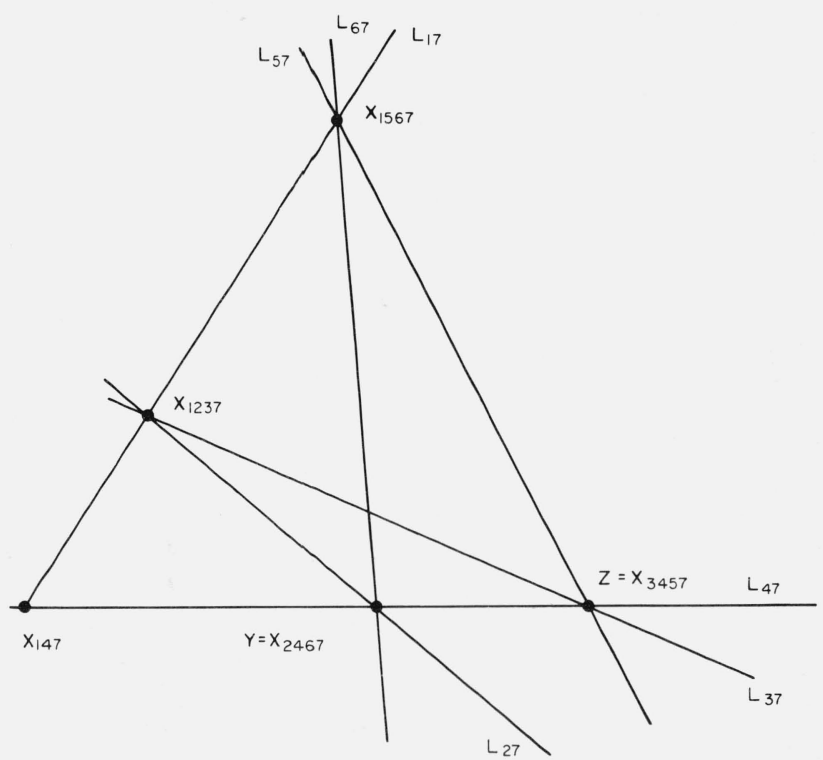

FiguRE 7B 
We may assume $L_{17}$ to be on $X_{147}, X_{1237}$, and $X_{1567}$. The line $L_{47}$ is on two points $Y$ and $Z$ distinct from $X_{147}$. On $P_{7}$ each of these is on three lines of the heptahedron meeting $L_{17}$ in three distinct points. We may therefore suppose $Y$ to be on $L_{27}$ and $L_{67}$ while $Z$ is on $L_{37}$ and $L_{57}$. Then $Y=X_{2467}$ and $Z=X_{3457}$. (Fig. 7B.)

The remaining points of the heptahedron on $P_{7}$ are $X_{367}=\left\langle L_{37} \cap L_{67}\right\rangle$ and $X_{257}=\left\langle L_{27} \cap L_{57}\right\rangle$.

The lines $L_{13}$ and $L_{14}$ on $P_{1}$ meet in a point $X_{134 x}$, since $L_{14}$ is on $X_{147}$. Since no three planes of the heptahedron are on a common line this point cannot have three suffices in common with any of the points on $P_{7}$. It is therefore $X_{1346}$. But $X_{1346}$ is on the lines $L_{13}, L_{14}, L_{16}, L_{34}, L_{36}$, and $L_{46}$. These meet $P_{7}$ in $X_{1237}$, $X_{147}, X_{1567}, X_{3457}, X_{367}$, and $X_{2467}$ respectively. The third points on these lines are therefore $X_{135}, X_{1245}$, $X_{126}, X_{234}, X_{2356}$, and $X_{456}$.

We have now accounted for all the points of the figure. Those of the first kind are

$$
X_{126}, X_{135}, X_{147}, X_{234}, X_{257}, X_{367}, X_{456} \text {, }
$$

and those of the second kind are

$$
X_{1237}, X_{1245}, X_{1346}, X_{1567}, X_{2356}, X_{2467}, X_{3457} .
$$

A heptahedron can be constructed from the box of figure 6B. To do this we replace the three disconnected lines by connected ones, and we imagine a seventh plane to pass through the points of $C$ and to cut the lines $Z_{i} Z_{j}$ in their third points. Since any two points of $C$ determine a unique plane of the box it is clear that no line of the resulting figure is on three of the seven planes. The notation of figure $6 \mathrm{~B}$ is not that used above.

7.31 If a binary matroid $M$ includes a heptahedron, then it has a minor of Type BII.

Proof: We use for the heptahedron the notation set out above. There is a binary chain-group $N$ such that $M(N)=M \times F$, by 5.31: The rank of $M(N)$ is 4 .

Let $f, g, h$, and $j$ be the chains on $F$ over $G F(2)$ whose domains are $X_{234}, X_{1346}, X_{1245}$, and $X_{1237}$ respectively. Any three of these atoms define a plane which is not on the fourth, so the four chains are linearly independent. Using 7.12 we can verify that they generate the chains corresponding to the atoms of $M(N)$, and therefore all the chains of $N$ by 5.32 . Hence their representative vectors can be taken as the four rows of a representative matrix $K$ of $N$.

For each $P_{i}$ we select a cell $a_{i} \epsilon F-P_{i}$. We then have $P_{i}=\left\langle F-\left\{\mathrm{a}_{i}\right\}\right\rangle$, by 7.11 . We write $S=\left\{a_{1}\right.$, $\left.a_{2}, \ldots, a_{7}\right\}$. We further suppose that the enumeration of the cells of $F$, used in defining $K$, is such that the columns representing the cells $a_{i}$ occur in the order of their suffices.

Now a plane $P_{i}$ passes through a point $X$ of the heptahedron if and only if $i$ is one of the suffices of $X$. Moreover this happens if and only if $a_{i}$ is not a cell of $X$. We can therefore evaluate the matrix

\begin{tabular}{|c|c|c|c|c|c|c|}
\hline & 0 & 0 & 0 & 1 & & 1 \\
\hline & 1 & 0 & 0 & 1 & 0 & \\
\hline & 0 & 1 & 0 & 0 & 1 & \\
\hline & 0 & 0 & 1 & 1 & 1 & \\
\hline
\end{tabular}

$K(S)$ as

This is a representative matrix of the minor $M_{1}=$ $(M \times F) \cdot S$ of $M$, by 5.21. But then $M_{1}$ * is a matroid of Type BI, by 5.23 . Thus $M_{1}$ is of Type BII, and the theorem follows.

Theorems 7.21 and 7.31 have simple converses. It is easily verified that a matroid of Type BI or BII has a Fano configuration or heptahedron on its plane or 3-space. If the matroid is a minor $(M \times S) \cdot T$ of a larger matroid $M$, a corresponding configuration must occur on any carrier $Z$ of $(M \times S) \cdot T$ in $M \times S$. (See section 4.4). The configuration then occurs on the flat $\langle Z\rangle$ of $M$.

\subsection{Condition for a Regular Matroid}

We devote this section to a proof of the following theorem.

7.41 A binary matroid $M$ on a set $E$ is regular if it includes no Fano configuration and no heptahedron.

Proof: Assume the theorem false. Then there exists a matroid $M$, on a set $E$, which is binary and includes no Fano configuration or heptahedron, but which is not regular. Choose such a matroid $M$ so that $|E|$ has the least possible value.

Clearly $|E| \geqslant 1$. Choose $a \in E$ and write $M^{\prime}=$ $M \cdot(E-\{a\})$. Then $M^{\prime}$ has a carrier $Z$ in $M$, and $Z$ is either $E$ or $E-\{a\}$. By section 4.4 there is a $1-1$ mapping $\theta$ of the set of flats of $M^{\prime}$ onto a subset of the set of flats of $M$ which preserves dimension and geometrical incidence.

As in the case of 6.31 we arrive at a proof of 7.41 through a sequence of subsidiary theorems.

$7.411 M^{\prime}$ is regular.

Proof: $M^{\prime}$ is binary, by 5.31. It includes no Fano configuration or heptahedron, for otherwise $\theta$ would induce another such figure in $M$. Hence $M^{\prime}$ is regular, by the choice of $M$.

By 7.411 there is a regular chain-group $N^{\prime}$ on $E-\{a\}$ such that $M^{\prime}=M\left(N^{\prime}\right)$. For each atom $X$ of $M^{\prime}$ we select a primitive chain $g(X)$ of $N^{\prime}$ such that $\|g(X)\|=X$. For each $b \epsilon E-\{a\}$ we denote the coefficient of $b$ in $g(X)$ by $s(X, b)$. Thus $s(X, b)$ is 1 or -1 if $b \in X$, and is zero otherwise.

Let $C$ denote the class of all points $T$ of $M^{\prime}$ such that $a \notin \theta T$.

$7.412 C$ is a linear subclass of $M^{\prime}$.

Proof: Suppose $X, Y$, and $Z$ are distinct points of $M^{\prime}$ on a line $L$ of $M^{\prime}$, and that $X$ and $Y$ belong to $C$. 
Then $\theta X, \theta Y$, and $\theta Z$ are distinct points of $M$ on $\theta L$, and $\theta L$ is a line of $M$. But now $a \notin \theta X \cup \theta Y=\theta L$, by 4.22 , and therefore $a \notin \theta Z$. Hence $Z \epsilon C$. The proposition follows.

Let $X$ and $Y$ be any two distinct points of $M^{\prime}$, not in $C$, which are on the same connected line $L$ of $M^{\prime}$. By 4.22 there exists a cell $d \epsilon X \cap Y$. We now write

$$
t(X, Y)=s(X, d) s(Y, d) .
$$

7.413 If $X$ and $Y$ satisfy the conditions just stated, then $t(X, Y)$ is uniquely determined.

Proof: Suppose this proposition is false for some $X$ and $Y$. Then there are distinct cells $d$ and $e$ of $X \cap Y$ such that

$$
s(X, d) s(Y, d)=-s(X, e) s(Y, e)
$$

Without loss of generality we may assume that

$$
\begin{aligned}
& s(X, d)+s(Y, d)=0, \\
& s(X, e)+s(Y, e)= \pm 2 .
\end{aligned}
$$

The third point $Z$ on $L$ is the $\bmod 2$ sum of $X$ and $Y$, by Hence we have

$$
Z \subset\|g(X)+g(Y)\| \subset L,
$$

since $e$ is in $\|g(X)+g(Y)\|$ but not $Z$, and $d$ is in $L$ but not $\|g(X)+g(Y)\|$. But $\|g(X)+g(Y)\|$ is a flat of $M^{\prime}$, by 5.43 . Hence this result implies that $d L \geqslant 2$, by 4.14 , which is contrary to the definition of a line. The proposition follows.

If $R=\left(X_{1}, \ldots, X_{k}\right)$ is any nondegenerate path off $C$ in $M^{\prime}$ we write

$$
u(R)=\prod_{i=1}^{k-1} t\left(X_{i}, X_{i+1}\right)
$$

7.414 If $R$ is any nondegenerate re-entrant path off $C$ on $M^{\prime}$, then $u(R)=1$.

Proof: We first note that by 6.41 , there is a positive integer $m(R)$ which is the least number of elementary deformations, with respect to $C$, required to convert $R$ into a degenerate path.

Assume the proposition false. Then we can find a nondegenerate re-entrant path $Q$ off $C$ on $M^{\prime}$ such that $u(Q)=-1$. and $m(Q)$ has the least value consistent with this condition.

If $m(Q)>1$ we can write either $Q=Q_{1} U Q_{2}$ and $Q^{\prime}=Q_{1} Q_{2}$ or $Q^{\prime}=Q_{1} U Q_{2}$ and $Q=Q_{1} Q_{2}$, where $U$ is an elementary re-entrant path with respect to $C$ and $m\left(Q^{\prime}\right)=m(Q)-1>0$. In either case we have

$$
u(Q)=u(Q) \cdot u\left(Q^{\prime}\right)
$$

But this is impossible, since $u(Q)=u\left(Q^{\prime}\right)=1$ by the choice of $Q$. We deduce that in fact $m(Q)=1$. In other words $Q$ is an elementary re-entrant path witi. respect to $C$.

We must now consider four cases, one for each kind of elementary re-entrant path.

Case I. $Q$ is of the first kind.

We have $Q=(X, Y, X)$ for some points $X$ and $Y$. Hence

$$
u(Q)=\{t(X, Y)\}^{2}=1 .
$$

Case II. $Q$ is of the second kind.

In this case we have $Q=(X, Y, Z, X)$, where $X, Y$, and $Z$ are distinct points on the same line or plane of $M^{\prime}$.

If they are on the same line $L$ of $M^{\prime}$, then $\theta X, \theta Y$ and $\theta Z$ are distinct points on the line $\theta L$ of $M$. But $a \epsilon \theta X \cup \theta Y \cup \theta Z$ and therefore $\theta L$ has a fourth point $\langle\theta L-\{a\}\rangle$. This is impossible since $M$ is binary. (5.35).

We may now suppose that $X, Y$, and $Z$ are on the same plane $P$ of $\boldsymbol{M}^{\prime}$, but not on a common line. $P$ is connected, by 6.24 Let $X_{1}, Y_{1}$, and $Z_{1}$ be the third points on the connected lines $Y \cup Z, Z \cup X$ and $X \cup Y$ of $M^{\prime}$ respectively

Suppose first that two of the points $X_{1}, Y_{1}$, and $Z_{1}$ are on a common line $L$ of $M^{\prime}$. There is a cell $d$ of $P$ such that $L=\langle P-\{d\}\rangle$, by 7.11. It is clear that $L$ is not on $X, Y$, or $Z$. Hence $d \epsilon X \cap Y \cap Z$. We thus have

$$
u(Q)=s(X, d) s(Y, d) s(Y, d) s(Z, d) s(Z, d) s(X, d)=1,
$$

which is contrary to the choice of $Q$.

We conclude that no two of $X_{1}, Y_{1}$, and $Z_{1}$ are on a common line of $M^{\prime}$. Any line through one of them is therefore on $X, Y$, or $Z$, by 5.35 . Hence, by 4.26 , there are six distinct lines on $P$, each on one of the points $X, Y$, and $Z$.

These six lines are mapped by $\theta$ onto six distinct lines of $M$ on the plane $\theta P$, each being on one of the points $\theta X, \theta Y$, and $\theta Z$ and therefore having $a$ as a cell. But then there is a seventh line $\langle\theta P-\{a\}\rangle$ on $\theta P$, by 7.11. Accordingly $M$ includes a Fano configuration. Case III. $Q$ is of the third kind.

We now have $Q=(X, Y, Z, T, X)$, where $X, Y, Z$, and $T$ are distinct points on a plane $P$ of $M^{\prime}$ such that no three are on a common line. We can therefore find cells $b \epsilon P-(X \cup Y), \quad c \epsilon P-(Y \cup Z), \quad d \epsilon P-(Z \cup T)$, and $e \epsilon P-(T \cup X)$. Then $b$ belongs to $Z$ and $T$ but not to $X$ or $Y, c$ belongs to $T$ and $X$ but not to $Y$ or $Z$, and so on.

If the chains $g(X), g(Y), g(Z)$, and $g(T)$ are linearly independent we can construct a nonzero linear combination of them, $h$ say, such that $h(b)=h(c)=h(d)$ $=0$. Then there is a point $U$ of $M^{\prime}$ contained in $\|h\|$, by 5.43 . This point is common to the lines $\langle P-\{b\}\rangle$ $=X \cup Y, \quad\langle P-\{c\}\rangle=Y \cup Z$ and $\langle P-\{d\}\rangle=Z \cup T$. Hence $U=Y=Z$. But this is impossible since $Y$ and $Z$ are distinct. We deduce that $g(X), g(Y), g(Z)$, and $g(T)$ are in fact linearly dependent. We thus have 


$\left|\begin{array}{cccc}0 & 0 & s(T, c) & s(T, b) \\ s(Z, e) & 0 & 0 & s(Z, b) \\ s(Y, e) & s(Y, d) & 0 & 0 \\ 0 & s(X, d) & s(X, c) & 0\end{array}\right|=0$,

that is

$s(Z, b) s(T, c) s(X, d) s(Y, e)$

$$
-s(T, b) s(X, c) s(Y, d) s(Z, e)=0
$$

It follows from this result that

$$
u(Q)=t(X, Y) t(Y, Z) t(Z, T) t(T, X)=1 .
$$

Case IV. $Q$ is of the fourth kind.

In this case there is a box on some 3-flat $J$ of $M^{\prime}$ such that the six sides $P_{1}, P_{2}, \ldots ., P_{6}$ of the box have the following properties, Each is on just two points of $C$ and no two of the $P_{i}$ have more than one point of $C$ in common. By the definition of a box no three of the planes $P_{i}$ are on a common line.

It follows that no three of the six planes $\theta P_{i}$ of $M$ on $\theta J$ have a common line, and that each of them has $a$ as a cell. Moreover each line of intersection of two planes $\theta P_{i}$ is of the form $\theta L$, where $L$ is the line of intersection of the corresponding planes $P_{i}$ in $M^{\prime}$. $L$ is on some point of $M^{\prime}$ not in $C$ and therefore $\theta L$ has $a$ as a cell.

The plane $\langle\theta J-\{a\}\rangle$ of $M$ on $\theta J$ is thus distinct from each of the six planes $\theta P_{i}$ and is not on any of their lines of intersection. Hence $M$ includes a heptahedron.

In each of the four cases we have deduced a contradiction, either of the hypothesis of 7.41 or of our assumption that 7.414 is false. We deduce that 7.414 is in fact valid.

We can partition the set of points of $M^{\prime}$ not in $C$ into disjoint non-null classes $K_{1}, K_{2}, \ldots, K_{q}$ such that for any two points $X$ and $Y$ of $M^{\prime}$ off $C$ there is a path from $X$ to $Y$ off $C$ in $M^{\prime}$ if and only if $X$ and $Y$ belong to the same class $K_{j}$. For each class $K_{j}$ we select an arbitrary representative $T_{j}$ and write

$$
v\left(T_{j}\right)=1 .
$$

For any other point $X$ in $K_{j}$ we write

$$
v(X)=u(Q) v\left(T_{i}\right),
$$

where $Q$ is any path from $T_{i}$ to $X$ off $C$ in $M^{\prime}$.

This definition determines the integer $v(X)$ uniquely. For suppose $Q^{\prime}$ is another path from $T_{i}$ to $X$ off $C$ in $M^{\prime}$. Then

$$
\begin{gathered}
u(Q)=u\left(Q^{-1}\right)=u\left(Q^{-1}\right) u\left(Q^{\prime}\right) u\left(Q^{\prime}\right) . \\
=u\left(Q^{-1} Q^{\prime}\right) u\left(Q^{\prime}\right) \\
=u\left(Q^{\prime}\right),
\end{gathered}
$$

by 7.414 since $Q^{-1} Q^{\prime}$ is re-entrant.
For each $U \epsilon M$ we define a chain $f_{U}$ over the ring of integers as follows.

Suppose first that $a \epsilon U$. If $U=\{a\}$ we write $f_{U}(a)$ $=1$, and $f_{U}(b)=0$ whenever $b \neq a$. If $U \neq\{a\}$, then $U-\{a\}$ is a non-null flat of $M^{\prime}$, by 4.45. The flat $\theta(U-\{a\})$ of $M$ is $U-\{a\}$ or $U$, and the former alternative is ruled out by Axiom I. Hence $U-\{a\}$ is a point $X$ of $M^{\prime}$ not in $C$. We choose $f_{U}$ to agree with $g(X)$ in $E-\{a\}$, and to satisfy

$$
f_{U}(A)=v(X) .
$$

Suppose next that $a \notin U$. Then $U$ is a flat of $M^{\prime}$, by 4.45 , and $\theta U$ is $U$ or $U \cup\{a\}$.

If $\theta U=U$, then $U$ is a point of $M^{\prime}$. We then choose $f_{U}$ to agree with $g(U)$ in $E-\{a\}$ and to satisfy

$$
f_{U}(a)=0
$$

If instead $\theta U=U \cup\{a\}$ then $U \cup\{a\}$ is a line of $M$, by 4.13 , and $U$ is a line of $M^{\prime}$, by 4.44 . Now each point on $U$ in $M^{\prime}$ is mapped by $\theta$ onto a point of $M$ on $U \cup\{a\}$. But there are at most three points of $M$ on $U \cup\{a\}$, by 5.35 , and one of these is $\mathrm{U}$. Hence $\mathrm{U}$ must be a disconnected line of $\boldsymbol{M}^{\prime}$, having only two points $X$ and $Y$. Write $V=\theta X$ and $W=\theta Y$. Then $V=X \cup\{a\}$ and $W=Y \cup\{a\}$, by Axiom I. Thus $f_{\mathrm{V}}$ and $f_{W}$ are already defined. We take either $f_{U}$ or $-f_{U}$ to be

$$
f_{\mathrm{V}}(a) f_{W}-f_{W}(a) f_{\mathrm{V}} .
$$

We note that for each atom $U$ of $M$ we have $\left\|f_{U}\right\|=U$ and that the coefficients in each $f_{U}$ are restricted to the values $1,-1$ and 0 . Moreover the restriction of $f_{U}$ to $E-\{a\}$ is a chain of $N^{\prime}$.

7.415 Let $U, V$, and $W$ be the three points on a connected line $L$ of $M$. Then $f_{U}, f_{V}$ and $f_{W}$ are linearly dependent.

Proof: We discuss first the case $a \epsilon L$.

We may suppose $a \epsilon V \cup W$ and $a \notin U$, by 7.12. Then there are points $X=V-\{a\}$ and $Y=W-\{a\}$ of $M^{\prime}$ such that $\theta X=V$ and $\theta Y=W$. Write $L^{\prime}=L \cap(E-\{a\})$ $=L-\{a\}$. Then $L^{\prime}$ is a flat of $M^{\prime}$, by 4.45 . It clearly satisfies $\theta L^{\prime}=L$ and is therefore a line of $M^{\prime}$. The set $U$ is also a flat of $M^{\prime}$, by 4.45 . It is either the line $L^{\prime}$ or a point of $M^{\prime}$ on it.

If $U$ is a point of $M^{\prime}$ on $L^{\prime}$, then $\theta U=U$, and $U$ is distinct from $X$ and $Y$. The line $L^{\prime}$ is thus connected, by 4.23 . We can define $v(X)$ by a path $Q$ off $C$ from a representative point $T_{i}$ to $X$, and then define $v(Y)$ by the path $Q(X, Y)$. We then have

$$
v(X) v(Y)=u(Q) v\left(T_{i}\right) \cdot u(Q) t(X, Y) v\left(T_{i}\right)=t(X, Y) .
$$

Hence for each $b \epsilon X \cap Y$ we have $v(X) v(Y)=s(X, b) s(Y, b)$. By 7.12 this implies

$$
\|v(X) g(Y)-v(Y) g(X)\| \subseteq U .
$$

since $M^{\prime}$ is binary. But the expression on the left is non-null by Axiom I, and since $g(U)$ is an elementary 
chain of $N^{\prime}$ it follows that

$$
g(U)= \pm(v(X) g(Y)-v(Y) g(X)) .
$$

Hence, by the definitions of $f_{U}, f_{V}$, and $f_{W}$, we have

$$
f_{U}= \pm\left(f_{V}(a) f_{W}-f_{W}(a) f_{V}\right) .
$$

If instead $U$ is the line $L^{\prime}$ of $M^{\prime}$ we have $\theta U=U \cup\{a\}$ $=L$. Then the preceding equation follows at once from the definition of $f_{U}$. The required linear dependence is thus established for the case $a \epsilon L$.

Now suppose $a \notin L$. It may happen that $L$ is a line of $M^{\prime}$ so that $\theta L=L$. Then $U, V$, and $W$ are flats of $M^{\prime}$, and therefore points of $\boldsymbol{M}^{\prime}$ on $L$, by 4.14. There is a linear relation between $g(U), g(V)$, and $g(W)$, as we may see by applying 2.31 to the regular chaingroup $N^{\prime} \times L$. A corresponding relation holds between $f_{U}, f_{V}$, and $f_{W}$.

In the remaining case $L$ is a flat, but not a line, of $M^{\prime}$, by 4.45. Then $\theta L=L \cup\{a\}$, and $\theta L$ is a plane of $M$. It is a connected plane since some point $X$ of $M^{\prime}$ on $L$ must satisfy $a \epsilon \theta X$. (See sec 4.4.) By 4.26 there are connected lines $L_{U}, L_{V}$ and $L_{W}$ on $\theta L$, distinct from $L$, which are on $U, V$, and $W$ respectively.

We can choose these lines to have no common point. For suppose $T$ is such a common point. There is a point $T^{\prime}$, distinct from $T$ and $U$, on $L_{U}$. By 4.26 there is a connected line $L^{\prime}$, distinct from $L_{U}$, on $\theta L$ and $T^{\prime}$. Without loss of generality we may suppose $L^{\prime}$ to meet $L$ in $V$, by 5.35. Replacing $L_{V}$ by $L^{\prime}$ we obtain three lines of the kind required.

Write $\quad X=\left\langle L_{V} \cap L_{W}\right\rangle, \quad Y=\left\langle L_{W} \cap L_{U}\right\rangle \quad$ and $\quad Z$ $=\left\langle L_{U} \cap L_{V}\right\rangle$. We note that $\langle\theta L-\{a\}\rangle=L$, by 7.11. Hence $a \in X \cap Y \cap Z$. We may therefore apply the result already proved to $L_{U}, L_{V}$, and $L_{W}$, and obtain

$$
\begin{gathered}
f_{U}= \pm\left(f_{Y}(a) f_{Z}-f_{Z}(a) f_{Y}\right), \\
f_{V}= \pm\left(f_{Z}(a) f_{X}-f_{X}(a) f_{Z}\right), \\
f_{W}= \pm\left(f_{X}(a) f_{Y}-f_{Y}(a) f_{X}\right) .
\end{gathered}
$$

Thus $f_{U}, f_{V}$, and $f_{W}$ are linearly dependent in this case also. This completes the proof of 7.415.

The preceding result shows that the chains $f_{U}$ corresponding to the atoms of $M$ satisfy the conditions of 5.11. They therefore generate an integral chaingroup $N$ such that $M=M(N)$. But the coefficients in each $f_{U}$ are restricted to the values $1,-1$ and 0 . Hence the $f_{U}$ are primitive chains of $N$. It follows that $N$ is a regular chain-group and $M$ is a regular matroid. We have thus completed the proof of 7.41.

\subsection{Characterization of Regular Matroids}

Combining the results $5.53,7.21,7.31$, and 7.41 we have the theorem.

7.51 A binary matroid $M$ is regular if and only if it has no minor of Type BI or BII.

An equivalent condition is that $M$ shall include no Fano configuration or heptahedron.
We may also apply 5.26 and 5.23 to obtain the following rule.

7.52 A binary matroid $M$ is non-regular if and only if some standard representative matrix $K$ of $M$ has a submatrix $J$ such that either $J$ or its transpose is of the following form, to within a permutation of columns.

$$
\left\{\begin{array}{llll}
1 & 0 & 1 & 1 \\
1 & 1 & 0 & 1 \\
0 & 1 & 1 & 1
\end{array}\right\}
$$

In one respect the preceding theory is incomplete: we still need a convenient algorithm for determining whether a given binary matroid is regular.

\section{The Matroid and the Atom}

\subsection{General Matroid}

In this chapter we suppose given a matroid $M$ on a set $E$, and we fix some atom $Y$ of $M$. We discuss theorems concerned with the relationship of $Y$ to the rest of the matroid.

We refer to the elementary separators of $M \cdot(E-Y)$ as the bridges of $Y$ in $M$. Such a bridge is trivial if it contains no atom of $M \cdot(E-Y)$, and monatomic if it contains just one. A trivial bridge of $Y$ consists of a single cell of $E-Y$ contained in no atom of $M \cdot(E-Y)$, and every such cell of $E-Y$ defines a trivial bridge of $Y$. A nontrivial bridge of $Y$ in $M$ is thus a flat of $M \cdot(E-Y)$.

$8.11 d(M \cdot(E-Y))=d M-1$.

Proof: Since $d(M \times Y)=d Y=0$ this follows from 3.54

8.12 Suppose $S \subset E-Y$ and let $d$ be an integer $\geqslant-1$. Then $S$ is $a$ d-flat of $M \cdot(E-Y)$ if and only if $S \cup Y$ is $a(d+1)$-flat of $M$.

Proof: Suppose $S$ is a flat of $M \cdot(E-Y)$. Then each atom of $M \cdot(E-Y)$ on $S$ is the intersection with $E-Y$ of an atom of $M$ contained in $S \cup Y$. Hence $S \cup Y$ is a union of atoms of $M$, that is a flat of $M$.

Conversely suppose $S \cup Y$ is a flat of $M$. Each cell of $S$ belongs to an atom of $M$ on $S \cup Y$ and therefore to an atom of $M \cdot(E-Y)$ on $S$, by 1.11 and the definition of a reduction. Hence $S$ is a flat of $M \cdot(E-Y)$.

To complete the proof we observe that

$$
d((M \cdot(E-Y)) \times S)=d((M \times(S \cup Y)) \cdot S)
$$

$$
=d(M \times(\mathrm{S} \cup Y))-1,
$$

by 3.333 and 8.11 .

8.13 Let $S$ be a connected flat of $M \cdot(E-Y)$. Then the only possible non-trivial separators of $M \times(S \cup Y)$ are $S$ and $Y$. Moreover $S$ and $Y$ are separators of $M \times(S \cup Y)$ if and only if

$$
(M \cdot(E-Y)) \times S=M \times S .
$$


Proof: Let $Z$ be a separator of $M \times(S \cup Y)$. Then either $Y \subseteq Z$ or $Y \cap Z=\phi$. Moreover $Z \cap S$ is a separator of $(M \times(S \cup Y)) \cdot S$, by 3.43 , and this matroid is $(M \cdot(E-Y)) \times S$, by 3.334 . Hence $S \subseteq Z$ or $S \cap Z=\phi$, by hypothesis. Accordingly $Y$ and $S$ are the only possible nontrivial separators of $M \times(S \cup Y)$.

The necessary and sufficient condition for $S$ and $Y$ to be separators of $M \times(S \cup Y)$ is

$$
(M \times(S \cup Y)) \cdot S=(M \times(S \cup Y)) \times S,
$$

by 3.42 . This is equivalent to the condition stated in the enunciation, by 3.331 and 3.333 .

In what follows we shall be concerned only with binary matroids, and we do not pursue the general theory any further.

\subsection{Binary Matroid}

From now on we suppose $M$ binary. If $Z \epsilon M \cdot(E-Y)$ then $Y \cup Z$ is a line of $M$, by 8.12 . If it is connected it has just two points other than $Z$, by 5.35 . Their intersections with $Y$ are complementary non-null subsets $T$ and $U$ of $Y$, by 7.12. We call these subsets the primary segments of $Y$ determined by $Z$. The unordered pair $\{T, U\}$ is the partition of $Y$ determined by $Z$. If $Y \cup Z$ is not connected it has only two points $Y$ and $Z$. In this case it is convenient to say that the partition of $Y$ determined by $Z$ is $\{Y, \phi\}$, and that the corresponding primary segments are $Y$ and $\phi$.

Let $B$ be any bridge of $Y$ in $M$. If there is an atom of $M \cdot(E-Y)$ on $B$ we make the following definition. A segment of $Y$ determined by $B$, briefly a " $B$-segment" of $Y$ is a minimal non-null intersection of primary segments of $Y$ determined by atoms of $M \cdot(E-Y)$ on $B$. If $B$ is trivial we say that the only $B$-segment of $Y$ is $Y$ itself. Evidently we have

8.21 The B-segments of $Y$ are disjoint non-null subsets of $Y$ whose union is $Y$.

The class of $B$-segments of $Y$ is the partition of $Y$ determined by $B$. We denote it by $\pi(M, B, Y)$.

Two bridges $B_{1}$ and $B_{2}$ of $Y$ in $M$ will be said to avoid one another if there exists $S_{1} \epsilon \pi\left(M, B_{1}, Y\right)$ and $S_{2} \epsilon \pi\left(M, B_{2}, Y\right)$ such that $S_{1} \cup S_{2}=Y$, and to overlap one another in the contrary case.

The atom $Y$ is bridge-separable in $M$ if its bridges can be classified in two disjoint classes $P$ and $Q$ so that no two bridges belonging to the same class overlap. In an extreme case $Y$ may have no two bridges which overlap. We then say $Y$ is totally bridge-separable in $M$.

We call a binary matroid $M$ an even matroid if every atom is bridge-separable.

The following examples may clarify our terminology.

Consider a binary matroid of Type BI. This is the matroid of a binary chain-group $N$ which, with an appropriate enumeration of cells, has the following standard representative matrix.

$$
K=\left\{\begin{array}{lllllll}
1 & 0 & 0 & 1 & 0 & 1 & 1 \\
0 & 1 & 0 & 1 & 1 & 0 & 1 \\
0 & 0 & 1 & 0 & 1 & 1 & 1
\end{array}\right\}
$$
by $a_{j}$.

Let us denote the cell corresponding to the $j^{\text {th }}$ column

We take $Y$ to be the atom $\left\{a_{3}, a_{5}, a_{6}, a_{7}\right\}$ corresponding to the third row of $K$. Then $E-Y=\left\{a_{1}, a_{2}, a_{4}\right\}$. (See 5.22.)

It is clear that the only nonzero chains of $N \cdot(E-Y)$ are those with representative vectors $(1,0,1),(0,1,1)$ and their sum $(1,1,0)$. Moreover these three chains are elementary. Accordingly the matroid $M \cdot(E-Y)$ $=M(N \cdot(E-Y))$ has just three atoms $\left\{a_{1}, a_{2}\right\},\left\{a_{2}, a_{4}\right\}$ and $\left\{a_{1}, a_{4}\right\}$. (See 3.31.)

We observe that $M \cdot(E-Y)$ is connected. Hence $\left\{a_{1}, a_{2}, a_{4}\right\}$ is the only bridge of $Y$ in $M$. Accordingly $Y$ must be classed as bridge-separable.

It can be verified that all seven atoms of $M$ are bridge-separable. Indeed they are all equivalent under the symmetry of the Fano configuration. Hence the matroids of Type BI are even.

In our example the sets $\left\{a_{1}, a_{4}\right\} \cup Y$ and $\left\{a_{2}, a_{4}\right\} \cup Y$ are lines of $M$, by 8.12. They are on points corresponding to the first and second rows of $K$ respectively. We deduce that they determine the partitions $\left\{\left\{a_{3}, a_{5}\right\}\right.$, $\left.\left\{a_{6}, a_{7}\right\}\right\}$ and $\left\{\left\{a_{3}, a_{6}\right\},\left\{a_{5}, a_{7}\right\}\right\}$ of $Y$ respectively.

Considering the intersections of these four primary segments we see that

$$
\pi(M, B, Y)=\left\{\left\{a_{3}\right\},\left\{a_{5}\right\},\left\{a_{6}\right\},\left\{a_{7}\right\}\right\} .
$$

Next we discuss binary matroids of Type BII. Such a matroid $M$ corresponds to a binary chain-group $N$ with the following representative matrix $K$, by 5.23 .

$$
K=\left\{\begin{array}{lllllll}
1 & 0 & 0 & 0 & 1 & 1 & 0 \\
0 & 1 & 0 & 0 & 0 & 1 & 1 \\
0 & 0 & 1 & 0 & 1 & 0 & 1 \\
0 & 0 & 0 & 1 & 1 & 1 & 1
\end{array}\right\}
$$

Again we enumerate the cells as $a_{1}, a_{2}, \ldots ., a_{7}$, in the order of the columns of $K$.

We take $Y$ to be the atom $\left\{a_{4}, a_{5}, a_{6}, a_{7}\right\}$ corresponding to the last row of $K$. Now each of the sets $\left\{a_{1}\right\}$, $\left\{a_{2}\right\}$ and $\left\{a_{3}\right\}$ are domains of chains of $N \cdot(E-Y)$. Hence these three sets are the only atoms of $M \cdot(E-Y)$, by Axiom I. Accordingly they are also the elementary separators of $M \cdot(E-Y)$, that is they are the three bridges of $Y$ in $M$.

The line $Y \cup\left\{a_{1}\right\}$ of $M$ is on a point corresponding to the first row of $M$. We deduce that the atom $\left\{a_{1}\right\}$ of $M \cdot(E-Y)$ determines the partition $\left\{\left\{a_{4}, a_{7}\right\}\right.$, $\left.\left\{a_{5}, a_{6}\right\}\right\}$ of $Y$. Since $\left\{a_{1}\right\}$ is a monatomic bridge of 
$Y$ in $M$ we thus have

$$
\pi\left(M,\left\{a_{1}\right\}, Y\right)=\left\{\left\{a_{4}, a_{7}\right\},\left\{a_{5}, a_{6}\right\}\right\} .
$$

Similarly

$$
\begin{aligned}
& \pi\left(M,\left\{a_{2}\right\}, Y\right)=\left\{\left\{a_{4}, a_{5}\right\},\left\{a_{6}, a_{7}\right\}\right\}, \\
& \pi\left(M,\left\{a_{3}\right\}, Y\right)=\left\{\left\{a_{4}, a_{6}\right\},\left\{a_{5}, a_{7}\right\}\right\} .
\end{aligned}
$$

Inspection of these formulae shows that the three bridges of $Y$ in $M$ are mutually overlapping. Hence $Y$ is not bridge-separable and the binary matroid $M$ is odd, that is not even.

8.22 Every binary matroid of Type BII is odd.

\subsection{Kuratowski Graphs}

We now show the application of our terminology to certain matroids associated with graphs.

Suppose first that $G$ is the complete 5 -graph shown in figure $5 \mathrm{~B}$. We use the notation for it given at the end of chapter 5 .

Let $M$ be the polygon-matroid of $G$, so that atoms of $M$ correspond to polygons of $G$. In particular there is an atom

$$
Y=\left\{L_{12}, L_{23}, L_{34}, L_{45}, L_{15}\right\}
$$

of $M$ corresponding to the outer polygon in figure 5B.

Now $M \cdot(E-Y)$ is the polygon matroid of $G \times(E-Y)$, by 3.372 . In this graph the polygon $G \cdot Y$ has been replaced by a single vertex $v$, and each cell of $E-Y$ has become a loop whose ends coincide in $v$. Accordingly each cell of $E-Y$ constitutes by itself an atom of $M \cdot(E-Y)$. It also constitutes an elementary separator of $M \cdot(E-Y)$, by Axiom I. We deduce that there are just five bridges of $Y$ in $M$, namely the sets

$$
\left\{L_{13}\right\},\left\{L_{24}\right\},\left\{L_{35}\right\},\left\{L_{14}\right\},\left\{L_{25}\right\} \cdot
$$

Considering the triangle defined by $L_{12}, L_{23}$, and $L_{13}$ we see that $\left\{L_{13}\right\}$ determines the partition $\left\{\left\{L_{12}\right.\right.$, $\left.\left.L_{23}\right\},\left\{L_{34}, L_{45}, L_{15}\right\}\right\}$ of $Y$. Hence, using the symmetry of $G$, we have

$$
\begin{aligned}
& \pi\left(M,\left\{L_{13}\right\}, Y\right)=\left\{\left\{L_{12}, L_{23}\right\},\left\{L_{34}, L_{45}, L_{15}\right\}\right\}, \\
& \pi\left(M,\left\{L_{24}\right\}, Y\right)=\left\{\left\{L_{23}, L_{34}\right\},\left\{L_{45}, L_{15}, L_{12}\right\}\right\},
\end{aligned}
$$

and so on.

Inspection of these formulae shows that $\left\{L_{13}\right\}$ overlaps $\left\{L_{24}\right\},\left\{L_{24}\right\}$ overlaps $\left\{L_{35}\right\},\left\{L_{35}\right\}$ overlaps $\left\{L_{14}\right\},\left\{L_{14}\right\}$ overlaps $\left\{L_{25}\right\}$ and $\left\{L_{25}\right\}$ overlaps $\left\{L_{13}\right\}$. It is thus impossible to arrange these five bridges in two classes so that no two members of the same class overlap. Thus $Y$ is not bridge-separable.

Next let $G$ be a Thomsen graph as shown in the second diagram of figure 5B. A similar analysis applies.

We write $M=P(G)$ and take $Y=\left\{L_{11}, L_{21}, L_{22}, L_{32}\right.$, $\left.L_{33}, L_{13}\right\}$, corresponding to the outer polygon of the diagram. Then we find that $\left\{L_{12}\right\},\left\{L_{23}\right\}$, and $\left\{L_{31}\right\}$ are the three atoms of $M \cdot(E-Y)$ and the three bridges of $Y$ in $M$. Considering the quadrilaterals such as $a_{1} b_{1} a_{2} b_{2}$ we arrive at the following partition formulae.

$$
\begin{aligned}
& \pi\left(M,\left\{L_{12}\right\}, Y\right)=\left\{\left\{L_{11}, L_{21}, L_{22}\right\},\left\{L_{32}, L_{33}, L_{13}\right\}\right\}, \\
& \pi\left(M,\left\{L_{23}\right\}, Y\right)=\left\{\left\{L_{22}, L_{32}, L_{33}\right\},\left\{L_{13}, L_{11}, L_{21}\right\}\right\}, \\
& \pi\left(M,\left\{L_{31}\right\}, Y\right)=\left\{\left\{L_{33}, L_{13}, L_{11}\right\},\left\{L_{21}, L_{22}, L_{32}\right\}\right\} .
\end{aligned}
$$

We see from these formulae that the three bridges are mutually overlapping. Hence $Y$ is not bridgeseparable.

We say that a matroid is of Type KI or KII if it can be interpreted as the polygon-matroid of a complete 5 -graph or a Thomsen graph respectively. We sum up the results of the present section in the following theorem

\subsection{Every matroid of Type KI or KII is odd.}

\subsection{Skewness}

In this section $M$ denotes an arbitrary binary matroid and $Y$ denotes an atom of $M$.

We say that an atom $Z$ of $M \cdot(E-Y)$ cuts a subset $S$ of $Y$ if it determines a partition $\{T, U\}$ of $Y$ such that $S \cap T$ and $S \cap U$ are both non-null.

8.41 The points of $M \cdot(E-Y)$ which do not cut a given subset $S$ of $Y$ constitute a linear subclass of $M \cdot(E-Y)$.

Proof: Let $Z_{1}, Z_{2}$, and $Z_{3}$ be the three points on some connected line $L$ of $M \cdot(E-Y)$. Suppose $Z_{1}$ and $Z_{2}$ do not cut $S$. Then each point on the lines $Y \cup Z_{1}$ and $Y \cup Z_{2}$ of $M$ either contains $S$ or does not meet $S$. That is it does not "cut" $S$ in the sense of section 4.3 .

Let $X_{3}$ be any point of $M$, other than $Y$, on the line $Y \cup Z_{3}$. There is a line $L^{\prime}$ of $M$ on $X_{3}$ and the plane $Y \cup L$ which is not on $Y$, by 4.15. This line meets $Y \cup Z_{1}$ and $Y \cup Z_{2}$ in two distinct points of $M$, by 4.171. Hence $X_{3}$ either contains $S$ or does not meet $S$, by 4.31. It follows that the point $Z_{3}$ of $M \cdot(E-Y)$ does not cut $S$.

We say that two points $Z$ and $Z^{\prime}$ of $M \cdot(E-Y)$ are skew with respect to $Y$ if they determine partitions $\{T, U\}$ and $\left\{T^{\prime}, U^{\prime}\right\}$ of $Y$ such that the intersections $T \cap T^{\prime}, T \cap U^{\prime}, U \cap T^{\prime}$ and $U \cap U^{\prime}$ are all non-null.

We extend this definition by saying that two bridges $B$ and $B^{\prime}$ of $Y$ in $M$ are skew if there are points $Z$ and $Z^{\prime}$ of $M \cdot(E-Y)$, skew with respect to $Y$, which are on $B$ and $B^{\prime}$ respectively. We also say that a point $Z$ of $M \cdot(E-Y)$ is skew to a bridge $B$ of $Y$ in $M$ if there is a point $Z^{\prime}$ of $M \cdot(E-Y)$ on $B$ which is skew to $Z$.

8.42 Let $B_{1}, B_{2}$ and $B_{3}$ be distinct bridges of $Y$ in $M$ such that $B_{2}$ is skew both to $B_{1}$ and to $B_{3}$. Then either there is a point $Z_{2}$ of $M \cdot(E-Y)$ on $B_{2}$ which is skew to both $B_{1}$ and $B_{3}$ or there are points $Z_{1}$ and $Z_{3}$ of $M \cdot(E-Y)$ on $B_{1}$ and $B_{3}$ respectively such that $Z_{1}, B_{2}$, and $Z_{3}$ are mutually skew.

Proof: There are points $Z_{1}, Z_{2}^{\prime}, Z_{2}^{\prime \prime}$, and $Z_{3}$ of $M \cdot(E-Y)$, on $B_{1}, B_{2}, B_{2}$, and $B_{3}$ respectively, such that $Z_{1}$ is skew to $Z_{2}^{\prime}$ and $Z_{2}^{\prime \prime}$ is skew to $Z_{3}$. If $Z_{1}$ and 
$Z_{3}$ are skew the second alternative of the theorem holds.

In the remaining case $Z_{1}$ and $Z_{3}$ determine partitions $\left\{S_{1}, T_{1}\right\}$ and $\left\{S_{3}, T_{3}\right\}$ of $Y$ such that $T_{1} \cap T_{3}=\phi$, that is $T_{1} \subseteq S_{3}$ and $T_{3} \subseteq S_{1}$.

Now $Z_{2}^{\prime}$ cuts $T_{1}, \bar{Z}_{2}^{\prime \prime}$ cuts $T_{3}$, and $B_{2}$ is a connected flat of $M \cdot(E-Y)$. Hence, by 4.37 and 8.41 there is a point $Z_{2}$ of $M \cdot(E-Y)$ on $B_{2}$ cutting both $T_{1}$ and $T_{3}$. But then $Z_{2}$ is skew te both $Z_{1}$ and $Z_{3}$, that is to both $B_{1}$ and $B_{3}$.

Let $B$ be any bridge of $Y$ in $M$. We call it an $n$-bridge of $Y$, where $n$ is an integer, if

$$
|\pi(M, B, Y)|=n .
$$

Two $n$-bridges $B_{1}$ and $B_{2}$ of $Y$ in $M$ are equipartite if

$$
\pi\left(M, B_{1}, Y\right)=\pi\left(M, B_{2}, Y\right)
$$

8.43 Let $B$ be a 2-bridge or 3-bridge of $Y$ in $M$, and suppose $W \epsilon \pi(M, B, Y)$. Then there is a point $Z$ of $M \cdot(E-Y)$ on $B$ which determines the partition $\{W, Y-W\}$ of $Y$.

Proof: If $B$ is a 2-bridge this result follows at once from the definitions. We may therefore write $\pi(M$, $B, Y)=\{U, V, W\}$. There is a point of $M \cdot(E-Y)$ on $B$ which cuts $U \cup W$ and another which cuts $V \cup W$. Hence, by 4.37 and 8.41 there is such a point $Z$ which cuts both. This can only determine the partition $\{W, U \cup V\}$ of $Y$.

The next theorem shows the relation between skewness and overlapping for bridges.

8.44 Let $B_{1}$ and $B_{2}$ be overlapping bridges of $Y$ in $M$. Then either $B_{1}$ and $B_{2}$ are skew or they are equipartite 3-bridges.

Proof: Assume $B_{1}$ and $B_{2}$ are not skew.

Let $Z_{1}$ be a point of $M \cdot(E-Y)$ on $B_{1}$ determining a partition $\{S, T\}$ of $Y$. Suppose $S$ and $T$ each meet more than one member of $\pi\left(M, B_{2}, Y\right)$. Then $S$ and $T$ are each cut by some point of $M \cdot(E-Y)$ on $B_{2}$, and therefore there is one such point which cuts both of them, by 4.37 and 8.41. But then $B_{1}$ and $B_{2}$ are skew, contrary to assumption. We deduce that one of $S$ and $T$ must be contained in a member of $\pi\left(M, B_{2}, Y\right)$.

Suppose $B_{1}$ is a 2-bridge or 3-bridge. Then if $W \epsilon \pi\left(M, B_{1}, Y\right)$ we can apply the preceding result with $W=S$ and $Y-W=T$, by 8.43 . We find that there exists $U \epsilon \pi\left(M, B_{2}, Y\right)$ such that either $W \subseteq U$ or $Y-W \subseteq U$. The second alternative must be ruled out since it implies $W \cup U=Y$, contrary to the hypothesis that $B_{1}$ and $B_{2}$ overlap. We deduce further that $B_{1}$ is not a 2-bridge since then $Y-W \epsilon \pi\left(M, B_{1}, Y\right)$ and both alternatives are ruled out.

Suppose $B_{1}$ is a 3 -bridge. Write $\pi\left(M, B_{1}, Y\right)$ $=\{U, V, W\}$. Then by the above reasoning there are members $U^{\prime}, V^{\prime}$, and $W^{\prime}$ of $\pi\left(M, B_{2}, Y\right)$ such that $U \subseteq U^{\prime}, V \subseteq V^{\prime}$, and $W \subseteq W^{\prime}$. These three $B_{2}$-segments are distinct. If for example we had $U^{\prime}=V^{\prime}$ it would follow that $U^{\prime} \cup W=Y$, contrary to hypothesis. It follows that $B_{1}$ and $B_{2}$ are equipartite 3-bridges.

We have still to consider the case in which $B_{1}$ is an $n$-bridge, with $n \geqslant 4$.
Let $W_{1}, W_{2}, W_{3}$, and $W_{4}$ be distinct members of $\pi\left(M, B_{1}, Y\right)$. Using 4.37 and 8.41 we find that there is a point $Z$ of $M \cdot(E-Y)$ on $B_{1}$ which cuts both $W_{1} \cup W_{2}$ and $W_{3} \cup W_{4}$. It determines a partition $\{S, T\}$ of $Y$ such that $W_{1}$ and $W_{2}$ are in different sets $S$ and $T$, as are also $W_{3}$ and $W_{4}$.

By the first part of the proof we can find $U^{\prime} \epsilon \pi(M$, $\left.B_{2}, Y\right)$ such that $U^{\prime}$ contains two of the given four $B_{1-}$ segments. Let $W^{\prime}$ be a member of $\pi\left(M, B_{2}, Y\right)$ containing the greatest possible number $k$ of $B_{1}$-segments. $(k \geqslant 2)$.

Suppose there are at least two $B_{1}$-segments not in $W^{\prime}$. Then by the result just proved for four $B_{1}$-segments $W_{1}, W_{2}, W_{3}, W_{4}$ it follows that some point of $M \cdot(E-Y)$ on $B_{1}$ determines a partition $\{S, T\}$ of $Y$ such that $S$ and $T$ each meet both $W^{\prime}$ and $Y-W^{\prime}$. Since some member of $\pi\left(M, B_{2}, Y\right)$ must contain $S$ or $T$ we have a contradiction. We are forced to conclude that there is at most one $B_{1}$-segment which is not contained in $W^{\prime}$. But then $B_{1}$ and $B_{2}$ avoid one another, contrary to hypothesis.

This completes the proof of the theorem.

\subsection{Segments Determined by Bridges}

This section deals with some auxiliary results to be used in later proofs. They enable us to go from $Y$ to another atom of the binary matroid $M$, or to replace $M$ by one of its minors.

8.51 Let $S$ be a subset of $E$, and let $X$ be an atom of $M$ such that $X \cap S$ is non-null. Then $X \cap S$ is a union of disjoint atoms of $M \cdot S$.

Proof: Let $N$ be the binary chain-group such that $M=M(N)$. Then $M \cdot S=M(N \cdot S)$, by 3.31. But $X \cap S$ is the domain of a chain of $N \cdot S$. The theorem now follows from 5.32 .

8.52 Let $B$ be a bridge of $Y$ in $M$. Then the B-segments of $Y$ in $M$ are the minimal non-null intersections of atoms of $(M \times(B \cup Y)) \cdot Y$.

Proof. Let the class of minimal non-null intersections of atoms of $(M \times(B \cup Y)) \cdot Y$ be denoted by $J$. The members of $J$ are disjoint and their union is $Y$, by 1.11 .

Let $a$ be any cell of $Y$. It is contained in a unique $B$-segment $U$ of $Y$ and a unique member $W$ of $J$.

Consider an atom $Z$ of $M \cdot(E-Y)$ on $B$ determining a partition $\{S, T\}$ of $Y$, where $a \epsilon S$. Since $S \cup Z$ is an atom of $M \times(B \cup Y)$, by 8.12 , unless $S=Y$, it follows that $W \subseteq S$. Since this result must hold for all choices of $Z$ we have $W \subset U$.

Suppose $W$ is not the whole of $U$. Then there is an atom $X$ of $M \times(B \cup Y)$ which cuts $U$, in the sense of section 4.3. Choose such an $X$ so that $|B \cap X|$ has the least possible value.

Assume $B \cap X$ is not an atom of $(M \times(B \cup Y)) \cdot B$. Then there is an atom $Z$ of this matroid such that $Z \subseteq B \cup X$, and there is an atom $X^{\prime}$ of $\boldsymbol{M} \times(B \cup Y)$ such that $X^{\prime} \cap B=Z$. By the choice of $X$ the set $X^{\prime}$ does not cut $U$.

Consider the $\bmod 2 \operatorname{sum} X+X^{\prime}$. Clearly it cuts $U$. But it is a union of disjoint atoms of $M \times(B \cup Y)$, by 
5.34 , and one of these must cut $U$. This is contrary to the choice of $X$.

We deduce that $B \cap X$ is in fact an atom of $(M \times(B \cup Y)) \cdot B$, that is $(M \cdot(E-Y)) \times B$, by 3.334 . Hence $B \cap X$ is an atom of $M \cdot(E-Y)$ on $B$. But then $X$ does not cut $U$, by the definition of a $B$-segment.

From this contradiction it follows that in fact $W=U$. Since this result holds for all choices of $a$ the theorem follows.

8.53 Let $B$ be a bridge of $Y$ in $M$, and $S$ a subset of $E$ such that $B \cup Y \subseteq S$. Then $B$ is a bridge of $Y$ in $M \times S$, and

$$
\pi(M \times S, B, Y)=\pi(M, B, Y) .
$$

Proof: We have

$$
(M \times S) \cdot(S-Y)=(M \cdot(E-Y)) \times(S-Y),
$$

by 3.334. Hence $B$ is a separator of $(M \times S) \cdot(S-Y)$, by 3.43 . Moreover

$$
((M \cdot(E-Y)) \times(S-Y)) \times B=(M \cdot(E-Y)) \times B,
$$

by 3.331 , and this matroid is connected. Hence $B$ is a bridge of $Y$ in $M \times S$. Since

$$
(M \times S) \times(B \cup Y)=M \times(B \cup Y),
$$

by 3.331 , the theorem now follows from 8.52 .

8.531 Let $B$ be a bridge of $Y$ in $M$. Let $S$ be a subset of $E$ such that $B \cup Y \subset S$ and no $Z \epsilon M \cdot(E-Y)$ is a subset of $E-S$. Then $Y \epsilon M \cdot S, B$ is a bridge of $Y$ in $M \cdot S$, and

$$
\pi(M \cdot S, B, Y)=\pi(M, B, Y) .
$$

Proof: We have

$$
(M \cdot S) \cdot(S-Y)=(M \cdot(E-Y)) \cdot(S-Y),
$$

by 3.332. Hence $B$ is a separator of $(M \cdot S) \cdot(S-Y)$, by 3.43 . Moreover

$$
((M \cdot S) \cdot(S-Y)) \cdot B=(M \cdot(E-Y)) \cdot B,
$$

by 3.332 , and this matroid is connected. Hence $B$ is a bridge of $Y$ in $M \cdot S$, provided that $Y$ is an atom of $M \cdot S$.

Suppose some atom $X$ of $M \times((E-S) \cup B \cup Y)$ meets $E-S$. Then some $Z \epsilon M \cdot(E-Y)$ meets $E-S$ and satisfies

$$
Z \subseteq X \cap(E-Y) \subseteq(E-S) \cup B,
$$

by 1.11. But then $Z \subseteq E-S$, since $B$ is a separator of $M \cdot(E-Y)$, and this is contrary to the definition of $S$. We deduce that

$$
\begin{gathered}
(M \cdot S) \times(B \cup Y) \\
=(M \times((E-S) \cup B \cup Y)) \cdot(B \cup Y),
\end{gathered}
$$

by 3.333 ,

$$
\begin{aligned}
& =(M \times(B \cup Y)) \cdot(B \cup Y) \\
& =M \times(B \cup Y) .
\end{aligned}
$$

We note that this implies $Y \epsilon M \cdot S$. An application of 8.52 completes the proof.

8.54 Let $B$ be any bridge of $Y$ in $M$. Let $S$ be a subset of $E$ such that $E-Y \subset S$. Then $S \cap Y \in M \cdot S, B$ is a bridge of $S \cap Y$ in $M \cdot S$, and

$$
\pi(M \cdot S, B, S \cap Y)
$$

is the class of all non-null intersections with $S$ of members of $\pi(M, B, Y)$.

Proof: There exists $Y^{\prime} \epsilon M \cdot S$ such that $Y^{\prime} \subseteq S \cap Y$. Moreover there exists $Y^{\prime \prime} \epsilon M$ such that $Y^{\prime \prime} \cap S=Y^{\prime}$. But then $Y^{\prime \prime} \subseteq Y$. Hence $Y^{\prime \prime}=Y$ and $S \cap Y=Y^{\prime} \in M \cdot S$, by Axiom I.

We have

$$
(M \cdot S) \cdot(S-(S \cap Y))=M \cdot(S-(S \cap Y))=M \cdot(E-Y),
$$

by 3.332. Hence $B$ is a bridge of $S \cap Y$ in $M \cdot S$. Moreover

$$
\begin{aligned}
& ((M \cdot S) \times(B \cup(S \cap Y))) \cdot(S \cap Y) \\
= & (M \times(E-(S-(B \cup Y)))) \cdot S \cap Y) \\
= & ((M \times(B \cup Y)) \cdot Y) \cdot(S \cap Y),
\end{aligned}
$$

by 3.332 and 3.333 . The theorem now follows from 8.51 and 8.52 .

8.55 Let $B_{1}$ and $B_{2}$ be distinct bridges of $Y$ in $M$. Let $Z$ be a point of $M \cdot(E-Y)$ on $B_{1}$ determining $a$ partition $\{S, T\}$ of $Y$ where $S$ and $T$ are non-null. Let $Y^{\prime}$ be the point $S \cup Z$ of $M^{\prime}$ Then the following propositions hold.

(i) $T$ is an atom of $M \cdot\left(E-Y^{\prime}\right)$.

(ii) There is a bridge $B^{\prime}$ of $Y^{\prime}$ in $M$ such that $B_{2} \subseteq B^{\prime}$.

(iii) If $T$ is contained in some $W \in \pi\left(M, B_{2}, Y\right)$, then $B_{2}=B^{\prime}$. Moreover $\pi\left(M, B^{\prime}, Y^{\prime}\right)$ differs from $\pi(M$, $\left.B_{2}, Y\right)$ only in the replacement of $W$ by $(W-T) \cup Z$.

(iv) If $T$ is contained in no member of $\pi\left(M, B_{2}, Y\right)$, then $T \subseteq B^{\prime}$. Moreover each member of $\pi\left(M, B^{\prime}, Y^{\prime}\right)$ is then contained either in $Z$ or in a member of $\pi\left(M, B_{2}\right.$, $Y$ ).

Proof: Since $Z \cup T$ is an atom of $M$ there is an atom $T^{\prime}$ of $M \cdot\left(E-Y^{\prime}\right)$ such that $T^{\prime \prime} \subseteq T$. There is an atom $X$ of $M$ such that $X \cap\left(E-Y^{\prime}\right)=T^{\prime}$. But then $X$ is a point of $M$ on the line $Y^{\prime} \cup T=Y \cup Z$. Since the only points on this line are $Y, S \cup Z$ and $T \cup Z$ we have $X=T \cup Z$ and $T^{\prime}=T$. This establishes (i).

We have

$$
\left(M \cdot\left(E-Y^{\prime}\right)\right) \cdot B_{2}=(M \cdot(E-Y)) \cdot B_{2},
$$

by 3.332. As the expression on the right represents a connected matroid we deduce that $B_{2}$ is contained 
in some elementary separator $B^{\prime}$ of $M \cdot\left(E-Y^{\prime}\right)$, by 3.46. Then $B^{\prime}$ is a bridge of $Y^{\prime}$ in $M$ such that $B_{2} \subseteq B^{\prime}$. This establishes (ii).

To prove (iii) assume that $T$ is contained in some $W \epsilon \pi\left(M, B_{2}, Y\right)$. Let $Z_{1}$ be any atom of $M \cdot\left(E-Y^{\prime}\right)$ on $B^{\prime}$ which meets $B_{2}$.

There is an atom $X$ of $M \times\left(B^{\prime} \cup Y\right)$ such that $X \cap B^{\prime}$ $=Z_{1}$. Thus $X$ is an atom of $M$ meeting $B_{2}$. It follows that there is an atom $Z_{2}$ of $M \cdot(E-Y)$ such that $Z_{2} \cap B_{2}$ is non-null and $Z_{2} \subseteq X \cap(E-Y)$, by 1.11. Clearly $Z_{2}$ is on $B_{2}$. We thus have

$$
Z_{2} \subseteq Z_{2} \cap B^{\prime} \subseteq X \cap B^{\prime}=Z_{1}
$$

$Z_{2}$ determines a partition $\left\{S_{2}, T_{2}\right\}$ of $Y$, and by our assumption we may suppose $T \subseteq T_{2}$. The set $S_{2} \cup Z_{2}$ is an atom of $M$ which meets $B_{2}$ and therefore $B^{\prime}$. Hence there is an atom $Z_{3}$ of $M \cdot\left(E-Y^{\prime}\right)$ on $B^{\prime}$ such that $Z_{3} \subseteq\left(Z_{2} \cup S_{2}\right) \cap\left(E-Y^{\prime}\right)$. But $S_{2}$ does not meet $E-Y^{\prime}$ since $T \subset T_{2}$. Hence $Z_{3} \subseteq Z_{2} \subseteq Z_{1}$.

Applying Axiom I to $M \cdot\left(E-Y^{\prime}\right)$ we find that $Z_{1}=Z_{2}=Z_{3}$. We deduce that $B_{2}$ is a separator of $\left(M \cdot\left(E-Y^{\prime}\right)\right) \times B^{\prime}$. Hence $B_{2}=B^{\prime}$, by 3.44 . We note further that

$$
\begin{aligned}
& \left(M \cdot\left(E-Y^{\prime}\right)\right) \times B_{2} \\
= & \left(M \cdot\left(E-Y^{\prime}\right)\right) \cdot B_{2} \\
= & (M \cdot(E-Y)) \cdot B_{2} \\
= & (M \cdot(E-Y)) \times B_{2},
\end{aligned}
$$

by 3.332 and 3.42 . Hence $M \cdot(E-Y)$ and $M \cdot\left(E-Y^{\prime}\right)$ have the same atoms on $B_{2}$.

If one such atom $Z_{0}$ determines a partition $\left\{S_{0}, T_{0}\right\}$ of $Y$, with $T \subseteq T_{0}$, then it determines the partition $\left\{S_{0},\left(T_{0}-T\right) \cup Z\right\}$ of $Y^{\prime}$. For the point $S_{0} \cup Z_{0}$ is on both $Y \cup Z_{0}$ and $Y^{\prime} \cup Z_{0}$. These results establish (iii).

Now let us assume that $T$ is contained in no member of $\pi\left(M, B_{2}, Y\right)$, and discuss proposition (iv).

There is an atom of $M \times\left(B_{2} \cup Y\right)$ which cuts $T$ in the .sense of section 4.3. Hence there is an atom $Z_{1}$ of $M \cdot\left(E-Y^{\prime}\right)$ which cuts $T$ in this sense and satisfies $Z_{1} \subseteq\left(B_{2} \cup Y\right) \cap\left(E-Y^{\prime}\right) \subseteq B^{\prime} \cup T$. Since $T \epsilon M \cdot\left(E-Y^{\prime}\right)$, by (i), it follows that $\bar{Z}_{1}$ meets both $B^{\prime}$ and $T$. Hence $T \subseteq B^{\prime}$, by 3.48 .

Now $Z$ is the intersection of $Y^{\prime}$ with an atom $Z \cup T$ of $M \times\left(B^{\prime} \cup Y^{\prime}\right)$. Hence it is a union of members of $\pi\left(M, B^{\prime}, Y^{\prime}\right)$ by 8.51 and 8.52 .

Again, let $Z_{0}$ be any atom of $M \cdot(E-Y)$ on $B_{2}$, determining a partition $\left\{S_{0}, T_{0}\right\}$ of $Y$. Then $S \cap S_{0}$ is the intersection with $Y^{\prime}$ of the atom $S_{0} \cup Z_{0}$ of $M \times\left(B^{\prime} \cup Y^{\prime}\right)$, since $T \subseteq B^{\prime}$. Hence $S \cap S_{0}$ and similarly $S \cap T_{0}$ are unions of members of $\pi\left(M, B^{\prime}, Y\right)$, by 8.51 and 8.52. Since this is true for each choice of $Z_{0}$ proposition (iv) follows.

\subsection{Regular Matroid}

We conclude this chapter with some theorems about binary matroids with no minors of one of the types BI and BII. These are all true for regular matroids, by 7.51 .

8.61 Suppose $M$ has no minor of Type BI. Let $Z_{1}$ and $Z_{2}$ be points on a connected line $L$ of $M \cdot(E-Y)$. Then $Z_{1}$ and $Z_{2}$ are not skew with respect to $Y$.

Proof: $L \cup Y$ is a plane $P$ of $M$, by 8.12. It includes three distinct lines $Y \cup Z_{1}, Y \cup Z_{2}$, and $Y \cup Z_{3}$ of $M$, where $Z_{3}$ is the third point of $M \cdot(E-Y)$ on $L$, by 8.12 .

Assume $Z_{1}$ and $Z_{2}$ skew. They determine partitions $\left\{S_{1}, T_{1}\right\}$ and $\left\{S_{2}, T_{2}\right\}$ of $Y$ respectively. There are cells $a \epsilon S_{1} \cap S_{2}, b \epsilon S_{1} \cap T_{2}, c \epsilon T_{1} \cap S_{2}$, and $d \epsilon T_{1} \cap T_{2}$. The flats $\langle P-\{a\}\rangle,\langle P-\{b\}\rangle,\langle P-\{c\}\rangle, \quad$ and $\langle P-\{d\}\rangle$ are lines on $P$ which are not on $Y$, by 4.13 . It is easily seen that they are distinct. For example $\langle P-\{a\}\rangle$ is the only one which is on the two points $Z_{1} \cup T_{1}$ and $Z_{2} \cup T_{2}$ of $M$.

We see that there are seven distinct lines on $P$. Thus $M$ includes a Fano configuration, which is contrary to hypothesis, by 7.21 .

8.62 Suppose $M$ has no minor of Type BI. Let B be any non-trivial bridge of $Y$ in $M$, and let $W$ be any member of $\pi(M, B, Y)$. Then there exists $Z \epsilon M \cdot(E-Y)$ on $B$ determining the partition $\{W, Y-W\}$ of $Y$. (Compare 8.43).

Proof: Let $a$ be any cell of $Y$. Let $U$ be a subset of $Y$ such that $a \epsilon U$, some $Z \epsilon M \cdot(E-Y)$ on $B$ determines the partition $\{U, Y-U\}$ of $Y$, and $|U|$ has the least value consistent with these conditions.

Assume there is a $W \epsilon \pi(M, B, Y)$ such that $W \cap U$ is a non-null proper subset of $U$. Then there exists $X \epsilon M \times(B \cup Y)$ such that $X \cap U$ is a non-null proper subset of $U$. Clearly $X \cup Y$ is a connected flat of $M \times(B \cup Y)$. By 4.31 and 4.34 we can find a point $X^{\prime}$ of $M \times(B \cup Y)$ on $X \cup Y$ which cuts $U$ in the sense of section 4.3 and has the property that $X^{\prime} \cup Y$ is a connected line of $M \times(B \cup Y)$. Then $X^{\prime}-Y$ is a point of $M \cdot(E-Y)$ on $B$, by 8.12 . It determines the partition $\left\{X^{\prime} \cap Y, Y-X^{\prime}\right\}$ of $Y$, and so cuts $U$ in the sense of section 8.4.

By 4.34 and 8.41 we can find $Z^{\prime} \epsilon M \cdot(E-Y)$ on $B$ which cuts $U$ and is such that $Z \cup Z^{\prime}$ is a connected line of $M \cdot(E-Y)$. By $8.61 Z^{\prime}$ determines a partition $\{S, Y-S\}$ of $Y$ such that $S \subset U$. By the definition of $U$ we have $a \epsilon U-S$.

Choose $b \epsilon S$. By 8.12 the set $Y \cup Z \cup Z^{\prime}$ is a plane $P$ of $M$. The line $\langle P-\{b\}\rangle$ of $M$ is on the points $Z \cup(Y-U)$ and $Z^{\prime} \cup(Y-S)$ of $M$. Since these are distinct and not disjoint their $\bmod 2$ sum, $Q$ say, is also a point of $M$ on $\langle P-\{b\}\rangle$, by 5.34 and 5.35. But the $\bmod 2$ sum of $Z$ and $Z^{\prime}$ is the third point of $M \cdot(E-Y)$ on the connected line $Z \cup Z^{\prime}$. It determines the partition

$$
\{Q \cap Y, Y-Q\}=\{U-S, Y-(U-S)\}
$$

of $Y$. But this contradicts the definition of $U$.

We deduce from this contradiction that $U$ is itself a member of $\pi(M, B, Y)$. Since $a$ may be any cell of $Y$ the theorem follows.

8.63 Suppose $M$ has no minor of Type BI, and that $d M \geqslant 2$. Then there is an atom $Y$ of $M$ which has two distinct bridges in $M$. 
Proof: Assume the theorem false. Choose $X \epsilon M$. Then $E-X$ is a bridge of $X$ in $M$. It is nontrivial since $d M \geqslant 2$.

Choose $W \epsilon \pi(M, E-X, X)$. There exists $Z \epsilon M \cdot(E-X)$ determining the partition $(W, X-W)$ of $X$, by 8.62 . Then $X \cup Z$ is a line of $M$, possibly disconnected, and $Z \cup(X-W),=Y$ say, is a point of $M$. Since $d M \geqslant 2$ the set $E-(X \cup Z)$ is non-null.

$W$ is a point of $M \cdot(E-Y)$, by 8.55. Suppose $Z^{\prime} \in M \cdot(E-Y)$ and $Z^{\prime} \cap W$ is non-null. Then there exists $V \epsilon M$ such that $Z^{\prime}=V \cap(E-Y)$. Then $V \cap W$ $=Z^{\prime} \cap W$. Hence $W \subseteq Z^{\prime}$ since $W \epsilon \pi(M, E-X, X)$, and therefore $W=Z^{\prime}$ by Axiom $\mathrm{I}$.

It follows that $W$ is a separator of $M \cdot(E-Y)$. But $(E-Y)-W=E-(Y \cup Z) \neq \phi$. Hence there are at least two bridges of $X$ in $M$, one being $W$.

8.64 Suppose $M$ has no minor of Type BII. Let $Z_{1}, Z_{2}$ and $Z_{3}$ be points of $M \cdot(E-Y)$ on distinct bridges $B_{1}$, $B_{2}$ and $B_{3}$ respectively of $Y$ in $M$. Let $Z_{i}$ determine the partition $\left\{S_{i}, T_{i}\right\}$ of $Y . \quad(i=1,2,3)$. Then if $S_{3}$ meets both $S_{1} \cap S_{2}$ and $T_{1} \cap T_{2}$ it contains one of the sets $S_{1} \cap T_{2}$ and $S_{2} \cap T_{1}$.

Proof: Suppose the theorem false. Then we can choose $K=\{a, b, c, d, e, f, g\} \subseteq E$ such that $a \in Z_{1}, b \in Z_{2}$, $c \epsilon Z_{3}, d \epsilon S_{1} \cap S_{2} \cap S_{3}, e \epsilon T_{1} \cap T_{2} \cap S_{3}, f \epsilon\left(S_{1} \cap T_{2}\right)-S_{3}$, and $g \in\left(S_{2} \cap T_{1}\right)-S_{3}$.

In the matroid $M \cdot(E-Y)$ the sets $Z_{1} \cup Z_{2}, Z_{2} \cup Z_{3}$, and $Z_{3} \cup Z_{1}$ are disconnected lines, by 4.24. Moreover $Z_{1} \cup Z_{2} \cup Z_{3}$ is a plane since there are only three disjoint points on it. $\quad\left(\{a, b, c\}\right.$ is a dendroid of $M \times\left(Z_{1} \cup Z_{2}\right.$ $\left.\cup Z_{3}\right)$ ). Hence $Y \cup\left(Z_{1} \cup Z_{2} \cup Z_{3}\right)$ is a 3 -flat $J$ of $M$, by 8.12 .

There are points of $M$ on $J$ whose intersections with $K$ are $\{a, d, f\},\{a, g, e\},\{b, d, g\},\{b, e, f\},\{c, f, g\}$, $\{c, d, e\}$, and $\{a, b, c\}$. For example $\left(Z_{1} \cup S_{1}\right) \cap K$ $=\{a, d, f\}$, and similar verifications may be made for the next five intersections. As for $\{a, b, c\}$ we observe that

$Z_{1} \cup T_{1} \subset Z_{1} \cup T_{1} \cup Z_{2} \cup T_{2}$

$\subset Z_{1} \cup T_{1} \cup Z_{2} \cup T_{2} \cup Z_{3} \cup T_{3} \subset J$.

Hence $Z_{1} \cup T_{1} \cup Z_{2} \cup T_{2}$ is a line of $M$, by 4.14 . Its points are $Z_{1} \cup T_{1}, Z_{2} \cup T_{2}$ and their mod 2 sum, $Q$ say. We have $Q \cap K=\{a, b, f, g\}$. Moreover $Z_{1} \cup T_{1} \cup Z_{2} \cup T_{2} \cup Z_{3} \cup T_{3}$ is a plane $P$ of $M$ on $E$, by 4.14. The line $\langle P-\{e\}\rangle$ of $M$ is on the points $Z_{3} \cup T_{3}$ and $Q$. As these two distinct points have a common cell $f$ their $\bmod 2$ sum, $R$ say, is the third point of $M$ on $\langle P-\{e\}\rangle$. But $R \cap K=\{a, b, c\}$.

Considering the seven intersections with $K$ set out above we see that each cell of $K$ occurs in just three of them and that no two have two cells in common. Hence given any three cells $x, y$, and $z$ of $K$ we can find a point of $M$ on $J$ which includes $x$ but not $y$ or $z$. It follows that no three of the planes $\langle J-\{x\}\rangle, x \in K$, have a common line; we can find a point on any two of them which is not on the third.

Hence these seven planes on $J$ are distinct and define a heptahedron. This is contrary to hypothesis, by 7.31 .

\section{Graphic Matroids}

\subsection{Bonds}

We consider the group $N$ of coboundaries of a graph $G$ over a commutative ring $R$, with a unit element and no divisors of zero. As explained in section 2.5 $M(N)$ is the bond-matroid $B(G)$ of $G$, and is independent of $R$.

9.11 Let $f$ be any 0-chain on $G$ over $R$. Let $H$ be any component of the spanning subgraph $G:(E(G)-\|\delta f\|$ of G. (See sec. 3.1.) Then the vertices of $H$ have equal coefficients in $f$.

Proof: Suppose the contrary. Then some edge $A$ of $H$ has ends with different coefficients in $f$. But then $A \epsilon\|\delta f\|$, by the definition of a coboundary, which is contrary to the definition of $H$.

9.12 Let $g$ be an elementary chain of $N$. Then there are distinct components $H$ and $K$ of $G:(E(G)-\|g\|)$ such that each edge of $\|g\|$ has one end in $V(H)$ and one in $V(K)$.

Proof: Write $g=\delta f$. Let $A$ be any edge of $\|g\|$. Its ends $x$ and $y$ have unequal coefficients $h$ and $k$ respectively in $f$. Let $H$ and $K$ be the components of $G:(E(G)-\|g\|)$ containing $x$ and $y$ respectively. They are distinct, by 9.11 .

Let $f_{H}$ be the 0 -chain in which the members of $V(H)$ have coefficient 1 and all other vertices of $G$ have coefficient 0 . Then $\left\|\delta f_{H}\right\| \subseteq\|\delta f\|=\|g\|$ and therefore $\left\|\delta f_{H}\right\|=\|g\|$ since $g$ is elementary. We deduce that each edge of $\|g\|$ has one end in $V(H)$ and similarly one end in $V(K)$.

9.13 A set $Y \subseteq E(G)$ is an atom of $M(N)$ if and only if there are components $H$ and $K$ of $G:(E(G)-Y)$ such that each edge of $Y$ has one end in $V(H)$ and one in $V(K)$.

Proof: If $\dot{Y} \epsilon M(N)$ the above condition is satisfied, by 9.12 .

Conversely suppose $G:(E(G)-Y)$ has components $H$ and $K$ with the stated property. Clearly $Y$ is the domain of a coboundary $\delta f$ such that the vertices of $H$ have coefficient 1 in $f$, and those of $V(G)-V(H)$ coefficient 0 .

Suppose however that there is a coboundary $\delta f_{1}$ of $G$ such that $\left\|\delta f_{1}\right\|$ is a proper subset of $Y$. Then there is a component $J$ of $G:\left(E(G)-\left\|\delta f_{1}\right\|\right.$ which contains both $H$ and $K$. Hence $\delta f_{1}=0$, by 9.11 and the definition of a coboundary.

Theorem 9.13 provides another proof that $M(N)$ is independent of $R$. Its atoms are the bonds of $G$.

If $Y$ is any bond of $G$ then the graphs $H$ and $K$ of 9.13 are the end-graphs of $Y$ in $G$.

If $S \subseteq E(G)$ the common vertices of $G \cdot S$ and $G$. $(E(G)-S)$ are the vertices of attachment of $S$ (and of $E(G)-S)$ in $G$. We denote their number by $w(S)$. They may also be defined as those vertices of $G$ which are incident with edges of both $S$ and $E(G)-S$. If $w(S)=1$ then the single vertex of attachment of $S$ is called a cut-vertex of $G$.

Let $x$ be any vertex of $G$. The star $S t(x)$ of $x$ in $G$ is the set of all edges having one end at $x$ and one in 
$V(G)-x$. We also write $L(x)$ for the set of all edges (loops) of $G$ whose ends coincide at $x$.

The remaining theorems of this section relate the structure of $G$ to that of $B(G)$.

9.14 Let $S$ be a subset of $E(G)$ such that $w(S) \leqslant 1$. Then $S$ is a separator of $B(G)$.

Proof: Let $Y$ be any bond of $G$, with end graphs $H$ and $K$. We may suppose $K$ to include no common vertex of $G \cdot S$ and $G \cdot(E(G)-S)$. Then the intersections of $K$ with $G \cdot S$ and $G \cdot(E(G)-S)$ have no common edge or vertex, and since $K$ is connected one of them is null. Hence either $V(K) \subseteq V(G \cdot S)$ or $V(K) \subseteq V(G \cdot(E(G)-S))$, whence it follows that either $Y \subseteq S$ or $Y \subseteq E(G)-S$. The theorem follows.

As a corollary we have

\subsection{If a is a loop of $G$ then $\{a\}$ is a separator of $B(G)$.}

Such a separator may be called trivial since it meets no atom of $B(G)$.

9.15 Let $x$ be any vertex of $G$ such that $S t(x)$ is non-null, and let $\mathrm{G}_{1}$ be the graph derived from it by deleting the loops of $L(x)$. Then $S t(x)$ is a bond of $G$ if and only if $x$ is not a cut-vertex of $G_{1}$.

Proof: Let $G_{2}$ be the graph obtained from $G$ by deleting the vertex $x$ and its incident edges. Let those components of $G_{2}$ which include vertices incident with members of $S t(x)$ in $G$ be enumerated as $H_{1}, H_{2}, \ldots ., H_{k}$. Such components exist since $S t(x)$ is non-null. (See fig. 9A.)

Clearly $x$ is a cut-vertex of $G_{1}$ if and only if $k \geqslant 2$.

But the set of edges of $S t(x)$ incident in $G$ with members of $V\left(H_{i}\right)$ is a bond of $G$ for each $i$, by 9.13 , one of its end-graphs being $H_{i}$. Hence $S t(x)$ is a bond of $G$ if $k=1$. But if $k \geqslant 2$ then $\operatorname{St}(x)$ has a bond of $G$ as a proper subset, and is not itself an atom of $B(G)$, by Axiom I.

$9.16 B(G)$ is a connected matroid if and only if $G \cdot E(G)$ is connected and $G$ has no cut-vertex.

Proof: If $G \cdot E(G)$ is not connected or if $G$ has a cut-vertex it is clear that some non-null proper subset $S$ of $E(G)$ satisfies $w(S) \leqslant 1$. Then $S$ is a separator of $B(G)$, by 9.14 .

Conversely suppose $B(G)$ is not a connected matroid. Then there is a proper non-null subset $S$ of $E(G)$ which is a separator of $B(G)$.

Suppose $G$ has a loop $A$ incident with a vertex $x$. Then either $x$ is a cut-vertex of $G$, or $G \cdot\{A\}$ is a component of $G \cdot E(G)$. In either case the theorem is satisfied.

We may now assume that $G$ as no loop or cut-vertex. Let $x$ be any vertex of attachment of $S$. Then $S t(x)$ is an atom of $B(G)$ meeting both $S$ and $E(G)-S$, by 9.15 , which contradicts the definition of $S$ as a separator. We conclude that $w(S)=0$. Hence $G \cdot S$ is a union of components of $G \cdot E(G)$. This completes the proof of the theorem.

9.17 Let $S$ be a non-null subset of $E(G)$. Then $S$ is an elementary separator of $B(G)$ if and only if the following conditions hold.

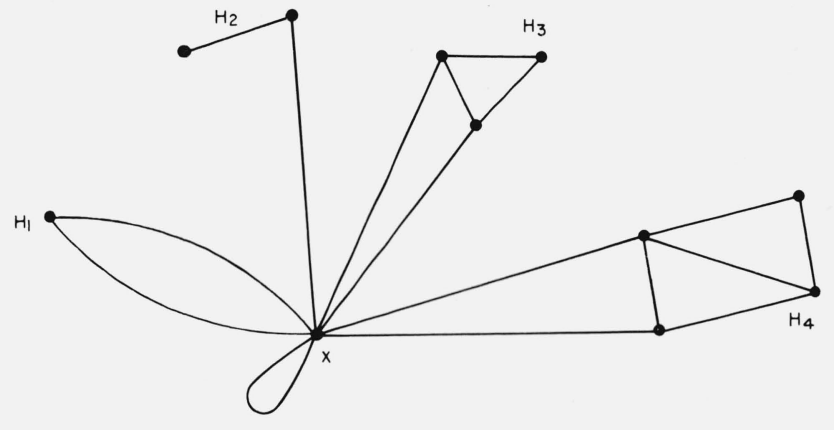

Figure 9A

(i) $G \cdot S$ is connected and has no cut-vertex.

(ii) Each component of $G$ : $(E(G)-S)$ has at most one vertex in common with $G \cdot S$.

Proof: Suppose first that $S$ is an elementary separator of $B(G)$. Then $B(G) \cdot S$ is a connected matroid, by 3.47. But $B(G) \cdot S=B(G \cdot S)$, by 3.321 . Hence $G \cdot S$ is connected and has no cut-vertex, by 9.16.

It may happen that $S$ consists of a single loop of $G$. In that case proposition (ii) is trivially true. We may therefore assume that no edge of $S$ is a loop.

Let $x$ be any vertex of $G \cdot S$, and let $T$ be its star in $G \cdot S$. Then $T$ is an atom of $B(G \cdot S)$, of $B(G) \cdot S$, of $B(G) \times S$ and $B(G)$, by 3.321 and 3.42 . Let $C(x)$ be its end-graph in $G$ which includes $x$. Clearly $C(x)$ is a component of $G:(E(G)-S)$, and $x$ is its only common vertex with $G \cdot S$.

Conversely suppose $S$ to satisfy (i) and (ii). If $C$ is any component of $G:(E(G)-S)$ then $w(E(C)) \leqslant 1$, by (ii), and therefore $E(C)$ is a separator of $B(G)$, by 9.14 . The union of all such sets $E(C)$, namely $E(G)-S$, is thus also a separator of $B(G)$. Hence $S$ is a separator of $B(G)$.

If $S$ is not an elementary separator of $B(G)$ there is another non-null separator $S^{\prime}$ of $B(G)$ such that $S^{\prime} \subset S$. But then $S^{\prime}$ is a separator of $B(G \cdot S)$, by 3.321 and 3.43. Hence (i) is contradicted, by 9.16.

This completes the proof.

Suppose we are given an elementary separator $S$ of $B(G)$. For each vertex $x$ of $G \cdot S$ we define $C(G, S, x)$ as that component of $G:(E(G)-S)$ which has $x$ as a vertex. If $x$ and $y$ are distinct vertices of $G \cdot S$ then $C(G, S, x)$ and $C(G, S, y)$ have no common vertex, by 9.17 .

9.18 Let $G$ be a connected graph, and let $S$ and $T$ be distinct elementary separators of $B(G)$. Then there are vertices $s$ and $t$, of $G \cdot S$ and $G \cdot T$, respectively, such that $G \cdot S$ is a subgraph of $C(G, T, t)$, and $G \cdot T$ is a subgraph of $C(G, S, s)$. Moreover each vertex of $G$ belongs to one or both of $C(G, T, s)$ and $C(G, T, t)$.

Proof: The first part of the theorem follows from the fact that $G \cdot S$ is a connected subgraph of $G$ : $(E(G)-T)$, by 9.17 , and from the analogous result for $G \cdot T$.

To prove the second part suppose $v$ is a vertex of $G$ not belonging to $C(G, S, s)$. Then, by the connection 


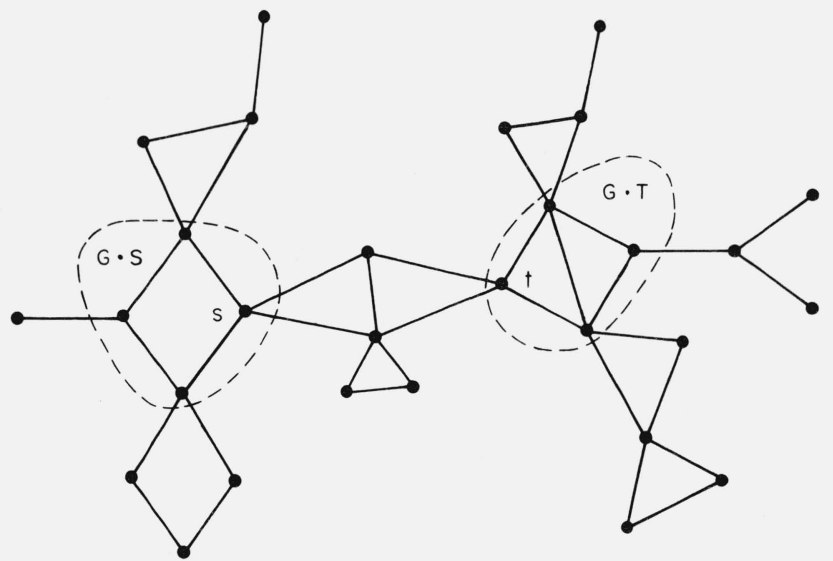

FiguRE 9B

of $G, v$ belongs to some $C(G, S, u)$ such that $u \neq s$ and $C(G, S, u)$ has no common vertex with $G \cdot T$. But then $c(G, S, u)$, with $G \cdot S$, is a subgraph of $C(G, T, t)$. (See fig. 9B.)

\subsection{Bridges in a Bond-Matroid}

Let $Y$ be a bond in a graph $G$, with end-graphs $H$ and $K$. Write $G_{1}=G \cdot(E(G)-Y)$.

If $S$ is an elementary separator of $B\left(G_{1}\right)$, that is $B(G) \cdot(E(G)-Y)$, then the connected graph $G_{1} \cdot S$ is a subgraph of some component of $G_{1}$. We refer to $G_{1} \cdot S$, which is identical with $G \cdot S$, as a bridge-graph of $Y$ in $G$.

We note that any component of $G_{1}$ either consists solely of an isolated vertex, or it is a union of bridgegraphs of $Y$ in $G$. In particular this observation applies to $H$ and $K$. It leads immediately to the following important theorem.

9.21 If $B(G) \cdot(E(G)-Y)$ is a connected matroid, then either $H$ or $K$ consists of a single vertex.

This result is often helpful in determining whether a given binary matroid is graphic.

Suppose $G \cdot S$ is a bridge-graph of $Y$ in $G$. Let $v$ be one of its vertices. We write $Y(S, v)$ for the set of all edges of $Y$ having one end in $C\left(G_{1}, S, v\right)$. The set $Y(S, v)$ can thus be non-null only if $G \cdot S$ is contained in an end-graph of $Y$ in $G$. (See fig. 9C.)

9.22 Let $S$ be a bridge of $Y$ in $B(G)$, so that $G \cdot S$ is a bridge-graph of $Y$ in $G$. Then either $\pi(B(G), S, Y)$ is $\{Y, \phi\}$ or it is the class of all non-null subsets $Y(S, v)$ of $Y$ such that $v \in V(G \cdot S)$.

Proof: Let $Z$ be any atom of $B(G) \cdot(E(G)-Y)$ on $S$. Then $Z$ is a bond of $G \cdot S$. Let its end-graphs in $G \cdot S$ be $H$ and $K$. These two subgraphs of $G$, together with the edges of $Z$ constitute the whole of $G \cdot S$, by 9.17 .

Let $H_{1}$ be the union of $H$ with all the graphs $C\left(G_{1}\right.$, $S, v)$ such that $v \epsilon V(H)$, and let $K_{1}$ be defined similarly.

It may happen that one of $H_{1}$ and $K_{1}$ has no vertex incident in $G$ with a member of $Y$. In that case $Z$ is

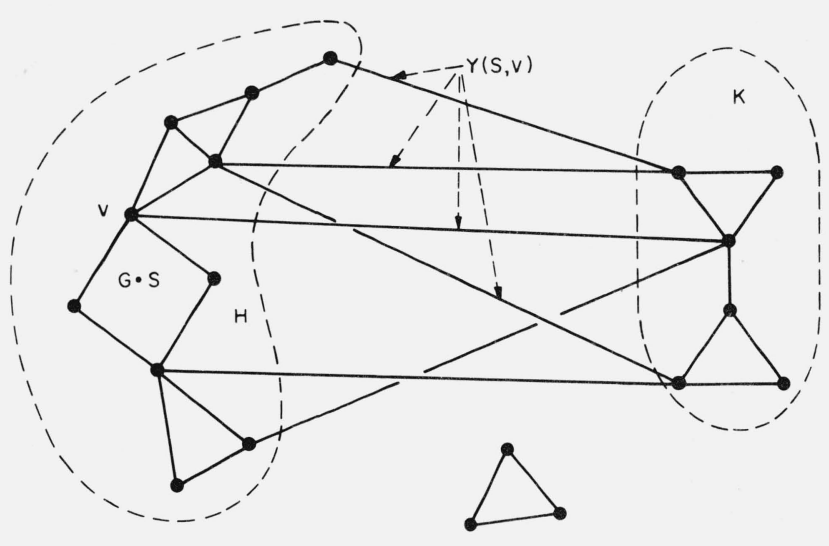

FIGURE 9C

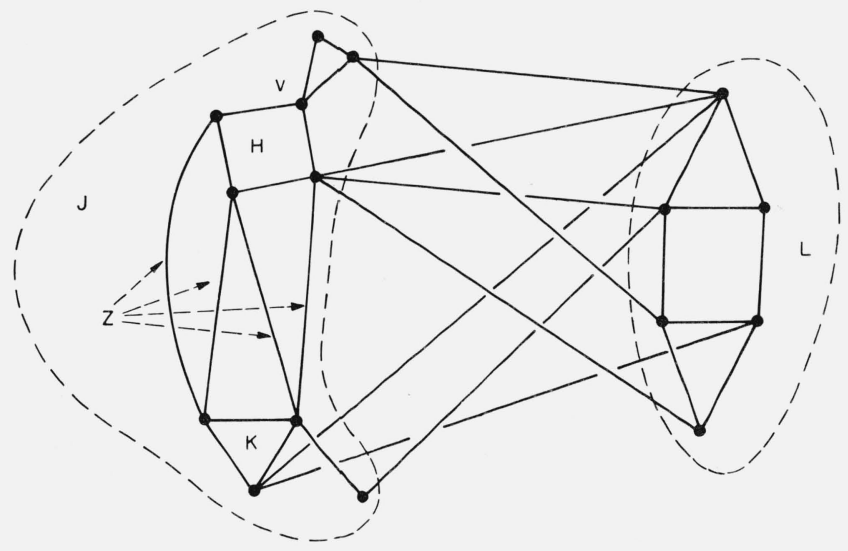

Figure 9D

a bond of $G$ with either $H_{1}$ or $K_{1}$ as one end-graph. It then determines the trivial partition $\{Y, \phi\}$ of $Y$.

In the remaining case each of $H_{1}$ and $K_{1}$ has a vertex incident with a member of $Y$. Then $G \cdot S$ is contained in an end-graph $J$ of $Y$ in $G$. Let the other end-graph of $Y$ in $G$ be $L$. (See fig. 9D.) Then $H_{1}$ is an end-graph of a bond $Y_{1}$ of $G$, whose other end-graph is made up of $L, K_{1}$ and the edges of $Y$ joining $L$ to $K_{1}$. We have

$$
Y_{1}=Z \cup\left[\cup_{v \in V(H)} Y(S, v)\right]
$$

Since this argument applies for each choice of $Z$ we deduce that each member of $\pi(B(G), S, Y)$ is a union of sets of the form $Y(S, v)$. The argument assumes $S$ to be a nontrivial bridge, but the result is still trivially true in the remaining case.

Consider any vertex $v$ of $G \cdot S$. If $S$ consists of a single loop of $G$ we have $Y=Y(S, v)$. In the remaining case $S$ includes no loop of $G$, by 9.17 . Let $Z$ be the star of $v$ in $G \cdot S$. Then $Z$ is a bond of $G \cdot S$ one of whose end-graphs consists solely of the vertex $v$, by 9.15 
and 9.17. It therefore determines a partition of $Y$ having $Y(S, v)$ as one of its primary segments, by the preceding result.

Combining the above results we find that $Y(S, v)$ is either null or a member of $\pi(B(G), S, Y)$, for each $v \epsilon V(G \cdot S)$, and hence that the theorem is true.

This theorem may be regarded as verifying 8.62 for graphic matroids.

9.23 Every graphic matroid is even.

Proof: Let $M$ be a graphic matroid. Then $M=B(G)$ for some graph $G$. Let $Y$ be any atom of $M$, that is any bond of $G$, and let the end-graphs of $Y$ in $G$ be $H$ and $K$.

Let $S_{1}$ and $S_{2}$ be overlapping bridges of $Y$ in $B(G)$. The bridge graphs $G \cdot S_{1}$ and $G \cdot S_{2}$ are each contained in an end-graph of $G$.

Suppose they are subgraphs of the same end-graph $H$. There are vertices $v_{1}$ of $G \cdot S_{1}$ and $v_{2}$ of $G \cdot S_{2}$ such that $G \cdot S_{2}$ is a subgraph of $C\left(G_{1}, S_{1}, v\right)$ and $G \cdot S_{1}$ is a subgraph of $C\left(G_{2}, S_{2}, v\right)$, by 9.18 , applied to $H$. Moreover

$$
Y=Y\left(S_{1}, v_{1}\right) \cup Y\left(S_{2}, v_{2}\right)
$$

by the same theorem. This contradicts the hypothesis that $S_{1}$ and $S_{2}$ overlap, by 9.22 .

We deduce that if $S_{1}$ and $S_{2}$ overlap then $G \cdot S_{1}$ and $G \cdot S_{2}$ are subgraphs of distinct end-graphs of $G$.

Let $P$ be the class of all bridges $S$ of $Y$ in $M$ such that $G \cdot S$ is a subgraph of $H$, and let $Q$ be the class of all other bridges of $Y$ in $M$. Then, by the result just proved no members of the same class $P$ or $Q$ overlap. Thus $Y$ is bridge-separable.

It follows that $M$ is an even matroid.

Our next theorem shows that two distinct graphs, even though without cut-vertices, can have identical bond-matroids.

Let $G$ be a graph and let $S$ be a subset of $E(G)$ such that $w(S)=2$. Let the vertices of attachment of $S$ be $x$ and $y$. We construct a graph $G^{\prime}$ such that $E(G)$ $=E\left(G^{\prime}\right), V(G)=V\left(G^{\prime}\right)$, and the same incidence relations hold in $G^{\prime}$ as in $G$, with the following exception. If $A \epsilon S$, then $A$ is incident with $x$ or $y$ in $G^{\prime}$ if and only if it is incident with $y$ or $x$ respectively in $G$. We say $G^{\prime}$ is obtained from $G$ by reversing $S$. We can recover $G$ from $G^{\prime}$ by reversing $S$ again. We illustrate the operation in figure $9 \mathrm{E}$.

$9.24 B(G)$ is invariant under the operation of reversing a subset $S$ of $G$ such that $w(S)=2$.

Proof: Let $g$ be the 0-chain of $G$ over $G F(2)$ such that $g(z)=1$ when $z$ is a vertex of $G \cdot S$ other than $x$ or $y$, and $g(z)=0$ in the remaining cases.

If $f$ is any 0 -chain on $G$ over $G F(2)$ it is readily verified that the coboundary of $f$ in $G$ is the coboundary of $f+g$ in $G^{\prime}$. Hence $G$ and $G^{\prime}$ have the same coboundary group over $G F(2)$. The theorem follows, by the definition of a bond-matroid.

9.25 Let $Y$ be a totally bridge-separable atom of $a$ connected graphic matroid $M$. Then there is a connected graph $G$, having no cut-vertex, such that $M$ $=B(G)$ and some end-graph of $Y$ in $G$ consists of a single vertex.
Proof: There exists $G$ such that $M=B(G)$. We may suppose $G$ to be without isolated vertices, since $M=B(G) \cdot E(G)=B(G \cdot E(G))$. Then $G$ is connected and has no cut-vertex, by 9.17. We write $G_{1}=$ $G \cdot(E(G)-Y)$ as before.

Let the end-graphs of $Y$ in $G$ be $H_{1}$ and $H_{2}$. By the connection of $G$ these are the only components of $G_{1}$. We suppose $G, H_{1}$, and $H_{2}$ chosen so that $\left|E\left(H_{2}\right)\right|$ has the least possible value.

Assume $\left|E\left(H_{2}\right)\right|>0$. Then also $\left|E\left(H_{1}\right)\right|>0$, by the above choice.

Choose bridge-graphs $G \cdot S_{1}$ and $G \cdot S_{2}$ of $Y$ in $G$ contained in $H_{1}$ and $H_{2}$ respectively. Since the bridges $S_{1}$ and $S_{2}$ do not overlap there are, by 9.22 , vertices $v_{1}$ and $v_{2}$ of $G \cdot S_{1}$ and $G \cdot S_{2}$ respectively such that

$$
Y\left(S_{1}, v_{1}\right) \cup Y\left(S_{2}, v_{2}\right)=Y .
$$

Keeping $S_{2}$ and $v_{2}$ fixed we consider all possible choices of $S_{1}$ and $v_{1}$ for which (i) is true, and we select one for which $C\left(G_{1}, S_{1}, v_{1}\right)$ has the least number of edges.

Consider the set of all bridges $S$ of $Y$ in $M$ such that $G \cdot S$ has $v_{1}$ as a vertex. (See fig. 9F.) Let them be enumerated as $P_{1}, P_{2}, \ldots, P_{k}$, taking $P_{1}=S_{1}$.
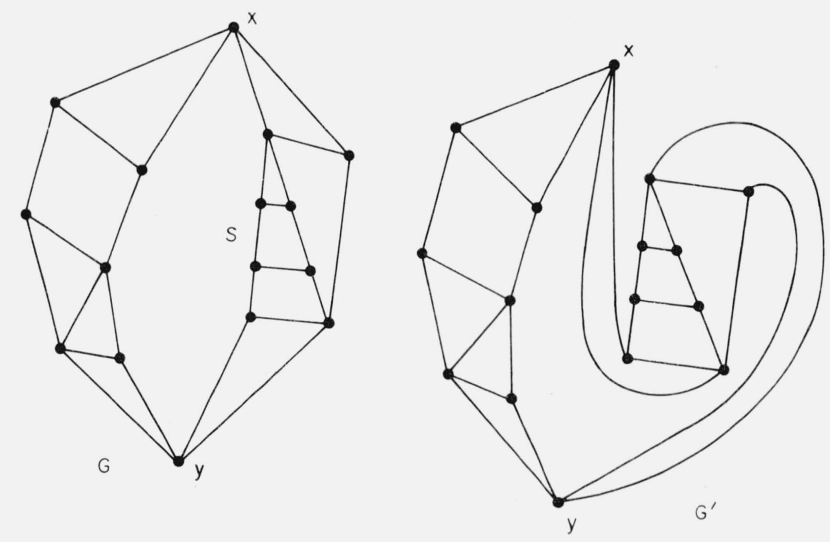

Figure 9E

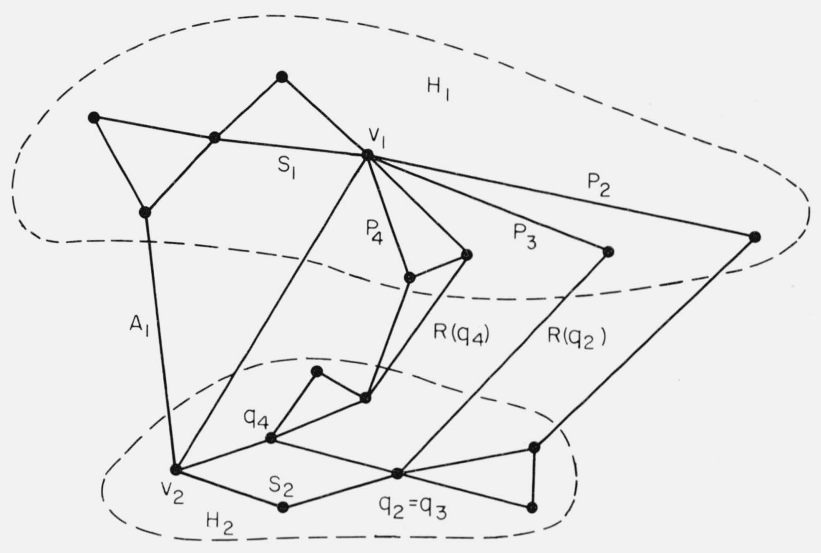

FiGURE 9F 
For each $P_{j}$ let $F_{j}$ be the subgraph of $H_{1}$ which is the union of $G \cdot P_{j}$ and those subgraphs $C\left(G_{1}, P_{j}, x\right)$ of $G_{1}$ for which $x \in V\left(G \cdot P_{j}\right)-\left\{v_{1}\right\}$. The graphs $F_{j}$ have the common vertex $v_{1}$, and each of them is connected. By 9.17 no two of them have any common vertex other than $v_{1}$. Since $H_{1}$ is connected it is the union of the graphs $F_{j}$.

For each $P_{j}$ there is a vertex $p_{j}$ of $G \cdot P_{j}$ and a vertex $q_{j}$ of $G \cdot S_{2}$ such that

$$
Y\left(P_{j}, p_{j}\right) \cup Y\left(S_{2}, q_{j}\right)=Y .
$$

This follows from the hypothesis that $Y$ is totally bridge-separable, and from 9.22. In accordance with (i) we take $p_{1}=v_{1}$ and $q_{1}=v_{2}$.

Since $G$ has no cut-vertex it follows that for each $P_{j}$ there is an edge $A_{j}$ in $Y\left(S_{2}, q_{j}\right)$ but not in $Y\left(P_{j}, p_{j}\right)$, and an edge $D_{j}$ in $Y\left(P_{j}, p_{j}\right)$ but not in $Y\left(S_{2}, q_{j}\right)$.

Suppose $p_{j} \neq v_{1}$. Considering the edge $A_{1}$, of which one end is a vertex of $F_{1}$ other than $v_{1}$, we find that $q_{j}=q_{1}=v_{2}$, by (ii). But in this case $C\left(G_{1}\right.$, $\left.P_{j}, p_{j}\right)$ is a subgraph of $C\left(G, S_{1}, v_{1}\right)$, and has fewer edges than $C\left(G_{1}, S_{1}, v_{1}\right)$. This however contradicts the definition of $S_{1}$ and $v_{1}$. We deduce that in fact

$$
p_{j}=v_{1}
$$

for each $j$.

Considering the edge $A_{j}$ we see that $q_{j}$ is uniquely determined for each $p_{j}$. Let $Z_{j}$ be the set of all edges of $Y$ having one end a vertex of $F_{j}$ other than $v_{1}$. Then $Z_{j}$ is non-null since it includes $A_{j}$. By (ii) each edge of $Z_{j}$ has one end a vertex of $C\left(G_{1}, S_{2}, q_{j}\right)$.

For each vertex $x$ of $G \cdot S_{2}$ we define $R(x)$ as the subgraph of $G$ formed by taking the union of $C\left(G_{1}\right.$, $\left.S_{2}, x\right)$ and those graphs $F_{j}$ for which $q_{j}=x$, and then adjoining the members of the corresponding sets $Z_{j}$ as new edges.

For a given $x$ the graph $R(x)$ may have only the one vertex $x$. If this is not so the set $E(R(x))$ is non-null and its vertices of attachment in $G$ are $x$ and $v_{1}$, since $x$ is not a cut-vertex of $G$.

Let us reverse all the non-null sets $R(x)$ (which are disjoint). By $9.24 G$ is then transformed into another graph $G^{\prime}$ with the same bond-matroid $M$. We note that $G^{\prime}$ has no isolated vertex. But the transformation replaces $H_{1}$ by an end-graph $K$ of $Y$ in $G^{\prime}$ such that $E(K)=E\left(H_{2}\right)-S_{2}$. But this is contrary to the choice of $G, H_{1}$, and $H_{2}$.

We deduce that in fact $\left|E\left(H_{2}\right)\right|=0$. The theorem follows.

\subsection{Property of Odd Matroids}

In this section we prove the following theorem. 9.31 Every odd binary matroid has a minor of Type BII, KI or KII.

Proof: Assume that the theorem fails. Then there exists an odd binary matroid $M$, on a set $E$, which has no minor of Type BII, KI, or KII. Choose such a matroid so that $|E|$ has the least possible value.
By definition there is at least one atom $Y$ of $M$ which is not bridge-separable.

There must be a cyclic sequence

$$
P=\left(B_{1}, B_{2}, \ldots, B_{2 n+1}, B_{1}\right)
$$

of an odd number $2 n+1$, where $n \geqslant 1$, of distinct bridges $B_{i}$ of $Y$ in $M$ such that each overlaps its two neighbors in the sequence. We refer to $P$ as an odd overlap-circuit of $Y$.

We observe here another intrusion of graph theory into the general theory of matroids. The bridges $B_{i}$ can be regarded as the vertices of a graph whose edges are the overlapping pairs. We have used the well-known theorem that a graph is bipartite if and only if it has no polygon with an odd number of edges.

We proceed by a sequence of subsidiary theorems. 9.311 Let $Y$ be any atom of $M$ which is not bridgeseparable. Then the bridges of $Y$ in $M$ can be arranged in a single odd overlap-circuit $P$ so that two bridges overlap only if they are consecutive in $P$.

Proof: Choose an odd overlap-circuit $P$ of $Y$ having as few terms as possible. This clearly implies that nonconsecutive members of $P$ do not overlap.

Let $S$ be the union of the members of $P$. Then $P$ is an odd overlap-circuit of $M \times(S \cup Y)$, by 8.53 . But every minor of $M \times(S \cup Y)$ is a minor of $M$. Hence $E=S \cup Y$, by the choice of $M$. The theorem follows.

In the next few propositions we suppose given an atom $Y$ of $M$, with a corresponding odd overlap-circuit

$$
P=\left(B_{1}, B_{2}, \ldots, B_{2 n+1}, B_{1}\right) .
$$

9.312 Suppose there is an atom $Z$ of $M \cdot(E-Y)$ on $B_{i}$ which is skew to both $B_{i-1}$ and $B_{i+1}$. Then $B_{i}$ consists of a single cell.

Proof: With regard to the suffices we adopt the convention that $B_{0}=B_{2 n+1}, B_{-1}=B_{2 n}$, and in general $B_{r}=B_{2 n+1+r}$.

Write $S=E-\left(B_{i}-Z\right)$. The bridges $B_{j}$ of $Y$ other than $B_{i}$ are also bridges of $Y$ in $M \times S$, determining the same partitions of $Y$ as in $M$, by 8.53 .

On the other hand $Z$ is an atom of $((M \cdot(E-Y))$ $\left.\times B_{i}\right) \times Z$, that is of $\left(M \cdot B_{i}\right) \times Z$ by 3.332 and 3.42 . But this matroid is $(M \times S) \cdot Z=((M \times S) \cdot(S-Y)) \cdot Z$. Hence $Z$ is the only remaining bridge of $Y$ in $M \times S$.

Since $Y \cup Z$ is a line of $M$ on $M \times S$ the atom $Z$ determines the same partition $\{U, V\}$ of $Y$ in $M \times S$ as in $M$. Each of the sets $U$ and $V$ meets at least two members of $\pi\left(M, B_{i-1}, Y\right)$ and at least two of $\pi\left(M, B_{i+1}\right.$, $Y)$.

From the above results we deduce that the replacement of $B_{i}$ by $Z$ in $P$ yields an odd overlap-circuit of $M \times S$. Hence $B_{i}$ is monatomic, with the single atom $Z$, by the choice of $M$.

Now let $a$ be any cell of $Z$. Write $T=E-(Z-\{a\})$. We have $Y \epsilon M \cdot T$, by 8.531 . The bridges of $Y$ in $M$, other than $Z$, are also bridges of $Y$ in $M \cdot T$, determining the same partitions of $Y$ as in $M$, by 8.531 . The only other bridge of $Y$ in $M \cdot T$ is the atom $\{a\}$.

$Z$ determines the partition $\{U, \mathrm{~V}\}$ of $Y$ in $M$. There exists $X^{\prime} \in M \cdot T$ such that $\{a\} \subseteq X^{\prime} \subseteq Z \cup U \epsilon M$. There 
exists $X \epsilon M$ such that $X \cap T=X^{\prime}$. But this implies $X \subseteq Z \cup U$, and therefore $X=Z \cup U$, by Axiom $\mathrm{I}$. Hence $X^{\prime}=U \cup\{a\}$. Accordingly $a$ determines the same partition of $Y$ in $M \cdot T$ as does $Z$ in $M$.

We deduce that the replacement of $B_{i}=Z$ by $\{a\}$ in $P$ yields an odd overlap-circuit of $M \cdot T$. Hence $B_{i}=\{a\}$, by the choice of $M$.

We refer to a bridge consisting of a single cell as unicellular.

9.313 If $n \geqslant 2$, then every bridge of $Y$ in $M$ is unicellular

Proof: Consider any bridge $B_{i}$ in $P$. Now $B_{i}$ and $B_{i+1}$ are not equipartite 3-bridges, for otherwise $B_{i-1}$ and $B_{i+1}$ would overlap, contrary to 9.311 . Hence $B_{i}$ is skew to $B_{i+1}$, and similarly to $B_{i-1}$, by 8.44 . But $B_{i-1}$ and $B_{i+1}$ are not skew, by 9.311 . Hence there is an atom $Z$ of $M \cdot(E-Y)$ on $B_{i}$ which is skew to both $B_{i-1}$ and $B_{i+1}$, by 8.42. Accordingly $B_{i}$ is unicellular, by 9.312 .

Let $\Sigma$ be the class of all non-null subsets of $Y$ of the form

$$
\prod_{i=1}^{2 n=1} W_{i},
$$

where $W_{i} \in \pi\left(M, B_{i}, Y\right)$. Clearly the members of $\Sigma$ are disjoint and their union is $Y$.

9.314 If $Q \in \Sigma$, then $|Q|=1$. Moreover there is an integer $i$ such that $Y-Q=W_{i} \cup W_{i+1}$, where $W_{i} \epsilon \pi(M$, $\left.B_{i}, Y\right)$ and $W_{i+1} \in \pi\left(M, B_{i+1}, Y\right)$.

Proof: Choose $a \epsilon Q$. Now $Y-\{a\} \epsilon M \cdot(E-\{a\})$, by 8.54. But $P$ is not an odd overlap-circuit of $Y-\{a\}$ in $M \cdot(E-\{a\})$, by the choice of $M$. It follows from 8.54 that $Q-\{a\}$ must be null. Moreover there is an integer $i$ such that $B_{i}$ and $B_{i+1}$ do not overlap as bridges of $Y-\{a\}$ in $M \cdot(E-\{a\})$. The proposition follows.

Suppose $n \geqslant 2$. Then each bridge of $Y$ in $M$ is unicellular, by 9.313. Write $B_{i}=\left\{b_{i}\right\}$. For each $i$ the atom $\left\{b_{i}\right\}$ of $M \cdot(E-Y)$ determines a partition

$$
\pi\left(M, B_{i}, Y\right)=\left\{S_{i}, T_{i}\right\}
$$

of $Y$. Since consecutive members of $P$ overlap neither $S_{i}$ nor $T_{i}$ is null.

Consider a particular bridge $B_{i}$. By 9.311 we can adjust the notation so that $S_{j} \subset S_{i}$ or $S_{j} \subset T_{i}$ whenever $B_{j}$ is distinct from and not consecutive with $B_{i}$ in $P$. We choose one $B_{j}$ of this kind and arrange, by interchanging $S_{i}$ and $T_{i}$ if necessary, that $S_{j} \subset T_{i}$.

Let $B_{k}$ be the first member of the sequence

$$
\left(B_{j}, B_{j+1}, \ldots, B_{i-2}\right)
$$

such that $S_{k+1} \cap S_{i}$ is non-null. Then $S_{k+1}$ meets both $S_{i}$ and $T_{i}$, since $B_{k+1}$ is skew to $B_{k}$. Hence $B_{k+1}$ is consecutive with $B_{i}$ in $P$, that is $B_{k}=B_{i-2}$.

Similarly if $B_{l}$ is the last member of the sequence

$$
\left(B_{i+2}, B_{i+3}, \ldots, B_{j}\right)
$$

such that $S_{l-1} \cap S_{i}$ is non-null we find that $l=i+2$.

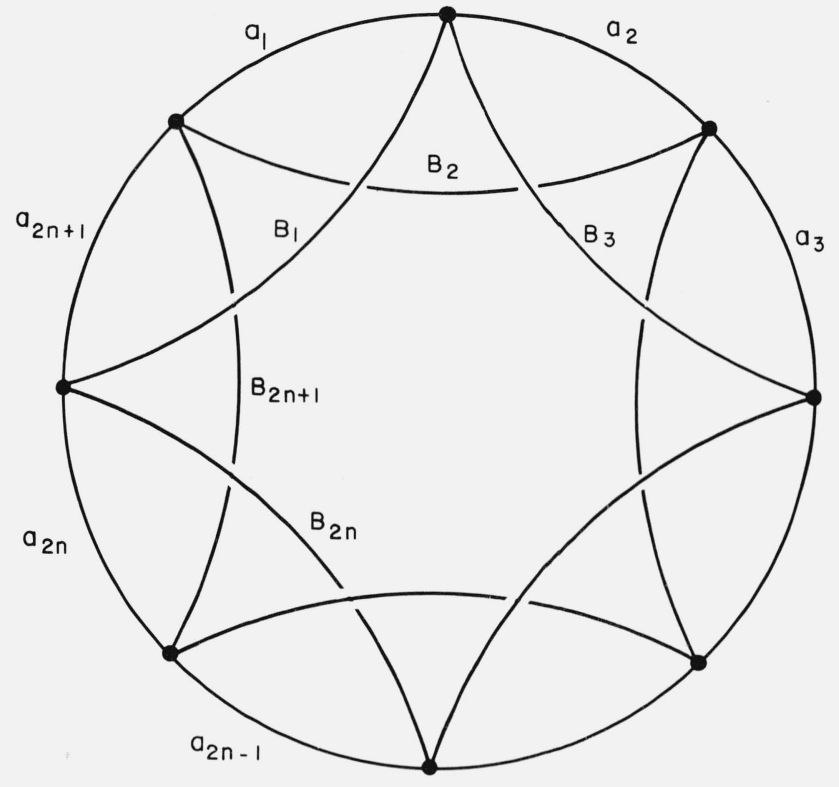

Figure 9G

It follows that $S_{h} \subset T_{i}$ for each $B_{h}$ not consecutive with $B_{i}$ in $P . \quad(h \neq i$.

By the result just proved we can adjust the notation so that $S_{i}$ contains neither $S_{j}$ nor $T_{j}$ as a subset whenever $B_{i}$ and $B_{j}$ are distinct. Then by 9.311 we find that $S_{i} \cap S_{j}$ is non-null if and only if $B_{i}$ and $B_{j}$ are consecutive in $P$.

It follows that the $2 n+1$ sets $S_{i} \cap S_{i+1},(1 \leqslant i \leqslant 2 n+1)$ are distinct members of $\Sigma$. We must now show that they are the only members of $\Sigma$.

Choose any $W \epsilon \Sigma$. By 9.314 we can write $W=S_{i}$ $\cap S_{i+1}, S_{i} \cap T_{i+1}, T_{i} \cap S_{i+1}$ or $T_{i} \cap T_{i+1}$, for some $i$. In the first case there is nothing to prove. In the second case we observe that $S_{i-1}$ meets $S_{i}$ and is a subset of $T_{i+1}$. Hence $W$ is the member $S_{i-1} \cap S_{i}$ of $\Sigma$. We deal with the third case similarly, using $S_{i+2}$ instead of $S_{i-1}$. In the fourth case we have $S_{i+3} \subseteq T_{i} \cap T_{i+1}=W$, whence $S_{i+3}=W$. But then $B_{i+3}$ overlaps no other bridge of $Y$, since $T_{i+3} \cup W=Y$, and this is a contradiction.

We are now in a position to prove 9.315 The assumption that $n \geqslant 2$ leads to the contradiction that $M$ is a matroid of Type KI.

Proof: The above diagram represents $M$, on the as yet unproved assumption that it is cographic. We write $a_{i}$ for the single cell of the member $S_{i} \cap S_{i+1}$ of $W$. (See 9.314.)

If $n>2$ we write $Y^{\prime}=\left(Y-S_{1}\right) \cup B_{1}$. By $8.55 Y^{\prime} \epsilon M$ and $B_{3}, B_{4}, \ldots$., $B_{2 n}$ are bridges of $Y^{\prime}$ in $M$. Moreover there is another bridge $B^{\prime}$ which contains $S_{1}, B_{2}$, and $B_{2 n+1}$. It also follows from 8.55 that $\left(B^{\prime}, B_{3}, B_{4}, \ldots\right.$., $B_{2 n}, B^{\prime}$ ) is an odd overlap-circuit of $Y^{\prime}$ in $M$. This contradicts 9.312 since $B^{\prime}$ is not unicellular. 


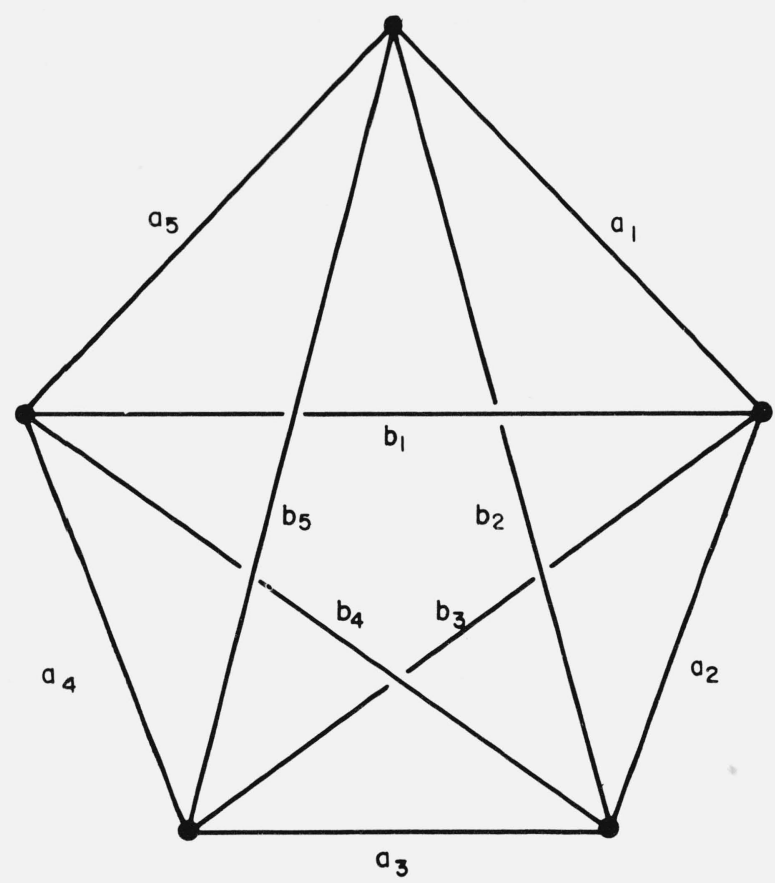

FigURE 9H

In the remaining case $n=2$. (Fig. 9H.)

Let us write $a_{i}=L_{i, i+1}$ if $1 \leqslant i \leqslant 4$, and $a_{5}=L_{1,5}$. We put also $b_{i}=L_{i, i+2}$ if $1 \leqslant i \leqslant 3, b_{4}=L_{1,4}$, and $b_{5}=L_{2,5}$. We construct a graph $G$ such that $E(G)=E$. We take it to have just five vertices $v_{1}, v_{2}, v_{3}, v_{4}$, and $v_{5}$, the ends of $L_{i, j}$ in $G$ being $v_{i}$ and $v_{j}$. Then $G$ is a complete 5-graph.

We find that $M=P(G)$. To prove this we observe that $Y$ and the five sets $\left\{b_{i}\right\} \cup S_{i}$ are atoms of both $M$ and $P(G)$. Any atom $X$ of $M$ is a linear combination of these six. For otherwise some combination of atoms of $M$ would be a non-null proper subset of $Y$, which, by 5.34 , is contrary to Axiom $\mathrm{I}$. Hence $X$ is a mod 2 sum of atoms of $P(G)$ and so has an atom $X^{\prime}$ of $P(G)$ as a subset, by 5.34. Similarly any atom of $P(G)$ contains an atom of $M$.

We deduce that $M=P(G)$, by Axiom I. Thus $M$ is of Type KI.

We may now assume that no atom of $M$ has an odd overlap-circuit constituted by more than three bridges.

We select a particular atom $Y$ which is not bridgeseparable, and we denote its three mutually overlaping bridges by $B_{1}, B_{2}$, and $B_{3}$.

In our analysis we make use of the following proposition.

9.316 Suppose $W_{1}$ is a member of $\pi\left(M, B_{1}, Y\right)$ with the property that some atom $Z$ of $M \cdot(E-Y)$ on $B_{1}$ determines the partition $\left\{W_{1}, Y-W_{1}\right\}$ of $Y$. Then either $\pi\left(M, B_{2}, Y\right)$ or $\pi\left(M, B_{3}, Y\right)$ has a member which cuts $W_{1}$.

Proof: Assume the contrary. Then there exist $W_{2} \in \pi\left(M, B_{2}, Y\right)$ and $W_{3} \in \pi\left(M, B_{3}, Y\right)$ such that $W_{1} \subseteq W_{2}$ $\cap W_{3}$.
Let $Y^{\prime}$ denote the atom $\left(Y-W_{1}\right) \cup Z$ of $M$. It follows from 8.55 that $B_{2}$ and $B_{3}$ are overlapping bridges of $Y^{\prime}$ in $M$.

Now $W_{1}$ is an atom of $M \cdot\left(E-Y^{\prime}\right)$, by 8.55. Suppose it meets another atom $X$ of $M \cdot\left(E-Y^{\prime}\right)$. Since $X-W_{1}$ is non-null, and $X$ cannot be an atom of the bridge $B_{2}$ or $B_{3}$ of $Y^{\prime}$ we have $X \subseteq B_{1} \cup Y^{\prime} \subset B_{1} \cup Y$. There is thus an atom of $M \times\left(B_{1} \cup Y\right)$ which cuts $W_{1}$. This is impossible, by 8.51 and 8.52 . We deduce that $W_{1}$ is another bridge of $Y^{\prime}$ in $M$. Determining the partition $\left\{Y-W_{1}, Z\right\}$ of $Y^{\prime}$ it overlaps neither $B_{2}$ nor $B_{3}$.

Let $H$ be the class of all remaining bridges of $Y^{\prime}$ in $M$. The union of the members of $H$ is $B_{1}-Z$. This set is non-null since $Z$ determines the partition $\left\{Y-W_{1}, W_{1}\right\}$ of $Y$, while $B_{1}$ overlaps $B_{2}$ and $B_{3}$.

Applying 8.55 with $Y$ and $Y^{\prime}$ interchanged we see that if $B \epsilon H$, then no member of $\pi\left(M, B, Y^{\prime}\right)$ can contain $Z$.

Since $B_{1}$ and $B_{2}$ overlap with respect to $Y$ there is an atom $X$ of $M$ on $B_{1} \cup Y-W_{1}=B_{1} \cup Y^{\prime}$ which cuts $Y-W_{2}$. Then $X \cap\left(E-Y^{\prime}\right)$ is a union of disjoint atoms of $M \cdot\left(E-Y^{\prime}\right)$, all contained in $B_{1} \cup Y^{\prime}$, by 8.51. Let us enumerate them as $X_{1}, X_{2}, \ldots, X_{k}$. Let $X_{i}$ determine the partition $\left\{S_{i}, T_{i}\right\}$ of $Y^{\prime}$. The $\bmod 2$ sum of $X$ and the atoms $X_{i} \cup S_{i}$ of $M$ is contained in $Y^{\prime}$, and is either $Y^{\prime}$ or $\phi$. by 5.32 and Axiom I. Hence some $X_{i}$ cuts $Y-W_{2}$. This $X_{i}$ must be on some member of $H$.

We deduce that there exists $C_{1} \epsilon H$ such that no member of $\pi\left(M, C_{1}, Y^{\prime}\right)$ contains either $Z$ or $Y-W_{2}$. Then $C_{1}$ overlaps both $W_{1}$ and $B_{2}$ as bridges of $Y^{\prime}$. Similarly there exists $C_{2} \epsilon H$ overlapping both $W_{1}$ and $B_{3}$.

We may have $C_{1}=C_{2}$. In that case $\left(B_{2}, B_{3}, C_{1}\right)$ is an odd overlap-circuit of $Y^{\prime}$ which does not include $W_{1}$, and this contradicts 9.311 .

In the remaining case $Y^{\prime}$ has an odd overlap-circuit $\left(B_{2}, B_{3}, C_{2}, W_{1}, C_{1}, B_{2}\right)$ having five members. But such odd overlap-circuits are ruled out by 9.315 .

The proposition follows.

By 8.43 and $9.316 B_{1}, B_{2}$, and $B_{3}$ are not equipartite 3-bridges.

Suppose $\pi\left(M, B_{1}, Y\right)=\pi\left(M, B_{2}, Y\right)=\left\{W_{1}, W_{2}, W_{3}\right\}$. Then $B_{3}$ is skew to $B_{1}$ and $B_{2}$, by 8.44 . But $B_{1}$ and $B_{2}$ are clearly not skew to each other. Hence $B_{3}$ is unicellular, by 9.312. It determines a partition $\{S, T\}$ of $Y$.

We may suppose without loss of generality that $W_{1} \cap S$ is non-null. Then $W_{1} \cap S \in \Sigma$. Moreover $W_{1} \cap S$ is a proper subset of $W_{1}$, by 8.43 and 9.316 . In view of 9.314 we may write, without loss of generality,

$$
Y-\left(W_{1} \cap S\right)=T \cup W_{i},
$$

where $i=1$ or 2 . In the apparent alternative $Y-\left(W_{1} \cap S\right)=S \cup W_{i}$ we have $Y=S \cup W_{i}$, which is impossible since $B_{1}$ and $B_{3}$ overlap. But then $W_{3} \subseteq T$, $W_{3} \in \Sigma$, and we have a contradiction of 8.43 and 9.316 .

We may now suppose that $B_{1}, B_{2}$, and $B_{3}$ are mutually skew, by 8.44. Applying 8.42 and 9.312 we find that two of them, say $B_{1}$ and $B_{2}$, are unicellular. 
Let them determine partitions $\left\{S_{1}, T_{1}\right\}$ and $\left\{S_{2}\right.$, $\left.T_{2}\right\}$ of $Y$ respectively. We write

$$
\pi\left(M, B_{3}, Y\right)=\left\{W_{1}, W_{2}, \ldots ., W_{k}\right\} .
$$

The number $k$ is at least 2 , since the three bridges overlap.

9.317 The assumption that $k=2$ leads to the contradiction that $M$ has a minor of Type BII or KII.

Proof: On this assumption $B_{3}$ must be unicellular, by 9.312 .

Suppose $W_{1}$ does not meet both $S_{1} \cap S_{2}$ and $T_{1} \cap T_{2}$. Then it meets both $S_{1} \cap T_{2}$ and $S_{2} \cap T_{1}$, for it is not contained in any of the sets $S_{1}, S_{2}, T_{1}, T_{2}$, by the overlapping of the bridges. We may therefore adjust the notation so that $W_{1}$ meets both $S_{1} \cap S_{2}$ and $T_{1} \cap T_{2}$.

Assume that $W_{1}$ contains neither $S_{1} \cap T_{2}$ not $S_{2} \cap T_{1}$. Then $M$ has a minor of Type BII, by 8.64, which is contrary to the choice of $M$. We may therefore make a further adjustment of notation so that $S_{1} \cap T_{2} \subseteq W_{1}$.

We now observe that $W_{1}$ cuts both $S_{1} \cap S_{2}$ and $T_{1} \cap T_{2}$. For otherwise $W_{2}$ would be a subset of $T_{1}$ or $S_{2}$, and $B_{3}$ would not overlap both $B_{1}$ and $B_{2}$. Another application of 8.64 shows that $S_{2} \cap T_{1} \subseteq W_{2}$.

We can now list the members of $\Sigma$, each unicellular by 9.314 , as follows.

$$
\begin{aligned}
& S_{1} \cap S_{2} \cap W_{1}=\left\{L_{1,1}\right\}, \\
& S_{1} \cap S_{2} \cap W_{2}=\left\{L_{2,1}\right\} \\
& S_{2} \cap T_{1} \cap W_{2}=\left\{L_{2,2}\right\} \\
& T_{1} \cap T_{2} \cap W_{2}=\left\{L_{3,2}\right\} \\
& T_{1} \cap T_{2} \cap W_{1}=\left\{L_{3,3}\right\} \\
& S_{1} \cap T_{2} \cap W_{1}=\left\{L_{1,3}\right\} .
\end{aligned}
$$

We also write $B_{1}=\left\{L_{2,3}\right\}, B_{2}=\left\{L_{1,2}\right\}$ and $B_{3}=\left\{L_{3,1}\right\}$. This situation is represented in figure $9 \mathrm{I}$, on the assumption that $M$ is cographic.

We now construct a graph $G$ with six vertices $a_{1}$, $a_{2}, a_{3}, b_{1}, b_{2}, b_{3}$, taking the cells $L_{i, j}$ as edges. The ends of $L_{i, j}$ are $a_{i}$ and $b_{j}$. Thus $G$ is a Thomsen graph. By an argument like the one which concludes the proof of 9.315 we find that $M=P(G)$.

Thus $M$ is a matroid of Type KII, contrary to the choice of $M$.

We may now suppose $k \geqslant 3$.

One of $S_{1} \cap S_{2}$ and $T_{1} \cap T_{2}$ must be contained in a member of $\pi\left(M, B_{3}, Y\right)$. For otherwise we could find an atom $Z$ of $\boldsymbol{M} \cdot(\boldsymbol{E}-Y)$ on $B_{3}$ cutting both $S_{1} \cap S_{2}$ and $T_{1} \cap T_{2}$, by 4.37 and 8.41 . Then $Z$ would be skew to both $B_{1}$ and $B_{2}$, and $B_{3}$ would be unicellular, by 9.312. This is impossible since $k \geqslant 3$. Similarly one of $S_{1} \cap T_{2}$ and $S_{2} \cap T_{1}$ is contained in a member of $\pi\left(M, B_{3}, Y\right)$.

We may thus adjust the notation so that

$$
S_{1} \cap S_{2} \subseteq W_{1}, \quad S_{1} \cap T_{2} \subseteq W_{2} .
$$

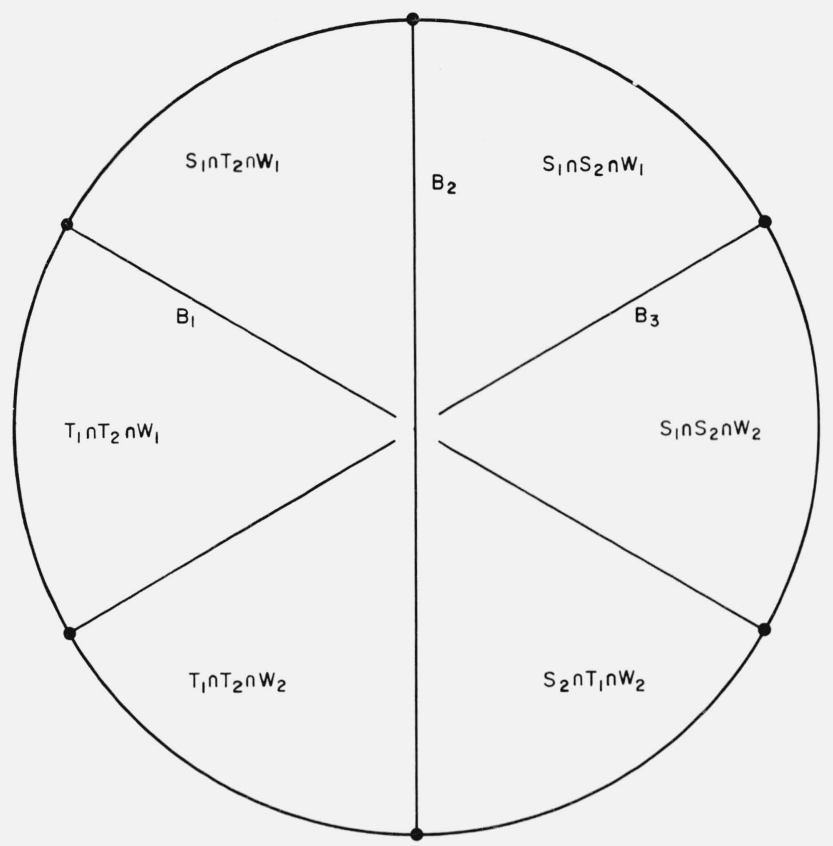

Figure 9I

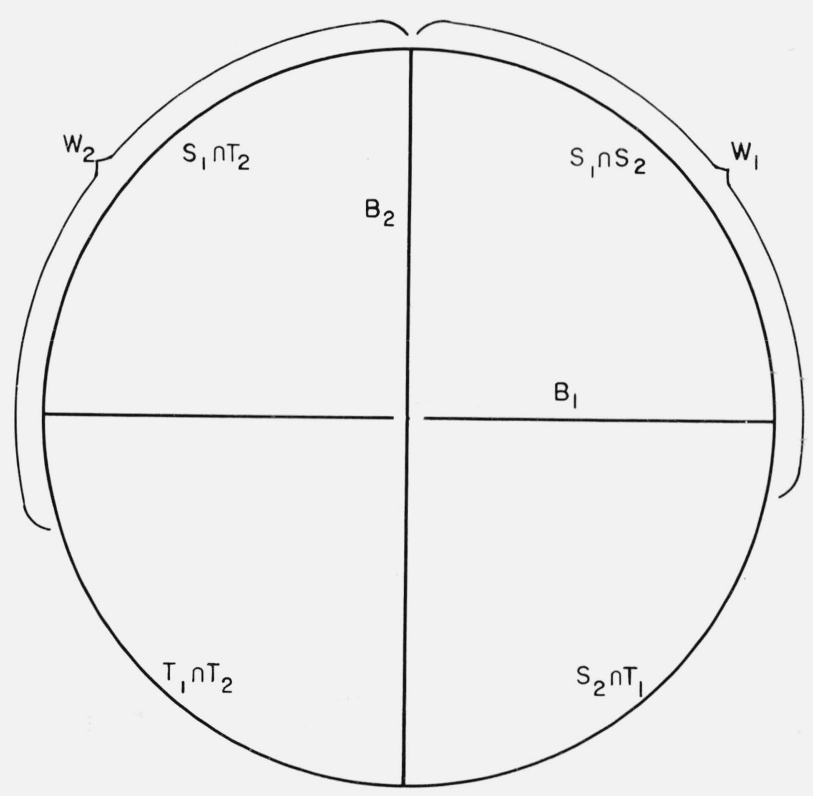

FiguRE 9J

For $S_{1} \cap S_{2}$ and $S_{1} \cap T_{2}$ are not both in $W_{1}$; otherwise $B_{1}$ and $B_{3}$ would not overlap.

We note that $W_{1}$ does not meet $T_{1} \cap T_{2}$. For otherwise $W_{1}$ would contain $S_{1} \cap T_{2}$ or $S_{2} \cap T_{1}$, by 8.64 , since $M$ has no minor of Type BII. But then $W_{1}$ would contain $S_{1}$ or $T_{2}$, and $B_{3}$ would not overlap both $B_{1}$ and $B_{2}$. Similarly $W_{2}$ does not meet $S_{2} \cap T_{1}$.

Suppose $W_{1}$ meets $S_{2} \cap T_{1}$. This case is represented schematically in figure 9J. 
By 9.314 we have

$$
Y-\left(W_{1} \cap S_{2} \cap T_{1}\right)=S_{1} \cup T_{2}, S_{1} \cup W_{x} \text { or } T_{2} \cup W_{x},
$$

for a suitable suffix $x$. The first alternative implies that $S_{2} \cap T_{1} \subseteq W_{1}$. But then $S_{2} \subseteq W_{1}$ which is impossible since $\bar{B}_{2}$ and $B_{3}$ overlap.

The second possibility requires $T_{1} \cap T_{2} \subseteq W_{x}$ and $W_{1} \cup W_{2} \cup W_{x}=Y$. Thus $x=k=3$. But now $W_{2}$ is a subset of $S_{1}$ and of $T_{2}$. Hence this case is ruled out by 8.43 and 9.316 .

In the third case $S_{1} \cap S_{2} \subseteq W_{x}$, so that $W_{x}=W_{1}$. But it is then necessary that $S_{2} \cap T_{1} \subseteq W_{1}$. This is impossible since it requires $S_{2} \subseteq W_{1}$.

We deduce that $W_{1}=S_{1} \cap S_{2}$. Similarly $W_{2}=S_{1} \cap T_{2}$.

Let $W_{3}$ be a member of $\pi\left(M, B_{3}, Y\right)$ which meets $S_{2} \cap T_{1}$. Then by 9.314 we have

$$
Y-\left(W_{3} \cap S_{2} \cap T_{1}\right)=S_{1} \cup T_{2}, S_{1} \cup W_{x} \text { or } T_{2} \cup W_{x} .
$$

The first alternative implies $S_{2} \cap T_{1} \subseteq W_{3}$, the second that $k=3$ or 4 and $T_{1} \cap T_{2} \subseteq W_{x}$. The third implies $S_{1} \cap S_{2} \subseteq W_{x}=W_{1}$. Then $S_{2} \cap T_{1} \subseteq W_{3}$.

Similar results are obtained when we consider a member of $\pi\left(M, B_{3}, Y\right)$ meeting $T_{1} \cap T_{2}$. Since $S_{2} \cap T_{1}$ and $T_{1} \cap T_{2}$ are not contained in the same member of $\pi\left(M, B_{3}, Y\right)$, by the overlapping of $B_{1}$ and $B_{3}$, it follows that we can adjust the notation so that

$$
\begin{aligned}
\pi\left(M, B_{3}, Y\right) & =\left\{W_{1}, W_{2}, W_{3}, W_{4}\right\}, \\
W_{1} & =S_{1} \cap S_{2}, \\
W_{2} & =S_{1} \cap T_{2}, \\
W_{3} & \subseteq S_{2} \cap T_{1}, \\
T_{1} \cap T_{2} & \subseteq W_{4} .
\end{aligned}
$$

Now there is an atom $Z$ of $M \cdot(E-Y)$ on $B_{3}$ which cuts both $W_{1} \cup W_{2}$ and $W_{1} \cup W_{3}$, by 4.37 and 8.41. This atom must determine one of the partitions $\left\{W_{1}, Y-W_{1}\right\}$ and $\left\{W_{1} \cup W_{4}, W_{2} \cup W_{3}\right\}$ of $Y$. The latter requires $M$ to have a minor of Type BII, by 8.64, and can be ruled out. But now we have a contradiction of 9.316, since $W_{1} \subseteq S_{1}$ and $W_{1} \subseteq S_{2}$.

The analysis is now complete. No matroid satsifies the conditions imposed on $M$, and theorem 9.31 is established.

\subsection{Characterization of Graphic Matroids}

One more major result is required to complete our theory.

9.41 If a matroid $M$ is regular and has no minor of Type KI or KII, then $M$ is graphic.

Proof: Assume the contrary. Then there is a regular matroid $M$ on a set $E$ which has no minor of Type KI or KII but which is not graphic. Choose $M$ so the $d M$ has the least possible value.
Suppose first that $M$ is not connected. We can find complementary non-null separators $S_{1}$ and $S_{2}$ of $M$. There exist graphs $G_{1}$ and $G_{2}$ such that

$$
B\left(G_{1}\right)=M \times S_{1}, B\left(G_{2}\right)=M \times S_{2},
$$

by 5.62. We may take $V\left(G_{1}\right)$ and $V\left(G_{2}\right)$ to be disjoint subsets of some larger set $V$. Then $G_{1}$ and $G_{2}$ together constitute a graph $G$. We note that $S_{1}$ and $S_{2}$ are separators of $B(G)$, by 9.14 .

If $X \epsilon B(G)$ we have

$$
X \epsilon B(G) \times S_{i}=B(G) \cdot S_{i}=B\left(G \cdot S_{i}\right)=B\left(G_{i}\right)=M \times S_{i},
$$

where $i=1$ or 2 . Hence $X \epsilon M$. We find similarly that if $X \epsilon M$ then $X \epsilon B(G)$. We deduce that $M=B(G)$, a contradiction.

We may now suppose $M$ connected.

Suppose $d M=-1$. Then $|E|=0$ or 1 since $M$ is connected. We thus have $M=B(G)$ for some graph $G$ with just one vertex. If $|E|=0, G$ is edgeless. If $|E|=1 G$ has a single loop.

If $d M=0$ we have $M=\{E\}$, by 3.57 and the connection of $M$. Then $M=B(G)$, where $G$ has just two vertices and each cell of $M$ is an edge incident with each of them.

Next suppose $d M=1$. Then $E$ is a connected line of $M$. Let its three points be $X_{1}, X_{2}$, and $X_{3}$. Each is the mod 2 sum of the others, by 7.12. Hence they have no common cell. The sets $X_{1} \cap X_{2}, X_{2} \cap X_{3}$, and $X_{3} \cap X_{1}$ are non-null, by Axiom $\mathrm{I}$, and their union is $E$. Let $G$ be a graph with just three vertices $v_{1}, v_{2}$, and $v_{3}$, and such that $E(G)=E$. We take each member of $X_{i} \cap X_{j}$ to have ends $v_{i}$ and $v_{j},(1 \leqslant i<j \leqslant 3)$. It is readily verified that $M=B(G)$.

We deduce that $d M \geqslant 2$.

Now $M$ has no minor of Type BI, BII, KI, or KII, by 7.51. Hence there is an atom $Y$ of $M$ having two distinct bridges, by 8.63. Moreover $M$ is even, by 9.31, and therefore $Y$ is bridge-separable. We can arrange the bridges of $Y$ in $M$ in two disjoint classes $P$ and $Q$ so that no two members of the same class overlap, and we can clearly arrange that $P$ and $Q$ are non-null.

Let $S$ and $T$ be the unions of the members of $P$ and $Q$ respectively. The matroids $M \times(S \cup Y)$ and $M \times(T \cup Y)$ are connected, by 3.48 and 8.13 . For if the $S$ of 8.13 is a bridge of $Y$ the condition $(M \cdot(E-Y)) \times S=M \times S$ is equivalent to $M \cdot S=M \times S$. (See 3.42.)

There exist graphs $H$ and $K$ such that

$$
\begin{aligned}
& B(H)=M \times(S \cup Y), \\
& B(K)=M \times(T \cup Y),
\end{aligned}
$$

by the choice of $M$.

Hence we may choose $H$ to have a vertex $h$ which is incident with the members of $Y$ and no other edges, by 9.25 and 8.53. Similarly $K$ may be supposed to have a vertex $k$ incident only with the members of $Y$. 
We may take $V(H)$ and $V(K)$ to be disjoint subsets of some larger set. We construct a graph $G$ as follows

$$
\begin{aligned}
& E(G)=E \\
& V(G)=(V(H)-\{h\}) \cup(V(K)-\{k\}) .
\end{aligned}
$$

If $A \epsilon E-Y$ the ends of $A$ in $G$ are its ends in $H$ or $K$. But if $A \epsilon Y$ then its ends in $G$ are its end other than $h$ in $H$ and its end other than $k$ in $K$. We illustrate this construction in figure $9 \mathrm{~K}$.

There is a binary chain-group $N$ such that $M=M(N)$.

Let $f$ be any chain of $N$. Its restriction to $E-Y$ is a sum of elementary chains $f_{1}, f_{2}, \ldots, f_{s}$ of $N \cdot(E-Y)$, by 5.32. Each $f_{i}$ has a domain restricted to one of the bridges in $Y$ in $M$. Hence $f_{i}$ is the restriction to $E-Y$ of a chain $g_{i}$ of $N$ such that $\left\|g_{i}\right\|$ is contained in $S \cup Y$ or $T \cup Y$. The sum of $f$ and the chains $g_{i}$ has a domain contained in $Y$. It is thus either zero or the elementary chain of $N$ with domain $Y$.

We conclude that $f$ is the sum of two chains $p$ and $q$ of $N$ with domains contained in $S \cup Y$ and $T \cup Y$ respectively.

The restriction of $p$ to $S \cup Y$ is a coboundary of a 0 -chain $l$ of $H$ over $G F(2)$. We may change each coefficient in $l$ by $l$ without altering $\delta l$, by the definition of a coboundary. We may therefore suppose $l(h)=0$. But then $p$ is the coboundary of a corresponding 0-chain in $G$. Similarly $q$ is a coboundary in $G$. Hence $f$ is such a coboundary.

Conversely suppose $f$ is a coboundary of a 0 -chain $l$ in $G$. Let $l_{1}$ and $l_{2}$ be formed from $l$ by replacing the coefficients of the vertices in $V(H)-\{h\}$ and $V(K)-\{k\}$ respectively by zeroes. Then $\delta l=\delta l_{1}+\delta l_{2}$. Moreover $\left\|\delta l_{1}\right\| \subseteq S \cup Y$ and $\left\|l_{2}\right\| \subseteq T \cup Y$. The restrictions to $S \cup Y$ and $T \cup Y$ of $\delta l_{1}$ and $\delta l_{2}$ are chains of $N \times(S \cup Y)$ and $N \times(T \cup Y)$ respectively. Hence $\delta l_{1}$ and $\delta l_{2}$ are chains of $N$, and therefore $\delta l$ is a chain of $N$.

We deduce that $N$ is the coboundary group of $G$. Hence $M=M(N)=B(G)$, contrary to the choice of $M$. The theorem follows.

We may now combine some of the preceding results into the following theorem.
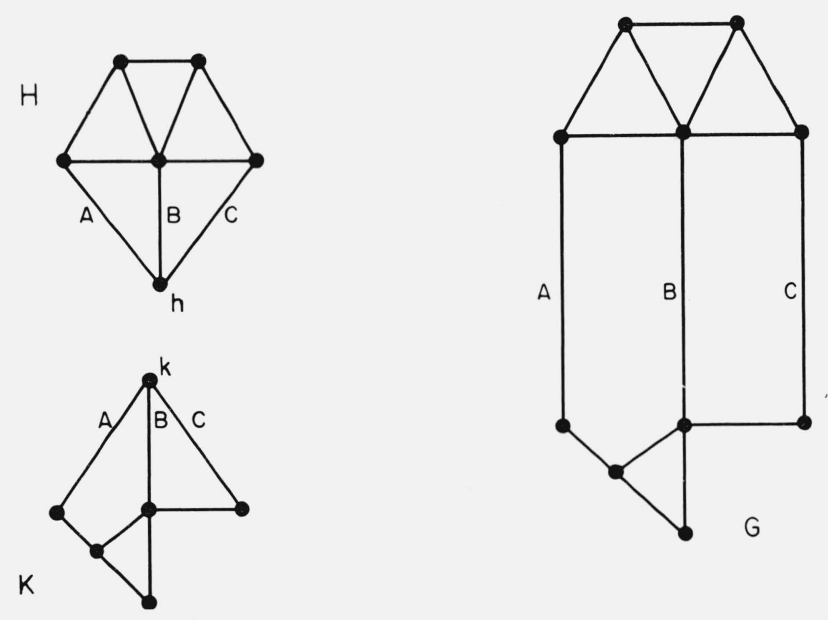

FigURE 9K

$9.42 \mathrm{~A}$ binary matroid is graphic if and only if it is regular and has no minor of Type KI or KII.

Proof: If $M$ satisfies this condition it is graphic, by 9.41 . Conversely if $M$ is graphic it is regular, by 2.54. No minor of $M$ is of Type KI or KII, by 5.62, for matroids of these two types are odd, by 8.31 , and therefore not graphic, by 9.23 .

Cographic matroids can be characterized by a dual form of 9.42. They are those regular matroids for which no minor is the bond-matroid of a Kuratowski graph.

A matroid is planar if it is both graphic and cographic. It is possible to derive Kuratowski's Theorem on planar graphs by applying 9.42 to the polygonmatroid of a planar graph.

An algorithm for determining whether a given binary matroid, given by a representative matrix, is graphic has recently been presented in the Canadian Journal of Mathematics 16, 108-127 (1964).

(Paper 69B1-131) 